\title{
Super-Ehlers in any dimension
}

\section{Sergio Ferrara, ${ }^{a, b, c}$ Alessio Marrani ${ }^{a}$ and Mario Trigiante $^{d}$}

${ }^{a}$ Physics Department, Theory Unit, CERN,

CH 1211, Geneva 23, Switzerland

${ }^{b}$ INFN - Laboratori Nazionali di Frascati,

Via Enrico Fermi 40, I-00044 Frascati, Italy

${ }^{c}$ Department of Physics and Astronomy, University of California, Los Angeles, CA U.S.A.

${ }^{d}$ Laboratory of Theoretical Physics, Dipartimento di Scienze Applicate e Tecnologia, Politecnico di Torino, C.so Duca degli Abruzzi, I-10129 Torino, Italy

E-mail: sergio.ferrara@cern.ch, alessio.marrani@cern.ch,

mario.trigiante@polito.it

AbStRact: We classify the enhanced helicity symmetry of the Ehlers group to extended supergravity theories in any dimension. The vanishing character of the pseudo-Riemannian cosets occurring in this analysis is explained in terms of Poincaré duality .The latter resides in the nature of regularly embedded quotient subgroups which are noncompact rank preserving.

KEYwords: Supergravity Models, Extended Supersymmetry

ARXIV EPRINT: 1206.1255 


\section{Contents}

1 Introduction 1

2 Clifford algebras and "pure" theories 4

$2.1 \quad N=16$ (maximal) 5

$\begin{array}{lll}2.2 N=12 & 12\end{array}$

$2.3 \quad N=10 \quad 12$

$3 \quad N=8,6$ matter coupled theories $\quad 12$

$\begin{array}{lll}3.1 N=8 & 12\end{array}$

$\begin{array}{lll}3.2 N=6 & 14\end{array}$

$4 \quad N=4$ matter coupled symmetric theories $\quad 14$

4.1 Minimal coupling infinite sequence and "pure" $D=4$ supergravity 17

4.2 "Pure" $D=5,6$ supergravity and $T^{3}$ and $S T^{2}$ models in $D=4 \quad 17$

$\begin{array}{lll}4.2 .1 \quad D=5 & 17\end{array}$

$\begin{array}{lll}4.2 .2 D=6 & 18\end{array}$

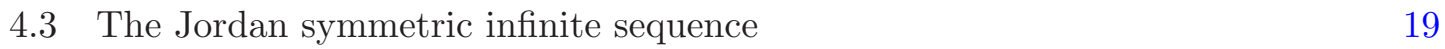

$\begin{array}{llr}\text { 4.3.1 } D=6 & 19\end{array}$

$\begin{array}{lll}4.3 .2 D=5 & 19\end{array}$

$\begin{array}{lll}\text { 4.3.3 } D=4 & 20\end{array}$

4.4 Magical models 20

$\begin{array}{lll}4.4 .1 & D=4 & 20\end{array}$

$\begin{array}{lll}4.4 .2 D=5 & 21\end{array}$

$\begin{array}{lll}\text { 4.4.3 } D=6 & 21\end{array}$

5 Cosets with $\chi=0$ and Poincaré duality 22

5.1 The cosets 26

$\begin{array}{lll}5.1 .1 N=16 & 26\end{array}$

$\begin{array}{lll}5.1 .2 N=12 & 26\end{array}$

$\begin{array}{lll}5.1 .3 N=10 & 28\end{array}$

$\begin{array}{lll}5.1 .4 N=8 & 28\end{array}$

$\begin{array}{lll}5.1 .5 N=6 & 28\end{array}$

$\begin{array}{lll}5.1 .6 N=4 \text { symmetric } & 28\end{array}$

5.2 Poincaré duality 32

$\begin{array}{lll}5.2 .1 \quad N=16 & 32\end{array}$

$\begin{array}{lll}\text { 5.2.2 } N=4 \text { Magical models } & 39\end{array}$

$\begin{array}{lll}5.3 & \text { Hodge involution and coset cohomology } & 42\end{array}$

6 Conclusion 46 
A.1 The embeddings $\mathrm{SL}(D-2, \mathbb{R}) \times \mathrm{E}_{11-D(11-D)} \subset \mathrm{E}_{8(8)}$

A.2 Other embeddings $\quad 50$

A.3 General features 53

B $\mathfrak{s o}(8,8)$ outer automorphisms and dual subalgebras of $\mathfrak{e}_{8(8)} \quad 54$

$\begin{array}{ll}\text { C Poincaré duality and level decomposition } & 58\end{array}$

\section{Introduction}

Three decades ago it was shown [1] that the $D$-dimensional Ehlers group $\operatorname{SL}(D-2, \mathbb{R})$ is a symmetry of $D$-dimensional Einstein gravity, provided that the theory is formulated in the light-cone gauge. For any $D \geqslant 4$-dimensional Lorentzian space-time, this results enables to identify the graviton degrees of freedom with the Riemannian coset

$$
\mathcal{M}_{\text {grav }}=\frac{S L(D-2, \mathbb{R})}{S O(D-2)},
$$

even if the action of the theory is not simply the sigma model action on this coset (with the exception of a theory reduced to $D=3$ ). In $D=4$, this statement reduces to the well known fact that the massless graviton described by the Einstein-Hilbert action with two degrees of freedom of \pm 2 helicity has an enhanced symmetry $\mathrm{SO}(2) \rightarrow \mathrm{SL}(2, \mathbb{R})$.

In $\mathcal{N}$-extended supergravity in $D$ dimensions, $U$-duality ${ }^{1}$ symmetries play an important role to uncover, in terms of geometrical constructions, the non-linear structure of the theories, whose most symmetric one is the theory with maximal supersymmetry $(2 N=32$ supersymmetries). Furthermore, $U$-duality symmetries get unified with the Ehlers spacetime symmetry if one descends to $D=3[5,6]$. In the maximal case, the $D=3 U$-duality group is $E_{8(8)}$, with maximal compact subgroup (mcs) $\mathrm{SO}(16)$, which is also the underlying Clifford algebra for massless supermultiplets of maximal supersymmetry. As a consequence, the bosonic sector of the theory is described by the sigma model $E_{8(8)} / \mathrm{SO}(16)$ [7-9].

Following these preliminaries, it comes as no surprise that it was further discovered that in light-cone Hamiltonian formulation maximal supergravity exhibits $E_{7(7)}$ symmetry in $D=4[11]$ and $E_{8(8)}$ symmetry in $D=3$ [12] (for the $D=11$ theory, see [10]). Indeed, in any space-time dimension $D$ and for any number of supersymmetries $\mathcal{N}=2 N$, it is known that the $D=3 U$-duality group $G_{N}^{3}$ [13] embeds (through a rank-preserving embedding; for some basic definitions, see the start of appendix A) the Ehlers group $\operatorname{SL}(D-2, \mathbb{R})$ as a commutant of the $U$-duality group $G_{N}^{D}[16,17]$ :

$$
G_{N}^{3} \supset G_{N}^{D} \times \operatorname{SL}(D-2, \mathbb{R}) .
$$

\footnotetext{
${ }^{1}$ Here $U$-duality is referred to as the "continuous" symmetries of $[2,3]$. Their discrete versions are the $U$-duality non-perturbative string theory symmetries introduced by Hull and Townsend [4].
} 
It is then natural to conjecture that in a suitable light-cone formulation of any $\mathcal{N}$ extended supergravity theories $G_{N}^{D} \times \mathrm{SL}(D-2, \mathbb{R}$ ) (which we dub super-Ehlers group) is a manifest symmetry of the theory. Even if the super-Ehlers group is a bosonic extension of the Ehlers group itself, the presence of the $U$-duality commutant $G_{N}^{D}$ in (1.2) is closely related to supersymmetry. It is intriguing to notice that the super-Ehlers symmetries, which we classify below in any dimension, sometimes exhibit an "enhancement" into some larger group ${ }^{2}$ this occurs whenever the embedding (1.2) is non-maximal, and in $D=10$ type IIB supergravity. Furthermore, it sometimes occurs that the embedding (1.2) is maximal but non-symmetric, as in $D=11$ supergravity.

At any rate, we will show that the common features of the embedding (1.2) are at least two ( $c f r$. the start of appendix A):

- It is regular and preserves the rank of the group. Indeed, it generally holds that

$$
\operatorname{rank}\left(G_{N}^{3}\right)=\operatorname{rank}\left(G_{N}^{D}\right)+\operatorname{rank}(\operatorname{SL}(D-2, \mathbb{R}))=\operatorname{rank}\left(G_{N}^{D}\right)+D-3 .
$$

The same relation holds for the non-compact rank of these groups, namely the rank of the corresponding symmetric Riemannian manifolds of which the groups encode the isometries:

$$
\operatorname{rank}\left(\frac{G_{N}^{3}}{H_{N}^{3}}\right)=\operatorname{rank}\left(\frac{G_{N}^{D}}{H_{N}^{D}}\right)+\operatorname{rank}\left(\frac{\mathrm{SL}(D-2, \mathbb{R})}{\mathrm{SO}(D-2)}\right)=\operatorname{rank}\left(\frac{G_{N}^{D}}{H_{N}^{D}}\right)+D-3
$$

where $H_{N}^{3}$ and $H_{N}^{D}$ are the maximal compact subgroups of $G_{N}^{3}$ and $G_{N}^{D}$, respectively. As mentioned above, this does not imply the embeddings to be in general maximal nor symmetric.

- The pseudo-Riemannian coset resulting from (1.2) has always zero character [14, 15], namely it has the same number of compact and non-compact generators. We will show that this latter property is related to the Poincaré duality (alias electric-magnetic duality) of the spectrum of massless $p>0$ forms of the theory, which can essentially be traced back to the existence of an Hodge involution in the cohomology of the scalar manifold, singling out only the physical forms and their duals in the cohomology of the $(D-2)$-dimensional transverse space. This property also follows from the regularity of the embedding of $G_{N}^{D} \times \operatorname{SL}(D-2)$ inside $G_{N}^{3}$, the semisimplicity of the two groups and properties (1.3), (1.4), as it will be shown in appendix A.3.

There is also another aspect of interest in the present analysis: the role played by exceptional Lie groups and their relation to Jordan algebras and Freudenthal triple systems [19-21]. In particular, a mathematical construction, called Jordan pairs (see e.g. [22] for a recent treatment, and a list of refs.) corresponds to the maximal non-symmetric embedding

$$
E_{8(8)} \supset E_{6(6)} \times \mathrm{SL}(3, \mathbb{R}),
$$

\footnotetext{
${ }^{2}$ For enhancement to infinite symmetries, see [18].
} 
which is nothing but (1.2) specified for maximal supersymmetry $(N=16)$ and $D=5$. We point out that the Jordan pairs relevant for supergravity theories always pertain to suitable non-compact real forms of Lie algebras, differently e.g. from the treatment given in $[22]$.

Moreover, it is worth observing that in $D=11$ supergravity $G_{16}^{11}$ is empty, and thus (1.2) is the following maximal non-symmetric embedding [5]:

$$
E_{8(8)} \supset \mathrm{SL}(9, \mathbb{R})
$$

which in fact was used long time ago [23] in order to construct the gravity multiplet of this theory [24]. For maximal supergravity $\left(N=N_{\max }=16\right),(1.2)$ reads $^{3}$

$$
E_{8(8)} \supset E_{11-D(11-D)} \times \mathrm{SL}(D-2, \mathbb{R})
$$

where $G_{16}^{D}=E_{11-D(11-D)}$ denotes the so-called Cremmer-Julia sequence [8, 9]. The unique exception is provided by type IIB chiral $D=10$ supergravity, in which (1.2) is given by a two-step chain of maximal embeddings: ${ }^{4}$

$$
E_{8(8)} \supset_{s} \mathrm{SL}(2, \mathbb{R}) \times E_{7(7)} \supset_{s} \mathrm{SL}(2, \mathbb{R}) \times \mathrm{SL}(8, \mathbb{R}),
$$

which preserves the group rank.

The plan of the paper is as follows.

In section 2 we start by recalling some basic facts on $\mathrm{SO}(N)$ Clifford algebras relevant for the classification of massless multiplets of $\mathcal{N}$-extended supersymmetry in any dimension. Here $\mathcal{N}=2 N$ denotes the number of supersymmetries, regardless of the dimension $D$. Thus, for instance maximal supergravity corresponds to $\mathcal{N}=32$ (8 spinor supercharges in $D=4)$, whereas the minimal supergravity we consider has $\mathcal{N}=8$ (2 spinor supercharges in $D=4)$. We then proceed to considering the embedding (1.7) pertaining to maximal supergravity in any dimension $D \geqslant 4$ (in $D=10$ both IIA and IIB theories are considered). The embedding (1.2), which can be regarded as the "non-compact enhancement" of Nahm's analysis [23], in all cases consistently provides the massless spectrum of the corresponding theory with the correct spin-statistics content; illustrative analysis is worked out for $D=11$ and $D=10$ maximal theories. Other theories which do not exhibit matter coupling are also considered, namely $N=10,12$ in $D=4$ and $N=12$ in $D=5$.

In section 3 we consider half-maximal supergravity theories $(N=8)$, which can be matter coupled and exist in all $D \leqslant 10$ dimensions; for $D=6$ we consider both inequivalent theories, namely the chiral $(2,0)$ (type IIB) and the non-chiral $(1,1)$ (type IIA) ones. Theories with $N=6$, living in $D=4$, are also considered.

Then, in section 4 we consider quarter-maximal theories $(N=4)$, which live in $D=$ 4, 5, 6 and admit two different kinds of matter multiplets. We confine ourselves to theories with symmetric scalar manifolds, which (apart from the minimally coupled models in $D=4$

\footnotetext{
${ }^{3}$ This embedding was considered, but not proved, in [16]. A proof is presented in appendix A of the present paper.

4 "s" and " $n s "$ stand for "symmetric" and "non-symmetric" (embedding), respectively.
} 
and the non-Jordan symmetric sequence in $D=5$ ) admit an interpretation in terms of Euclidean Jordan algebras.

Pseudo-Riemannian cosets associated to the maximal-rank embeddings (1.2) are then analyzed in section 5. All such cosets enjoy the property of having the same number of compact and non-compact generators. This is also proven, using general group theoretical arguments, in appendix A.3. In subsection 5.2 this property is related to the invariance of the spectrum of massless bosonic $p>0$ forms under Poincaré-duality, or equivalently in subsection 5.3 in terms of an Hodge involution acting on the coset cohomology.

Final remarks and outlook are given in section 6 .

Three appendices conclude the paper. In appendix A, some embeddings of noncompact, real forms relevant for our analysis are rigorously proved, while in appendix B the issue of inequivalent "dual pairs" of subalgebras of the $U$-duality algebra is discussed (see also [25]). The related notions of $T$-dualities as $\mathfrak{s o}(8,8)$ outer-automorphisms are also dealt with. In appendix $\mathrm{C}$ the issue of Poincare duality is revisited with an explicit algebraic construction which makes use of appropriate level decompositions.

\section{Clifford algebras and "pure" theories}

In the seminal paper by Nahm [23], it was shown how massless multiplets of supergravity are built in terms of irreps. of $\mathrm{SO}(D-2)$, the little group (spin) of massless particles in $D$ dimensions. The number of supersymmetries $2 N$ is encoded in the Clifford algebra of $\mathrm{SO}(N)$, and therefore the supermultiplets can be regarded as $S O(N)$ spinors decomposed into $S O(D-2)$ irreps (for theories with particles with spin $s \leqslant 2$, which we consider throughout, $N_{\max }=16$ ). Bosons and fermions thus correspond to the two semi-spinors (or chiral spinors) of ${ }^{5} \mathrm{SO}(N)$.

In any dimension $D \geqslant 4, S O(N)$ exhibits a certain commuting factor with the massless little group $S O(D-2)$. For "pure" supergravities, in which only the gravity multiplet is present, such a commuting factor is the so-called $\mathcal{R}$-symmetry of the theory. Then the question arises as to which is the non-compact group commuting with the $\operatorname{SL}(D-2, \mathbb{R})$ Ehlers group (which thus extends the massless little group including the $\mathcal{R}$-symmetry), and furthermore which is the non-compact group which extends the $\mathrm{SO}(N)$ of the $N$ dimensional Clifford algebra pertaining to $2 N$ local supersymmetries.

In describing massless multiplets of theories with $\mathcal{N}=2 N$ local supersymmetries, one consider the the rest-frame supersymmetry algebra without central extension. Since the momentum squares to zero $\left(P^{\mu} P_{\mu}=0\right)$, only half of the supersymmetry charges survive, and the creation operators of $N$ charges describe an $\mathrm{SO}(N)$ Clifford algebra. Moreover, due to the fact that in $D \geqslant 4$ spinors always have real dimension multiple of $4, N$ is always even: $N=4,6,8,10,12,16$ (we do not consider here $N=2$ at $D=4$, namely minimal 4-dimensional supergravity with 1 spinor supercharge). It thus comes as no surprise that

\footnotetext{
${ }^{5}$ Note that $N$ is always even, since for $D \geqslant 4$ spinor charges have real dimensions multiples of 4 .

Furthermore, it should be remarked that the cases $D=4, N=2$ and $D=10, N=8$ are somewhat particular, because $N=D-2$, so the two Clifford spinors directly provide bosonic and fermionic supermultiplets' representations.
} 
$U$-duality groups $G_{N}^{3}$ in $D=3$ (in which there is only distinction between bosons and fermions, but no spin is present for massless states) contain in their mcs the Clifford algebra symmetry $\mathrm{SO}(N)$.

Supersymmetry dictates that massless bosons and fermions are simply the two (chiral, semi-) spinor irreps. of $\mathrm{SO}(N)$, while their spin $s$ content in $D$ space-time dimensions is obtained by suitably branching such irreps. into $\mathrm{SO}(D-2)$, which is the little group (spin) for massless particles in $D$ dimensions.

In the present section we consider "pure" theories in which the matter coupling is not allowed; they include maximally supersymmetric $(N=16)$ theories in any dimension $D \leqslant 11$, as well as non-maximal theories with $N=10,12$ in $D=4$ and $N=12$ in $D=5$. For such theories, the Clifford algebra $\mathrm{SO}(N)$ is nothing but the $m c s$ of the $U$-duality group $G_{N}^{3}$ in $D=3$; for non-maximal theories $(N<16)$, this is true up to the presence of the so-called Clifford vacuum factor group, which expresses further degeneracy of the Clifford algebra symmetry. Moreover, the group $H_{N}^{D}=m c s\left(G_{N}^{D}\right)$ which commutes with $\operatorname{SO}(D-2)$ inside $\mathrm{SO}(N)$ is the $\mathcal{R}$-symmetry, providing the degeneracy of the spin $s$ representations in the decomposition of the chiral spinors under the embedding ${ }^{6}[23]$

$$
S O(N) \supset H_{N}^{D} \times S O(D-2)_{J}
$$

which is the (not necessarily maximal-rank, nor maximal nor symmetric) counterpart of (1.2) at the level of $m c s$. The subscript " $J$ " denotes the spin group throughout.

\section{$2.1 \quad N=16($ maximal $)$}

For maximal $(N=16)$ supergravity theories with massless particles, the $D=3 U$ duality group is $G_{3}^{16}=E_{8(8)}$, with $m c s \mathrm{SO}(16)$, which is the Clifford algebra for massless particles with $\mathcal{N}=32$ supersymmetries. (1.7) provides the rank-preserving embedding of $D$-dimensional Ehlers group $S L(D-2, \mathbb{R})$ into $E_{8(8)}$. The group commuting with $S L(D-2, \mathbb{R})$ inside $E_{8(8)}$ is nothing but the $D$-dimensional $U$-duality group $G_{16}^{D}=$ $E_{11-D(11-D)}$, belonging to the so-called the Cremmer-Julia sequence. All cases in $4 \leqslant D \leqslant$ 11 dimensions are reported in table 1 (non-compact level (1.2)-(1.7)) and in table 2 (mcs level (2.1)). In particular, in table 2 also the decomposition of the vector irrep. 16 of the Clifford algebra $\mathrm{SO}(16)=m c s\left(E_{8(8)}\right)$ of maximal $(N=16 \rightarrow \mathcal{N}=32)$ supersymmetry is reported for the embedding (2.1) pertaining to this case, namely [23] (see also [26]):

$$
\mathrm{SO}(16) \supset \mathcal{R}_{D}^{16} \times \mathrm{SO}(D-2)_{J}
$$

where, as mentioned above, $\mathcal{R}_{D}^{16} \equiv \operatorname{mcs}\left(G_{D}^{16}\right) \equiv H_{D}^{16}$ is the $\mathcal{R}$-symmetry of the maximal supergravity in $D$ (Lorentzian) space-time dimensions. Note that the irrep. of $\mathrm{SO}(D-2)$ occurring in the branching of the $\mathbf{1 6}$ along (2.2) are all spinors, and the $\mathcal{R}$-symmetry $\mathcal{R}_{D}^{16}$ is real, pseudo-real (quaternionic), complex, depending on whether such spinor irrep. is real, pseudo-real or complex, respectively.

\footnotetext{
${ }^{6}$ Further commuting factor group occurs in the l.h.s. of $(2.1)$ in non-maximal $(N \leqslant 16)$ theories; see analysis below.
} 


\begin{tabular}{|c|c|c|}
\hline$D$ & $E_{8(8)} \supset E_{11-D(11-D)} \times \mathrm{SL}(D-2, \mathbb{R})$ & type \\
\hline 11 & $E_{8(8)} \supset \mathrm{SL}(9, \mathbb{R})$ & $\max , n s$ \\
\hline $10, I I A$ & $E_{8(8)} \supset \mathrm{SO}(1,1) \times \mathrm{SL}(8, \mathbb{R})$ & $n m, n s$ \\
\hline $10, I I B$ & $E_{8(8)} \supset \mathrm{SL}(2, \mathbb{R}) \times \mathrm{SL}(8, \mathbb{R})$ & $n m, n s$ \\
\hline 9 & $E_{8(8)} \supset G L(2, \mathbb{R}) \times \operatorname{SL}(7, \mathbb{R})$ & $n m, n s$ \\
\hline 8 & $E_{8(8)} \supset \mathrm{SL}(2, \mathbb{R}) \times \mathrm{SL}(3, \mathbb{R}) \times \mathrm{SL}(6, \mathbb{R})$ & $n m, n s$ \\
\hline 7 & $E_{8(8)} \supset \mathrm{SL}(5, \mathbb{R}) \times \mathrm{SL}(5, \mathbb{R})$ & $\max , n s$ \\
\hline 6 & $E_{8(8)} \supset \mathrm{SO}(5,5) \times \mathrm{SL}(4, \mathbb{R})$ & $n m, n s$ \\
\hline 5 & $E_{8(8)} \supset E_{6(6)} \times \mathrm{SL}(3, \mathbb{R})$ & $\max , n s$ \\
\hline 4 & $E_{8(8)} \supset E_{7(7)} \times \mathrm{SL}(2, \mathbb{R})$ & $\max , s$ \\
\hline
\end{tabular}

Table 1. Embedding $G_{N}^{3} \supset G_{N}^{D} \times \operatorname{SL}(D-2, \mathbb{R})$ ( 1.2) for maximal supergravity theories $(N=16)$ in $11 \geqslant D \geqslant 4$ Lorentzian space-time dimensions $[16,17] . G_{N}^{D}$ is the $U$-duality group in $D$ dimensions for the theory with $\mathcal{N}=2 N$ supersymmetries. $\operatorname{SL}(D-2, \mathbb{R})$ is the Ehlers group in $D$ dimensions. For $N=16, G_{16}^{3}=E_{8(8)}$, and $G_{N}^{D}=E_{11-D(11-D)}$ belongs to the Cremmer-Julia sequence; thus, (1.7) is obtained. The type (max (imal), $n$ (ext-to-) $m$ (aximal), $s$ (ymmetric), $n$ (on-) $s$ (ymmetric)) of embedding is indicated. Explicit proofs are given in appendix A.

Let us scan them briefly (as anticipated, for $D=11$ and $D=10$ the massless spectrum analysis is also worked out, as an example of the consistence of the embeddings with the massless spectrum of the corresponding theory). For convenience of the reader, we anticipate that the embeddings (1.2) and (2.1) are maximal in $D=11,7,5$ (non-symmetric) 


\begin{tabular}{|c|c|c|}
\hline$D$ & $S O(16) \supset H_{16}^{D} \times \mathrm{SO}(D-2)_{J}$ & type \\
\hline 11 & $\begin{array}{c}S O(16) \supset \mathrm{SO}(9) \\
\mathbf{1 6}=\mathbf{1 6}\end{array}$ & $\max , n s$ \\
\hline $10, I I A$ & $\begin{array}{c}S O(16) \supset \mathrm{SO}(8) \\
\mathbf{1 6}=\mathbf{8}_{s}+\mathbf{8}_{c}\end{array}$ & $n m, n s$ \\
\hline $10, I I B$ & $\begin{array}{c}S O(16) \supset \mathrm{SO}(2) \times \mathrm{SO}(8) \\
\mathbf{1 6}=\left(\mathbf{2}, \mathbf{8}_{s}\right)\end{array}$ & $n m, n s$ \\
\hline 9 & $\begin{array}{c}S O(16) \supset \mathrm{SO}(2) \times \mathrm{SO}(7) \\
\mathbf{1 6}=(\mathbf{2}, \mathbf{8})\end{array}$ & $n m, n s$ \\
\hline 8 & $\begin{array}{c}\mathrm{SO}(16) \supset \mathrm{U}(1) \times \mathrm{SU}(2) \times \mathrm{SU}(4) \\
\mathbf{1 6}=(\mathbf{2}, \mathbf{4})+(\overline{\mathbf{2}}, \overline{\mathbf{4}})\end{array}$ & $n m, n s$ \\
\hline 7 & $\begin{array}{c}\mathrm{SO}(16) \supset \mathrm{USp}(4) \times \mathrm{USp}(4) \\
\mathbf{1 6}=(\mathbf{4}, \mathbf{4})\end{array}$ & $\max , n s$ \\
\hline 6 & $\begin{array}{c}\mathrm{SO}(16) \supset \mathrm{USp}(4)_{L} \times \mathrm{USp}(4)_{R} \times \mathrm{SU}(2)_{L} \times \mathrm{SU}(2)_{R} \\
\mathbf{1 6}=(\mathbf{4}, \mathbf{1}, \mathbf{2}, \mathbf{1})+(\mathbf{1}, \mathbf{4}, \mathbf{1}, \mathbf{2})\end{array}$ & $n m, n s$ \\
\hline 5 & $\begin{array}{c}\mathrm{SO}(16) \supset \mathrm{USp}(8) \times \mathrm{SU}(2) \\
\mathbf{1 6}=(\mathbf{8}, \mathbf{2})\end{array}$ & $\max , n s$ \\
\hline 4 & $\begin{aligned} \mathrm{SO}(16) & \supset \mathrm{SU}(8) \times \mathrm{U}(1) \\
\mathbf{1 6} & =\mathbf{8}_{1}+\overline{\mathbf{8}}_{-1}\end{aligned}$ & $\max , s$ \\
\hline
\end{tabular}

Table 2. Embedding $H_{N}^{3} \supset H_{N}^{D} \times \mathrm{SO}(D-2)$ ( 2.1) [23] for maximal supergravity theories $(N=16)$ in $11 \geqslant D \geqslant 4$ Lorentzian space-time dimensions. In this case, as for all "pure" theories, $H_{N}^{D}$ is the $\mathcal{R}$-symmetry for the theory with $\mathcal{N}=2 N$ supersymmetries. $\mathrm{SO}(D-2)$ is the little group (spin group) for massless particles. In this case, $H_{16}^{3}=\mathrm{SO}(16)$ is the Clifford algebra of maximal supersymmetry.

and 4 (symmetric), while they are next-to-maximal in $D=10,9,8,6$; in these latter cases, an "enhancement" of $E_{11-D(11-D)} \times S L(D-2, \mathbb{R})$ occurs (see analysis below).

1. $D=11$ ( $M$-theory). There is no continuous $U$-duality (and thus $\mathcal{R}$-symmetry) group, and (1.7) specifies to (1.6), namely the maximal non-symmetric embedding of 
the Ehlers group $\mathrm{SL}(9, \mathbb{R})$ only:

$$
\begin{aligned}
& E_{8(8)} \supset_{n s} \mathrm{SL}(9, \mathbb{R}) ; \\
& \mathbf{2 4 8}=\mathbf{8 0}+\mathbf{8 4}+\mathbf{8 4}^{\prime},
\end{aligned}
$$

where $\mathbf{8 4}$ and $\mathbf{8 4}^{\prime}$ are the 3 -fold antisymmetric of $\mathrm{SL}(9, \mathbb{R})$ and its dual; they correspond to gauge fields coupling to $M 2$ branes and $M 5$ branes, respectively. The corresponding mcs level is given by the specification of (2.1) to the following nonsymmetric embedding of the massless spin group $S O(9)$ only:

$$
S O(16) \supset_{n s} S O(9) \text {. }
$$

For what concerns the massless spectrum, one considers the maximal symmetric embedding $^{7}$

$$
E_{8(8)} \stackrel{m c s}{\supset} S O(16): \mathbf{2 4 8}=\mathbf{1 2 0}+\mathbf{1 2 8},
$$

where 128 is one of the two chiral spinor irreps. of $S O(16)$. Under (2.4), such two chiral irreps. $\mathbf{1 2 8}$ and $\mathbf{1 2 8}^{\prime}$ further decompose as follows:

$$
S O(16) \supset_{n s} S O(9):\left\{\begin{array}{l}
\mathbf{1 2 8}=\mathbf{8 4}+\mathbf{4 4} \\
\mathbf{1 2 8}^{\prime}=\mathbf{1 2 8}
\end{array}\right.
$$

where, on the right-hand side, $\mathbf{4 4 , 8 4}$ and $\mathbf{1 2 8}$ are the rank-2 symmetric traceless, the rank-3 antisymmetric and the gamma-traceless vector-spinor irreps. of the massless spin group $\mathrm{SO}(9)$, respectively. Thus, (2.6) establishes the chiral spinor irrep. 128 of the Clifford algebra $\mathrm{SO}(16)$ to be irrep. pertaining to the massless bosonic spectrum (it branches into the graviton 44 and the 3-form 84), whereas its conjugate semi-spinor irrep. $\mathbf{1 2 8}^{\prime}$ pertains to the massless fermionic spectrum of $M$-theory (it corresponds to the $D=11$ gravitino).

2. In $D=10$ type IIA theory the $U$-duality is $G_{16}^{10 I I A}=\mathrm{SO}(1,1)$ (and thus no continuous $\mathcal{R}$-symmetry); since this theory is obtained as the Kaluza-Klein $S^{1}$ reduction of $M$-theory, the relevant chain of maximal embeddings reads

$$
E_{8(8)} \supset_{n s} \mathrm{SL}(9, \mathbb{R}) \supset_{s} \mathrm{SO}(1,1) \times \mathrm{SL}(8, \mathbb{R}) ;
$$

note the "enhancement" to $\operatorname{SL}(9, \mathbb{R})$, consistent with the $M$-theoretical origin of IIA theory. The corresponding mcs level is

$$
S O(16) \supset_{n s} \mathrm{SO}(9) \supset_{s} \mathrm{SO}(8),
$$

where $\mathrm{SO}(8)$ is the massless spin group. Throughout our analysis, we dub "nextto-maximal" $(\mathrm{nm})$ those embeddings given by a chain of two maximal embeddings; note that all $\mathrm{nm}$ embeddings considered in the present investigation are of maximal rank, namely they preserve the rank of the original group. For what concerns the IIA

\footnotetext{
${ }^{7}$ For further subtleties concerning exceptional Lie algebras, see [25] and appendix B further below.
} 
massless spectrum, one considers the branchings of $\mathbf{1 2 8}$ (bosons) and $\mathbf{1 2 8}$ (fermions) of the Clifford algebra $S O(16)$ under the $n m$ embedding (2.8):

$$
\begin{aligned}
\mathbf{1 2 8} & =\mathbf{8 4}+\mathbf{4 4}=\mathbf{5 6} \mathbf{6}_{v}+\mathbf{2 8}+\mathbf{3 5} 5_{v}+\mathbf{8}_{v}+\mathbf{1} ; \\
128^{\prime} & =\mathbf{1 2 8}=\mathbf{5} \boldsymbol{6}_{s}+\mathbf{5 6} \boldsymbol{6}_{c}+\mathbf{8}_{s}+\mathbf{8}_{c},
\end{aligned}
$$

where the subscripts " $v$ ", "s" and " $c$ " respectively stand for vector, spinor, conjugate spinor, and they pertain to the triality of $\mathrm{SO}(8)$, the little group (spin group) of massless particles in $D=10 . \mathbf{5 6}_{i}, \mathbf{2 8}, \mathbf{3 5}_{i}$ and $\mathbf{8}_{i}(i=v, s, c)$ are the rank-3 antisymmetric, adjoint, rank-2 symmetric traceless and vector/spinor irreps. of $\mathrm{SO}(8)$, respectively. Thus, the branching (2.9) consistently pertains to the IIA massless bosonic spectrum: 3-form $C_{\mu \nu \rho}^{(3)}\left(\mathbf{5 6}_{v}\right), B$-field $B_{\mu \nu}(\mathbf{2 8})$, graviton $g_{\mu \nu}\left(\mathbf{3 5}_{v}\right)$, graviphoton $C_{\mu}^{(1)}\left(\mathbf{8}_{v}\right)$ and dilaton scalar field $\phi_{10}(\mathbf{1})$. On the other hand, the branching $(2.10)$ pertains to the IIA massless fermionic spectrum: gravitinos $\mathbf{5 6}_{s}$ and $\mathbf{5 6}_{c}(s=3 / 2$ Majorana-Weyl spinors of opposite chirality), and gauginos $\boldsymbol{8}_{s}$ and $\boldsymbol{8}_{c}(s=1 / 2$ Majorana-Weyl spinors of opposite chirality). This non-chiral spectrum can also be deduced by dimensional reduction of the maximal supersymmetric supermultiplet of $D=11$ supergravity (M-theory).

3. On the other hand, in $D=10$ type IIB theory the $U$-duality is $G_{16}^{10}{ }^{I I B}=\operatorname{SL}(2, \mathbb{R})$, and its $m c s$ is the $\mathcal{R}$-symmetry $\mathrm{U}(1)$, and the relevant $n m$ embedding is given by (1.8), which we report here:

$$
\begin{aligned}
E_{8(8)} & \supset_{s} \mathrm{SL}(2, \mathbb{R}) \times E_{7(7)} \supset_{s} \mathrm{SL}(2, \mathbb{R}) \times \mathrm{SL}(8, \mathbb{R}) ; \\
S O(16) & \supset_{s} \mathrm{U}(1) \times \mathrm{SU}(8) \supset_{s} \mathrm{U}(1) \times \mathrm{SO}(8) ;
\end{aligned}
$$

note the "exceptional enhancement" to $E_{7(7)}$ in (2.11). For what concerns the IIB massless spectrum, one considers the branching of 128 (bosons) and $\mathbf{1 2 8}^{\prime}$ (fermions) of the Clifford algebra $S O(16)$ under the $n m$ embedding (2.12). Under the decomposition

$$
\begin{aligned}
\mathrm{SU}(8) & \supset_{s} \mathrm{SO}(8) \\
\mathbf{8} & =\mathbf{8}_{s},
\end{aligned}
$$

one obtains (disregarding $\mathrm{U}(1)$ charges)

$$
\begin{aligned}
& 128=70_{0}+\mathbf{2 8}+\overline{\mathbf{2 8}}+\mathbf{1}+\mathbf{1}=35_{v}+35_{c}+\mathbf{2 8}+\mathbf{2 8}+\mathbf{1}+\mathbf{1} ; \\
& 128^{\prime}=\mathbf{5 6}+\overline{\mathbf{5 6}}+\mathbf{8}+\overline{\mathbf{8}}=\mathbf{5 6} 6_{s}+\mathbf{5 6} 6_{s}+\mathbf{8}_{s}+\mathbf{8}_{s} .
\end{aligned}
$$

Note that, upon (2.13), the rank-4 antisymmetric self-real irrep. 70 of SU(8) breaks into $\mathbf{3 5} \mathbf{5}_{v}+\mathbf{3 5}_{c}$ of $\mathrm{SO}(8)$. Thus, the branching (2.14) consistently pertains to the IIB massless bosonic spectrum: graviton $g_{\mu \nu}\left(\mathbf{3 5}_{v}\right)$, 4-form $C^{(4)}\left(\mathbf{3 5}_{c}\right), B$-field $B_{\mu \nu}$ (28), 2-form $C_{\mu \nu}^{(2)}(\mathbf{2 8})$, and two scalar fields, namely the dilaton $\phi_{10}$ and the axion $C^{(0)}(\mathbf{1}+\mathbf{1})$. On the other hand, the branching $(2.15)$ pertains to the IIB massless fermionic spectrum: gravitinos $\mathbf{5 6}_{s}$ and $\mathbf{5 6}_{s}(s=3 / 2$ Majorana-Weyl spinors of same chirality) and gauginos $\boldsymbol{8}_{s}$ and $\boldsymbol{8}_{s}$ ( $s=1 / 2$ Majorana-Weyl spinors of same chirality). This spectrum is chiral and hence cannot be obtained by dimensional reduction of the $D=11 M$-theory supermultiplet. 
4. In $D=9$ the $U$-duality is $G_{16}^{9}=G L(2, \mathbb{R}) \equiv E_{2(2)}$, and its $m c s$ is the $\mathcal{R}$-symmetry $\mathrm{U}(1)$. There are two possible chains of maximal embeddings, which are equivalent up to redefinitions of $\mathrm{SO}(1,1)$ weights. The first chain, pertinent to a dimensional reduction of $M$-theory, gives rise to a $n m$ embedding:

$$
\begin{aligned}
E_{8(8)} & \supset_{n s} \mathrm{SL}(9, \mathbb{R}) \supset_{s} G L(2, \mathbb{R}) \times \mathrm{SL}(7, \mathbb{R}) ; \\
\mathrm{SO}(16) & \supset_{n s} \mathrm{SO}(9) \supset_{s} \mathrm{U}(1) \times \mathrm{SO}(7),
\end{aligned}
$$

whereas the second, pertaining to a Kaluza-Klein $S^{1}$-reduction of $D=10$ IIB theory, determines a "next-to-next-to-maximal" ( $n n m$ ) embedding, because it is 3 -stepwise (it is given by a further branching of IIB chain (2.11)):

$$
\begin{aligned}
E_{8(8)} & \supset_{s} \mathrm{SL}(2, \mathbb{R}) \times E_{7(7)} \supset_{s} \mathrm{SL}(2, \mathbb{R}) \times \mathrm{SL}(8, \mathbb{R}) \supset_{s} G L(2, \mathbb{R}) \times \mathrm{SL}(7, \mathbb{R}) ; \\
S O(16) & \supset_{s} \mathrm{U}(1) \times \mathrm{SU}(8) \supset_{s} \mathrm{U}(1) \times \mathrm{SO}(8) \supset_{s} \mathrm{U}(1) \times \mathrm{SO}(7)
\end{aligned}
$$

Besides being equivalent, (2.16)-(2.17) and (2.18)-(2.19) are consistent, because type IIA and IIB theories are equivalent in $D \leqslant 9$ dimensions (except for half-maximal supergravity in $D=6$; see further below).

5. In $D=8$ the $U$-duality is $G_{16}^{8}=\mathrm{SL}(2, \mathbb{R}) \times \mathrm{SL}(3, \mathbb{R}) \equiv E_{3(3)}$, and its $m c s$ is the $\mathcal{R}$ symmetry $\mathrm{U}(1) \times \mathrm{SU}(2) \sim \mathrm{U}(2)$. The relevant $n m$ embedding reads ${ }^{8}(\mathrm{SO}(6) \sim \mathrm{SU}(4))$

$$
\begin{aligned}
& E_{8(8)} \supset_{n s} E_{6(6)} \times \mathrm{SL}(3, \mathbb{R}) \supset_{s} \mathrm{SL}(2, \mathbb{R}) \times \mathrm{SL}(3, \mathbb{R}) \times \mathrm{SL}(6, \mathbb{R}) ; \\
& \mathrm{SO}(16) \supset_{n s} \mathrm{USp}(8) \times \mathrm{SU}(2) \supset_{s} \mathrm{U}(1) \times \mathrm{SU}(2) \times \mathrm{SU}(4) \text {; }
\end{aligned}
$$

note the "exceptional enhancement" to $E_{6(6)}$ in (2.20).

6. In $D=7$ the $U$-duality is $G_{16}^{7}=\mathrm{SL}(5, \mathbb{R}) \equiv E_{4(4)}$, and its $m c s$ is the $\mathcal{R}$-symmetry $\mathrm{SO}(5) \sim \mathrm{USp}(4)$. The relevant embedding is maximal non-symmetric:

$$
\begin{aligned}
E_{8(8)} & \supset_{n s} S L(5, \mathbb{R}) \times \mathrm{SL}(5, \mathbb{R}) ; \\
\mathrm{SO}(16) & \supset_{n s} \mathrm{USp}(4) \times \mathrm{USp}(4) .
\end{aligned}
$$

Note that in this case there is perfect symmetry between the $\mathcal{R}$-symmetry and the massless spin sectors.

7. In $D=6$ (non-chiral $(2,2)$ ) maximal theory, the $U$-duality is $G_{16}^{6}=\mathrm{SO}(5,5) \equiv E_{5(5)}$, and its $m c s$ is the $\mathcal{R}$-symmetry ${ }^{9} \mathrm{SO}(5) \times \mathrm{SO}(5) \sim \mathrm{USp}(4)_{L} \times \mathrm{USp}(4)_{R}$. The relevant

${ }^{8}(2.21)$ is the $n=4$ case of the maximal non-symmetric embedding pattern

$$
\begin{aligned}
& S O(4 n) \supset_{n s} S U(2) \times U S p(2 n) ; \\
& \mathbf{A d j}_{\mathrm{SO}(4 n)}=\mathbf{A d \mathbf { j } _ { \mathrm { SU } ( 2 ) }}+\mathbf{A} \mathbf{d j}_{\mathrm{USp}(2 n)}+\left(\mathbf{3}, \mathbf{A}_{2,0}\right) \text {, }
\end{aligned}
$$

where $\mathbf{A}_{2,0}$ is the rank-2 antisymmetric skew-traceless irrep. of $U S p(2 n)$. For the first appearance of such an embedding in supersymmetry, see [27].

${ }^{9}$ Subscripts " $L$ " and " $R$ " denote left and right chirality, respectively. 
$n m$ embedding reads $(\mathrm{SO}(4) \sim \mathrm{SU}(2) \times \mathrm{SU}(2))$

$$
\begin{aligned}
E_{8(8)} & \supset_{s} S O(8,8) \supset_{s} S O(5,5) \times S O(3,3) \sim S O(5,5) \times S L(4, \mathbb{R}) ; \\
\mathrm{SO}(16) & \supset_{s} \mathrm{SO}(8) \times \mathrm{SO}(8) \supset_{s} S O(5)_{L} \times S O(3) \times S O(5)_{R} \times S O(3) \\
& \sim \mathrm{USp}(4)_{L} \times \mathrm{USp}(4)_{R} \times \mathrm{SU}(2)_{L} \times \mathrm{SU}(2)_{R} ;
\end{aligned}
$$

note the "enhancement" to $\mathrm{SO}(8,8)$ in $(2.24)$. Note that in this case both the $\mathcal{R}$ symmetry and massless spin groups factorize in the direct product of opposite chiralities identical factors. The corresponding Jordan algebra interpretation of (2.24) is as follows:

$$
Q \operatorname{Conf}\left(J_{3}^{\mathbb{O}_{s}}\right) \supset \operatorname{Str}_{0}\left(J_{2}^{\mathbb{O}_{s}}\right) \times S L(4, \mathbb{R}),
$$

where $J_{3}^{\mathbb{Q}_{s}}$ and $J_{2}^{\mathbb{Q}_{s}} \sim \boldsymbol{\Gamma}_{5,5}$ are the rank-2 and rank-3 Euclidean Jordan algebras over the split octonions $\mathbb{O}_{s}$, and $Q C o n f$ and $S t r_{0}$ respectively denote the quasi-conformal and reduced structure groups ${ }^{10}$ (see e.g. [21] and refs. therein).

8. In $D=5$ the $U$-duality undergoes an exceptional enhancement: $G_{16}^{5}=E_{6(6)}$, and its $m c s$ is the $\mathcal{R}$-symmetry $\operatorname{USp}(8)$. The relevant embedding is maximal non-symmetric, and it is given by (1.5), which we report here (note that it is the first step of $n m$ embedding (2.20)-(2.21)):

$$
\begin{aligned}
E_{8(8)} & \supset_{n s} E_{6(6)} \times \mathrm{SL}(3, \mathbb{R}) ; \\
\mathrm{SO}(16) & \supset_{n s} \mathrm{USp}(8) \times \mathrm{SU}(2) .
\end{aligned}
$$

The corresponding Jordan algebra interpretation of (2.27) is as follows:

$$
Q \operatorname{Conf}\left(J_{3}^{\mathbb{O}_{s}}\right) \supset \operatorname{Str}_{0}\left(J_{3}^{\mathbb{Q}_{s}}\right) \times S L(3, \mathbb{R}),
$$

and it is a particular non-compact, real version of the Jordan-pair embeddings of exceptional Lie algebras recently considered in [22]. Note that the $\mathrm{SU}(2)$ in (2.28) is the principal $\mathrm{SU}(2)$ in $\mathrm{SL}(3, \mathbb{R})$ in $(2.27)$.

9. In $D=4$ the $U$-duality is $G_{16}^{4}=E_{7(7)}$, and its $m c s$ is the $\mathcal{R}$-symmetry $\mathrm{SU}(8)$. The relevant embedding is maximal symmetric (note that it is the first step of chains (2.11)(2.12) and (2.18)-(2.19)):

$$
\begin{aligned}
E_{8(8)} & \supset_{s} E_{7(7)} \times \mathrm{SL}(2, \mathbb{R}) ; \\
\mathrm{SO}(16) & \supset_{s} \mathrm{SU}(8) \times \mathrm{U}(1) .
\end{aligned}
$$

The corresponding Jordan algebra interpretation of (2.30) is as follows:

$$
Q \operatorname{Conf}\left(J_{3}^{\mathbb{Q}_{s}}\right) \supset \operatorname{Conf}\left(J_{3}^{\mathbb{O}_{s}}\right) \times S L(2, \mathbb{R}),
$$

where Conf denotes the conformal group of $J_{3}^{\mathbb{Q}_{s}}$ (see e.g. [21] and refs. therein). Similar Jordan-algebraic interpretations can be given for other supergravities in various dimensions.

\footnotetext{
${ }^{10}$ In theories related to Euclidean Jordan algebras $J_{3}$ of rank 3, the quasi-conformal $Q C o n f\left(J_{3}\right)$, conformal Conf $\left(J_{3}\right)$ and reduced structure $\operatorname{Str}_{0}\left(J_{3}\right)$ groups are the $U$-duality groups in $D=3,4$ and 5 dimensions, respectively. In particular, $\operatorname{Conf}\left(J_{3}\right)$ is nothing but the automorphism group Aut $\left(\mathfrak{M}\left(J_{3}\right)\right)$ of the corresponding Freudenthal triple system [19-21].
} 


\section{$2.2 \quad N=12$}

In the "pure" theory with $N=12$, the $D=3 U$-duality group is $G_{12}^{3}=E_{7(-5)}$, with $m c s$ $\mathrm{SO}(12) \times \mathrm{SU}(2)_{C V}$, where $\mathrm{SO}(12)$ is the Clifford algebra for massless particles with $\mathcal{N}=24$ supersymmetries. The $\mathrm{SU}(2)_{C V}$ factor pertains to the so-called Clifford vacuum $(C V)$, which is generally present for non-maximal theories $(N<16)$, and it indicates further degeneracy of the Clifford algebra symmetry. In this case, $\mathrm{SU}(2)_{C V}$ can be also explained by recalling that this theory shares the very same bosonic sector of a matter-coupled supergravity with $N=4[19,20]$, in which it is the $\mathcal{R}$-symmetry of the hypermultiplets' sector.

This theory can consistently be uplifted only to $D=4$ and $D=5$.

1. In $D=5$ the $U$-duality is $G_{12}^{5}=S U^{*}(6)$, and its mcs is the $\mathcal{R}$-symmetry $\operatorname{USp}(6)$. The relevant embedding is maximal non-symmetric:

$$
\begin{aligned}
E_{7(-5)} & \supset_{n s} S U^{*}(6) \times S L(3, \mathbb{R}) ; \\
\mathrm{SO}(12) \times \mathrm{SU}(2)_{C V} & \supset_{n s} \mathrm{USp}(6) \times \mathrm{SU}(2)_{J},
\end{aligned}
$$

where we introduced the subscript " $J$ " in order to discriminate between the Clifford vacuum $\mathrm{SU}(2)_{C V}$ and the $\mathrm{SU}(2)_{J}$ pertaining to the massless spin group in $D=5$. Note that the embedding (2.33) is maximal non-symmetric, while the embedding (2.34) is non-maximal non-symmetric.

2. In $D=4$ the $U$-duality is $G_{12}^{4}=S O^{*}(12)$, and its $m c s$ is the $\mathcal{R}$-symmetry $\mathrm{U}(6)$. The relevant embedding is maximal symmetric:

$$
\begin{array}{r}
E_{7(-5)} \supset_{s} S O^{*}(12) \times S L(2, \mathbb{R}) ; \\
\mathrm{SO}(12) \times \mathrm{SU}(2)_{C V} \supset_{s} \mathrm{SU}(6) \times \mathrm{U}(1) \times \mathrm{U}(1)_{J},
\end{array}
$$

and it pertains to the so-called $c^{*}-$ map (see e.g. [29], and refs. therein).

\section{$2.3 \quad N=10$}

In the "pure" theory with $N=10$, the $D=3 U$-duality group is $G_{10}^{3}=E_{6(-14)}$, with $m c s$ $\mathrm{SO}(10) \times \mathrm{SO}(2)_{C V}$, where $\mathrm{SO}(10)$ is the Clifford algebra for massless particles with $\mathcal{N}=20$ supersymmetries. In this case, $\mathrm{SO}(2)_{C V}$ can be also explained as [add...]

This theory can be uplifted only to $D=4$, in which the $U$-duality is $G_{10}^{4}=\mathrm{SU}(5,1)$, and its $m c s$ is the $\mathcal{R}$-symmetry $\mathrm{U}(5)$. The relevant embedding is maximal symmetric:

$$
\begin{array}{r}
E_{6(-14)} \supset_{s} \mathrm{SU}(5,1) \times S L(2, \mathbb{R}) ; \\
\mathrm{SO}(10) \times \mathrm{SO}(2)_{C V} \supset_{s} \mathrm{SU}(5) \times \mathrm{U}(1) \times \mathrm{U}(1)_{J} .
\end{array}
$$

\section{$3 \quad N=8,6$ matter coupled theories}

\section{$3.1 \quad N=8$}

Half-maximal theories with $N=8$ exist in $3 \leqslant D \leqslant 10$; moreover, for $D=6$ two inequivalent theories exist, i.e. the non-chiral IIA $(1,1)$ and the chiral IIB $(2,0)$. 
The $D=3 U$-duality group is $G_{8}^{3}=S O(8, D-2+m)$, where $m$ is the number of matter multiplets in $D=3$ other than those coming from the reduction of the gravity multiplet in $D$ dimensions. Furthermore, $\operatorname{mcs}\left(G_{8}^{3}\right)=\mathrm{SO}(8) \times S O(D-2+m)_{C V}$, where $\mathrm{SO}(8)$ is the Clifford algebra for massless particles with $\mathcal{N}=16$ supersymmetries, and $S O(D-2+m)_{C V}$ is the Clifford vacuum symmetry.

The relevant chain of maximal embeddings leading to the embedding of the $D$ dimensional Ehlers group $S L(D-2, \mathbb{R})$ into $S O(8, D-2+m)$ depends on the dimension and on the type of theory. We anticipate that embeddings (1.2) and (2.1) are maximal in $D=4$ (symmetric) and next-to-maximal in $5 \leqslant D \leqslant 10$.

- For $D \geqslant 5$ (and $D=6$ type IIA $(1,1)$ ), it is given by the following chain of two maximal symmetric steps:

$$
\begin{aligned}
& S O(8, D-2+m) \supset_{s} S O(D-2, D-2) \times S O(10-D, m) \\
& \supset_{s} S L(D-2, \mathbb{R}) \times \mathrm{SO}(1,1) \times S O(10-D, m)
\end{aligned}
$$

The group commuting with $S L(D-2, \mathbb{R})$ inside $S O(8, D-2+m)$ is nothing but the $D$-dimensional $U$-duality group $G_{8}^{D}=\mathrm{SO}(1,1) \times S O(10-D, m)$. Note the "enhancement" to $S O(D-2, D-2) \times S O(10-D, m)$. Furthermore, it is worth remarking that also for $m=0$ the Clifford vacuum degeneracy is still present with an $S O(D-2)_{C V}$ factor; this is an extra spin quantum number carried by the $\mathrm{SO}(8)$ Clifford algebra spinor. In fact, by considering the mcs level of the chain (3.1), one obtains

$$
\begin{aligned}
& S O(8)_{\text {Clifford }} \times S O(D-2+m)_{C V} \\
& \supset_{s} S O(D-2) \times S O(D-2)_{C V} \times S O(10-D) \times S O(m)_{C V} \\
& \supset_{s} S O(D-2)_{J} \times S O(10-D)_{\mathcal{R}} \times S O(m)_{C V},
\end{aligned}
$$

where the $D$-dimensional massless spin group $S O(D-2)_{J}=\operatorname{mcs}(S L(D-2, \mathbb{R}))$ is diagonally embedded into $S O(D-2) \times S O(D-2)_{C V}$, and the $\mathcal{R}$-symmetry is $\mathrm{SO}(10-D)$. $S O(m)_{C V}$ is the part of Clifford vacuum symmetry due to matter coupling.

- For $D=4$, the maximal symmetric embedding reads:

$$
S O(8,2+m) \supset_{s} S O(2,2) \times S O(6, m) \sim \mathrm{SL}(2, \mathbb{R})_{\text {Ehlers }} \times \mathrm{SL}(2, \mathbb{R}) \times S O(6, m),
$$

and it pertains to the so-called $c^{*}$-map (see e.g. [29], and refs. therein). The group commuting with $\operatorname{SL}(2, \mathbb{R})_{\text {Ehlers }}$ inside $S O(8,2+m)$ is the 4-dimensional $U$-duality group $G_{8}^{4}=\operatorname{SL}(2, \mathbb{R}) \times S O(6, m)$. Also in this case for $m=0$ the Clifford vacuum degeneracy is still present with an $S O(2)_{C V}$ factor. In fact, by considering the mcs level of (3.3), one obtains the following maximal symmetric embedding $(\mathrm{SO}(6) \sim$ $\mathrm{SU}(4))$ :

$$
\begin{aligned}
& S O(8)_{\text {Clifford }} \times S O(2+m)_{C V} \\
& \supset_{s} S O(2)_{J} \times S O(2)_{C V} \times S O(6) \times \mathrm{SO}(m)_{C V} \sim U(1)_{J} \times U(4)_{\mathcal{R}} \times \mathrm{SO}(m)_{C V},
\end{aligned}
$$


where $S O(2)_{J}=\operatorname{mcs}\left(\mathrm{SL}(2, \mathbb{R})_{\text {Ehlers }}\right)$, and the $\mathcal{R}$-symmetry is $S O(2)_{C V} \times S O(6) \sim$ $\mathrm{U}(4)_{\mathcal{R}}$. Moreover, $S O(m)_{C V}$ is the part of Clifford vacuum symmetry due to matter coupling.

- For $D=6$ type IIB $(2,0)$, it suffices to start with $S O(8,3+m)$, and the maximal symmetric embedding reads as follows:

$$
S O(8,3+m) \supset_{s} S O(3,3) \times S O(5, m) \sim \mathrm{SL}(4, \mathbb{R}) \times S O(5, m) .
$$

The group commuting with $S L(4, \mathbb{R})$ inside $S O(8,4+m)$ is the 6-dimensional type IIB $U$-duality group $G_{8}^{6, I I B}=S O(5, m)$. The corresponding $m c s$ level reads

$$
\begin{aligned}
& S O(8)_{\text {Clifford }} \times S O(3+m)_{C V} \\
& \supset_{s}(S O(3) \times S O(3))_{J} \times S O(5)_{\mathcal{R}} \times \mathrm{SO}(m)_{C V} \sim \mathrm{SO}(4)_{J} \times U S p(4)_{\mathcal{R}} \times \mathrm{SO}(m)_{C V},
\end{aligned}
$$

where $\mathrm{SO}(4)_{J}=m c s\left(\mathrm{SL}(4, \mathbb{R})_{\text {Ehlers }}\right)$, and the $\mathcal{R}$-symmetry is $\mathrm{SO}(5) \sim \mathrm{USp}(4)$. Furthermore, $S O(m)_{C V}$ is the part of Clifford vacuum symmetry due to matter coupling.

All cases in $4 \leqslant D \leqslant 10$ dimensions are reported in tables 3 and 4 .

\section{$3.2 N=6$}

Theories with $N=6$ exist only in $D=3,4$.

The $D=3 U$-duality group is $G_{6}^{3}=\mathrm{SU}(4, m+1)$, where $m$ is the number of matter multiplets in $D=3$ other than those coming from the reduction of the gravity multiplet in 4 dimensions. Furthermore, $m c s\left(G_{6}^{3}\right)=\mathrm{SU}(4) \times U(m+1)_{C V}$, where $\mathrm{SU}(4) \sim \mathrm{SO}(6)$ is the Clifford algebra for massless particles with $\mathcal{N}=12$ supersymmetries, and $U(m+1)_{C V}$ is the Clifford vacuum symmetry.

The embedding of the 4-dimensional Ehlers group $S L(2, \mathbb{R})$ into $\mathrm{SU}(4, m+1)$ is maximal and symmetric:

$$
\mathrm{SU}(4, m+1) \supset_{s} \mathrm{SL}(2, \mathbb{R}) \times \mathrm{U}(3, m),
$$

and at the mcs level:

$$
\mathrm{SU}(4) \times S U(m+1) \times \mathrm{U}(1) \supset_{s} \mathrm{U}(1)_{J} \times \mathrm{U}(3) \times \mathrm{U}(m),
$$

where $D=4 U$-duality group is $G_{6}^{4}=\mathrm{SU}(3, m)$, and the $\mathcal{R}$-symmetry is $\mathrm{U}(3) . \mathrm{U}(m)$ is the $D=4$ Clifford vacuum symmetry, which is related to the number of matter multiplets.

\section{$4 \quad N=4$ matter coupled symmetric theories}

Quarter-maximal theories with $N=4$ exist in $3 \leqslant D \leqslant 6$; in particular, in $D=6$ they are chiral $(1,0)$ theories. The new feature of $N=4$ theories is the possible existence of two different types of matter multiplets, namely vector and hyper multiplets, transforming in different ways under the $\mathcal{R}$-symmetry, which is $\mathrm{U}(2)$ in $D=4$ and $\operatorname{USp}(2)$ in $D=5,6$. 


\begin{tabular}{|c|c|c|}
\hline$D$ & $S O(8, D-2+m) \supset G_{D}^{8}(m) \times \mathrm{SL}(D-2, \mathbb{R})$ & type \\
\hline 10 & $S O(8,8+m) \supset \mathrm{SO}(1,1) \times S O(m) \times S L(8, \mathbb{R})$ & $n m, n s$ \\
\hline 9 & $S O(8,7+m) \supset \mathrm{SO}(1,1) \times S O(1, m) \times S L(7, \mathbb{R})$ & $n m, n s$ \\
\hline 8 & $S O(8,6+m) \supset \mathrm{SO}(1,1) \times S O(2, m) \times S L(6, \mathbb{R})$ & $n m, n s$ \\
\hline 7 & $S O(8,5+m) \supset \mathrm{SO}(1,1) \times S O(3, m) \times S L(5, \mathbb{R})$ & $n m, n s$ \\
\hline $6, I I A$ & $S O(8,4+m) \supset \mathrm{SO}(1,1) \times S O(4, m) \times \mathrm{SL}(4, \mathbb{R})$ & $n m, n s$ \\
\hline $6, I I B$ & $S O(8,3+m) \supset S O(5, m) \times \mathrm{SL}(4, \mathbb{R})$ & $\max , s$ \\
\hline 5 & $S O(8,3+m) \supset \mathrm{SO}(1,1) \times S O(5, m) \times \mathrm{SL}(3, \mathbb{R})$ & $n m, n s$ \\
\hline 4 & $S O(8,2+m) \supset(\mathrm{SL}(2, \mathbb{R}) \times S O(6, m)) \times \mathrm{SL}(2, \mathbb{R})$ & $\max , s$ \\
\hline
\end{tabular}

Table 3. Embedding $G_{8}^{3} \supset G_{8}^{D} \times \operatorname{SL}(D-2, \mathbb{R})$ ( 1.2) for half-maximal supergravity theories $(N=8)$ in $10 \geqslant D \geqslant 4$ Lorentzian space-time dimensions.

In the following treatment, we will only consider theories based on symmetric Abelianvector multiplets' scalar manifolds, which is a restriction to $D=4$ (Kähler) and $D=5$ (real) special geometry; these theories will be denoted as ${ }^{11}$ symmetric $N=4$ theories.

In $D=4,5$, symmetric theories are classified by two infinite sequences, as well as by isolated cases given by the so-called "magical" models.

We will also not consider the ( $D$-independent) hypermultiplets' quaternionic scalar manifolds.

\footnotetext{
${ }^{11}$ We will not consider here the so-called non-Jordan symmetric sequence (see e.g. [30] and refs. therein) in $D=5$, based on vector multiplets' real special symmetric scalar manifolds $\frac{\mathrm{SO}(1, n)}{\mathrm{SO}(n)}$, which gives rise to non-symmetric coset manifolds in $D=4$ and in $D=3$.
} 


\begin{tabular}{|c|c|c|}
\hline$D$ & $S O(8) \times S O(D-2+m) \supset H_{D}^{8}(m) \times \mathrm{SO}(D-2)$ & type \\
\hline 10 & $S O(8) \times S O(8+m) \supset S O(m) \times S O(8)$ & $n m, n s$ \\
\hline 9 & $S O(8) \times S O(7+m) \supset S O(m) \times S O(7)$ & $n m, n s$ \\
\hline 8 & $S O(8) \times S O(6+m) \supset S O(2) \times S O(m) \times S O(6)$ & $n m, n s$ \\
\hline 7 & $S O(8) \times S O(5+m) \supset S O(3) \times S O(m) \times S O(5)$ & $n m, n s$ \\
\hline $6, I I A$ & $S O(8) \times S O(4+m) \supset S O(4) \times S O(m) \times \mathrm{SO}(4)$ & $n m, n s$ \\
\hline $6, I I B$ & $S O(8) \times S O(3+m) \supset S O(5) \times S O(m) \times \mathrm{SO}(4)$ & $\max , s$ \\
\hline 5 & $S O(8) \times S O(3+m) \supset S O(5) \times S O(m) \times \mathrm{SO}(3)$ & $n m, n s$ \\
\hline 4 & $S O(8) \times S O(2+m) \supset(\mathrm{SO}(2) \times S O(6) \times S O(m)) \times \mathrm{SO}(2)$ & $\max , s$ \\
\hline
\end{tabular}

Table 4. Embedding $H_{8}^{3} \supset H_{8}^{D} \times \mathrm{SO}(D-2)$ ( 2.1) [23] for half-maximal supergravity theories $(N=8)$ in $10 \geqslant D \geqslant 4$ Lorentzian space-time dimensions. Since matter coupling is allowed, $H_{8}^{3}$ and in general $H_{8}^{D}$ entail both half-maximal $\mathcal{R}$-symmetry and Clifford vacuum symmetry.

For $N=4$, we recall that the Clifford algebra decomposes as

$$
S O(4) \sim \mathrm{SU}(2)_{v} \times \mathrm{SU}(2)_{h},
$$

where $\mathrm{SU}(2)_{v}$ pertains to the $D=3$ reduction of $D=4$ vector multiplets, while $\mathrm{SU}(2)_{h}$ is related to the hypermultiplet sector, which is insensitive to the number of space-time dimensions in which the quarter-maximal theory is defined (namely, $3 \leqslant D \leqslant 6$ ). Since we disregard hypermultiplets, in the treatment below we only consider $\mathrm{SU}(2)_{v}$ (and thus we remove the subscript " $v$ "), which will be a commuting factor in the $m c s$ of the $D=3$ $U$-duality group $G_{4}^{4}$. 


\subsection{Minimal coupling infinite sequence and "pure" $D=4$ supergravity}

We start by considering the infinite sequence of $D=3$ quaternionic Kähler symmetric spaces

$$
\frac{\mathrm{SU}(2,1+n)}{\mathrm{SU}(2) \times \mathrm{SU}(1+n) \times \mathrm{U}(1)},
$$

which can be uplifted only to $D=4$, giving rise to Maxwell-Einstein supergravity models minimally coupled to $n$ vector multiplets [33]. The $D=3 U$-duality group is $G_{4}^{3}=$ $\mathrm{SU}(2,1+n)$.

The embedding of the 4 -dimensional Ehlers group $S L(2, \mathbb{R})$ into $\mathrm{SU}(2, n+1)$ is maximal and symmetric:

$$
\mathrm{SU}(2,1+n) \supset_{s} \mathrm{SL}(2, \mathbb{R}) \times \mathrm{U}(1, n),
$$

and at the mcs level:

$$
\mathrm{SU}(2) \times S U(1+n) \times \mathrm{U}(1) \supset_{s} \mathrm{U}(1)_{J} \times \mathrm{U}(n) \times \mathrm{U}(1)_{\mathcal{R}},
$$

where $D=4 U$-duality group is $G_{4}^{4}=\mathrm{U}(1, n) . \mathrm{U}(1)_{\mathcal{R}}$ in $(4.4)$ is the part of $D=4 \mathcal{R}$ symmetry $\mathrm{U}(2)$ under which the $D=4$ vector multiplets are charged, whereas the $\mathrm{U}(n)$ factor correspond to $D=4$ Clifford vacuum symmetry (completely due to matter coupling).

By merging (4.3) and (4.4), the following $c$-map is obtained [31]:

$$
\mathbb{C P}^{n} \equiv \frac{\mathrm{SU}(1, n)}{\mathrm{U}(n)} \stackrel{c}{\longrightarrow} \frac{\mathrm{SU}(2,1+n)}{\mathrm{SU}(2) \times S U(1+n) \times \mathrm{U}(1)},
$$

where $\mathbb{C P}^{n}$ denotes the complex projective (non-compact) spaces.

Note that for $n=0$ the quaternionic manifold (4.2) is not only Kähler, but also special Kähler, and it is an example of Einstein space with self-dual Weyl curvature (see e.g. [34], and refs. therein). It is usually called the universal hypermultiplet, and it corresponds to the $c$-map of "pure" $\mathcal{N}=2$ supergravity in $D=4$, obtained as " $n=0$ limit" of the $\mathbb{C P}^{n}$ sequence; namely, by specifying $n=0$ in (4.5) [31]:

$$
\varnothing \stackrel{c}{\longrightarrow} \frac{\mathrm{SU}(2,1)}{\mathrm{SU}(2) \times \mathrm{U}(1)} .
$$

Correspondingly, for $n=0$ (4.3) and (4.4) respectively read

$$
\begin{aligned}
\mathrm{SU}(2,1) & \supset_{s} \mathrm{SL}(2, \mathbb{R}) \times \mathrm{U}(1) \sim \mathrm{U}(1,1) ; \\
\mathrm{SU}(2) \times \mathrm{U}(1) & \supset_{s} \mathrm{U}(1)_{J} \times \mathrm{U}(1)_{\mathcal{R}},
\end{aligned}
$$

and thus the 4 bosonic massless states of $\mathcal{N}=2, D=4$ "pure" supergravity are in the $\mathbf{2}_{\mathbb{C}}$ of $\mathrm{SU}(2) \times \mathrm{U}(1) \sim \mathrm{U}(2)=m c s(\mathrm{SU}(2,1))$.

\section{2 "Pure" $D=5,6$ supergravity and $T^{3}$ and $S T^{2}$ models in $D=4$}

\subsection{1 $D=5$}

Within the framework under consideration, "pure" $D=5$ supergravity can be obtained as $D=5$ uplift of the so-called $\mathcal{N}=2, D=4 T^{3}$ model, whose vector multiplet's scalar 
span the symmetric special Kähler manifold $\mathrm{SL}(2, \mathbb{R}) / \mathrm{U}(1)$ (with Ricci scalar curvature $R=-2 / 3[32])$, and whose $D=3 U$-duality group is $G_{4, T^{3}}^{3}=G_{2(2)}$.

The embedding of the 5 -dimensional Ehlers group $S L(3, \mathbb{R})$ into $G_{4, T^{3}}^{3}$ is maximal and non-symmetric (see e.g. [28] and refs. therein):

$$
G_{2(2)} \supset_{n s} \mathrm{SL}(3, \mathbb{R}),
$$

and at the mcs level:

$$
\mathrm{SU}(2) \times \mathrm{SU}(2) \supset_{s} \mathrm{SO}(3)_{J} \sim \mathrm{SU}(2)_{J}
$$

where the $D=5$ massless spin group $\mathrm{SO}(3)_{J}$ is diagonally embedded into $\mathrm{SU}(2) \times \mathrm{SU}(2)=$ $m c s\left(G_{2(2)}\right)$. The 8 bosonic massless states of $\mathcal{N}=2, D=5$ "pure" supergravity are in the $(\mathbf{4}, \mathbf{2})$ of $m c s\left(G_{2(2)}\right)$ itself.

By merging (4.9) and (4.10), the following $c$-map is obtained ${ }^{12}[31]$ :

$$
\left.\frac{\mathrm{SL}(2, \mathbb{R})}{\mathrm{U}(1)}\right|_{T^{3}} \stackrel{c}{\longrightarrow} \frac{G_{2(2)}}{\mathrm{SU}(2) \times \mathrm{SU}(2)}
$$

The corresponding Jordan algebra interpretation of (4.9) reads

$$
Q \operatorname{Conf}(\mathbb{R}) \supset_{s} \mathrm{SL}(3, \mathbb{R}),
$$

because the $T^{3}$ model is related to the (non-generic) simple rank-3 Euclidean Jordan algebra given by the reals $\mathbb{R}$ (see tables $5-8$ ).

\subsection{2 $\quad D=6$}

Analogously, "pure" $D=6(1,0)$ chiral supergravity ${ }^{13}$ can be obtained as $D=6$ uplift of the so-called $\mathcal{N}=2, D=4 S T^{2}$ model, whose vector multiplets' scalars span the symmetric special Kähler manifold $[\mathrm{SL}(2, \mathbb{R}) / \mathrm{U}(1)]^{2}$, and whose $D=3 U$-duality group is $G_{4, S T^{2}}^{3}=\mathrm{SO}(4,3)$.

The embedding of the 6 -dimensional Ehlers group $S L(4, \mathbb{R})$ into $G_{4, S T^{2}}^{3}$ is maximal and symmetric:

$$
\mathrm{SO}(4,3) \supset_{s} S O(3,3) \sim \mathrm{SL}(4, \mathbb{R})
$$

and at the $m c s$ level:

$$
\mathrm{SO}(4) \times \mathrm{SO}(3) \supset_{s} \mathrm{SO}(3) \times \mathrm{SO}(3) \sim \mathrm{SO}(4)_{J}
$$

where the $D=6$ massless spin group is $\mathrm{SO}(4)_{J}$. The 12 bosonic massless states of "pure" $D=6(1,0)$ supergravity are in the $(\mathbf{4}, \mathbf{3})$ of $\mathrm{SO}(4) \times \mathrm{SO}(3)=m c s(S O(4,3))$.

\footnotetext{
${ }^{12}$ Attention should be paid to distinguish $\left.\frac{\mathrm{SL}(2, \mathbb{R})}{\mathrm{U}(1)}\right|_{T^{3}}(R=-2 / 3)$ from the $n=1$ element of the $\mathbb{C P}^{n}$ infinite sequence treated above, namely the $\mathbb{C P}^{1}$ space (axio-dilatonic $\mathcal{N}=2, D=4$ supergravity), which has $R=-2$. Note that $R=-2$ and $R=-2 / 3$ are the unique two values for which the Kähler manifold $\frac{\mathrm{SL}(2, \mathbb{R})}{\mathrm{U}(1)}$ is a special Kähler manifold [32].

${ }^{13}$ We here disregard the various conditions to be fulfilled for anomaly-free chiral supergravity theories in $D=6$ (see e.g. [35-39]).
} 
By merging (4.13) and (4.14), the following c-map is obtained [31]:

$$
\left[\frac{\mathrm{SL}(2, \mathbb{R})}{\mathrm{U}(1)}\right]^{2} \stackrel{c}{\rightarrow} \frac{S O(4,3)}{\mathrm{SO}(4) \times \mathrm{SO}(3)}
$$

The corresponding Jordan algebra interpretation of (4.13) reads

$$
Q \operatorname{Conf}\left(\mathbb{R} \oplus \boldsymbol{\Gamma}_{1,0}\right) \supset_{s} \operatorname{SL}(4, \mathbb{R}),
$$

because the $S T^{2}$ model is related to the (non-generic) semi-simple rank-3 Euclidean Jordan algebra given by $\mathbb{R} \oplus \boldsymbol{\Gamma}_{1,0} \sim \mathbb{R} \oplus \mathbb{R}$.

\subsection{The Jordan symmetric infinite sequence}

The aforementioned $S T^{2}$ model is actually the first element of the so-called Jordan symmetric sequence of quarter-maximal theories.

The $D=3 U$-duality group is $G_{4}^{3}=S O(4, D-2+n)$, where $n$ is the number of matter multiplets in $D=3$ other than those coming from the reduction of the gravity multiplet in $D$ dimensions. Furthermore, $m c s\left(G_{8}^{3}\right)=\mathrm{SO}(4) \times S O(D-2+n)_{C V}$; as mentioned, $\mathrm{SO}(4) \sim \mathrm{SU}(2)_{v} \times \mathrm{SU}(2)_{h}$ is the Clifford algebra for massless particles with $\mathcal{N}=8$ supersymmetries, and $S O(D-2+n)_{C V}$ is the Clifford vacuum symmetry.

Let us consider the relevant chain of maximal embeddings leading to the embedding of the $D$-dimensional Ehlers group ${ }^{14} S L(D-2, \mathbb{R})$ into $S O(4, D-2+n)$.

\subsection{1 $D=6$}

In $D=6$, it suffices to start from $G_{4}^{3}=S O(4,3+n)$, and the corresponding maximal symmetric embedding reads

$$
S O(4,3+n) \supset_{s} S O(3,3) \times S O(1, n) \sim S L(4, \mathbb{R}) \times S O(1, n),
$$

and at the mcs level:

$$
S O(4) \times S O(3+n) \supset_{s} S O(3) \times S O(3) \times \times S O(n) \sim S O(4) \times S O(n),
$$

where $n$ is the number of matter (tensor) multiplets in $D=6$. The group commuting with $S L(4, \mathbb{R})$ inside $S O(4,3+n)$ is nothing but the 6 -dimensional $U$-duality group of tensor multiplets $G_{4}^{6}=\mathrm{SO}(1, n)$.

\subsection{2 $\quad D=5$}

For $D=5$, one branches once more from (4.17), getting:

$$
S O(4,3+n) \supset_{s} S L(4, \mathbb{R}) \times S O(1, n) \supset_{s} S L(3, \mathbb{R}) \times \mathrm{SO}(1,1) \times S O(1, n),
$$

and at the mcs level:

$$
S O(4) \times S O(3+n) \supset_{s} S O(4) \times S O(n) \supset_{s} S O(3) \times S O(n),
$$

where $n+1$ is the number of matter (vector) multiplets in $D=5$. The group commuting with $S L(3, \mathbb{R})$ inside $S O(4,3+n)$ is nothing but the 5 -dimensional $U$-duality group $G_{4}^{5}=$ $\mathrm{SO}(1,1) \times \mathrm{SO}(1, n)$. Note the "enhancement" to $S L(4, \mathbb{R}) \times S O(1, n)$ in (4.19).

\footnotetext{
${ }^{14}$ Note that, consistently, for $n=0$ (in $D=5$ and $D=6$ ) and $n=1$ (in $D=4$ ), one re-obtains the case of the $S T^{2}$ model treated above.
} 


\subsection{3 $D=4$}

For $D=4$, the embedding is maximal and symmetric:

$$
S O(4,2+n) \supset_{s} \mathrm{SO}(2,2) \times \mathrm{SO}(2, n) \sim S L(2, \mathbb{R})_{\text {Ehlers }} \times S L(2, \mathbb{R}) \times \mathrm{SO}(2, n),
$$

and at the mcs level:

$$
S O(4) \times S O(2+n) \supset_{s} S O(2)_{J} \times S O(2) \times S O(2) \times S O(n),
$$

where $n$ is the number of matter (vector) multiplets in $D=4$. The group commuting with $\operatorname{SL}(2, \mathbb{R})_{\text {Ehlers }}$ inside $S O(4,2+n)$ is nothing but the 4-dimensional $U$-duality group $G_{4}^{4}=S L(2, \mathbb{R}) \times \mathrm{SO}(2, n)$. By merging (4.21) and (4.22), one obtains the following $c$-map:

$$
\frac{S L(2, \mathbb{R})}{\mathrm{U}(1)} \times \frac{\mathrm{SO}(2, n)}{\mathrm{SO}(2) \times \mathrm{SO}(n)} \stackrel{c}{\longrightarrow} \frac{\mathrm{SO}(4, n+2)}{\mathrm{SO}(4) \times \mathrm{SO}(n+2)} .
$$

\subsection{Magical models}

Let us now consider the isolated cases of symmetric $N=8$ quarter-maximal theories, the so-called magical models $[19,20]$. They are associated to rank-2 (in $D=6$ ) and rank-3 (in $D=5$ ) Euclidean Jordan algebras over the four normed division algebras $\mathbb{O}$ (octonions), $\mathbb{H}$ (quaternions), $\mathbb{C}$ (complex numbers) and $\mathbb{R}$ (real numbers), and to the Freudenthal triple systems over such algebras (in $D=4$ ). Consequently, they can be parametrized in terms of the real dimension of the relevant division algebra, namely $q=8,4,2,1$ for $\mathbb{O}, \mathbb{H}, \mathbb{C}$ and $\mathbb{R}$, respectively. In this respect, the $T^{3}$ model treated above corresponds to $q=-2 / 3$.

We will now analyze the relevant embeddings in $D=4,5$ and 6 .

\subsection{1 $D=4$}

In $D=4$, the magic models are related to the Freudenthal triple system $\mathfrak{M}\left(J_{3}^{\mathbb{A}}\right)$ over the rank-3 simple Euclidean Jordan algebra $J_{3}^{\mathbb{A}}(\mathbb{A}=\mathbb{O}, \mathbb{H}, \mathbb{C}, \mathbb{R})$. The $D=3$ and $D=4$ $U$-duality groups are nothing but the quasi-conformal and conformal group of $J_{3}^{\mathbb{A}}$, respectively, and they are related by the following maximal symmetric embedding:

$$
G_{4}^{3}(q) \supset_{s} \operatorname{SL}(2, \mathbb{R})_{\text {Ehlers }} \times G_{4}^{4}(q),
$$

with $m c s$ level involving the $D=4$ massless spin group:

$$
m c s\left(G_{4}^{3}(q)\right) \supset_{s} \mathrm{SO}(2)_{J} \times m c s\left(G_{4}^{4}(q)\right) .
$$

(4.24)-(4.25) correspond to the following $c^{*}$-map symmetric embedding of the corresponding scalar manifolds in $D=3$ (para-quaternionic pseudo-Riemannian) and $D=4$ (special Kähler):

$$
\frac{G_{4}^{4}(q)}{m c s\left(G_{4}^{4}(q)\right)} \stackrel{c^{*}}{\rightarrow} \frac{G_{4}^{3}(q)}{\mathrm{SL}(2, \mathbb{R}) \times G_{4}^{4}(q)}
$$

The various cases are listed in tables 5 and 6 . 


\begin{tabular}{|c||c|c|}
\hline $\mathfrak{M}\left(J_{3}^{\mathbb{A}}\right)$ & $G_{4}^{3}(q) \supset_{s} G_{4}^{4}(q) \times S L(2, \mathbb{R})$ & type \\
\hline \hline $\mathfrak{M}\left(J_{3}^{\mathbb{Q}}\right)(q=8)$ & $E_{8(-24)} \supset E_{7(-25)} \times S L(2, \mathbb{R})$ & $\max , s$ \\
\hline $\mathfrak{M}\left(J_{3}^{\mathbb{H}}\right)(q=4)$ & $E_{7(-5)} \supset S O^{*}(12) \times S L(2, \mathbb{R})$ & $\max , s$ \\
\hline $\mathfrak{M}\left(J_{3}^{\mathbb{C}}\right)(q=2)$ & $E_{6(2)} \supset S U(3,3) \times S L(2, \mathbb{R})$ & $\max , s$ \\
\hline $\mathfrak{M}\left(J_{3}^{\mathbb{R}}\right)(q=1)$ & $F_{4(4)} \supset S p(6, \mathbb{R}) \times S L(2, \mathbb{R})$ & $\max , s$ \\
\hline $\mathfrak{M}(\mathbb{R})(q=-2 / 3)$ & $G_{2(2)} \supset S L(2, \mathbb{R}) \times \mathrm{SL}(2, \mathbb{R})$ & $\max , s$ \\
\hline
\end{tabular}

Table 5. Embedding $G_{4}^{3}(q) \supset G_{4}^{4}(q) \times{ }_{s} \mathrm{SL}(2, \mathbb{R})$ Ehlers for magical Maxwell-Einstein supergravity theories $(N=8)$ in $D=4$ Lorentzian space-time dimensions. Also the case of $T^{3}$ model $(q=-2 / 3)$ is reported.

\subsection{2 $D=5$}

In $D=5$, the magic models are related to $J_{3}^{\mathbb{A}}$ 's themselves. The $D=5 U$-duality group is the reduced structure group of $J_{3}^{\mathbb{A}}$, and the embedding of the $D=5$ Ehlers group $\mathrm{SL}(3, \mathbb{R})$ into the $D=3 U$-duality group is maximal and non-symmetric:

$$
G_{4}^{3}(q) \supset_{n s} \operatorname{SL}(3, \mathbb{R}) \times G_{4}^{5}(q),
$$

with $m c s$ level involving the $D=5$ massless spin group:

$$
m c s\left(G_{4}^{3}(q)\right) \supset_{s} \mathrm{SO}(3)_{J} \times m c s\left(G_{4}^{5}(q)\right) .
$$

The various cases are listed in tables 7 and 8 .

\subsection{3 $D=6$}

In $D=6$, the magic models are related to the rank-2 Jordan algebra $J_{2}^{\mathbb{A}} \sim \boldsymbol{\Gamma}_{1, q+1}$ (where " " here denotes a vector space isomorphism). Namely, the $D=6 U$-duality group is nothing but the reduced structure group of $J_{2}^{\mathbb{A}}$ itself, with the exception of the cases corresponding to $q=4$ and $q=2$, which have a further factor ${ }^{15} \mathcal{A}_{q=2}=\mathrm{SO}(3)$ resp.

\footnotetext{
${ }^{15}$ We note that the non-triviality of the factor group $\mathcal{A}_{q}$ in the $D=6 U$-duality group is related to the reality properties of the spinors within the rank-2 Jordan algebras over the quaternions $\left(J_{2}^{\mathbb{H}} \sim \Gamma_{1,5}\right)$ and
} 


\begin{tabular}{|c||c|c|}
\hline $\mathfrak{M}\left(J_{3}^{\mathbb{A}}\right)$ & $m c s\left(G_{4}^{3}(q)\right) \supset_{s} m c s\left(G_{4}^{4}(q)\right) \times \mathrm{SO}(2)_{J}$ & type \\
\hline \hline $\mathfrak{M}\left(J_{3}^{\mathbb{Q}}\right)(q=8)$ & $E_{7(-133)} \times \mathrm{SU}(2) \supset E_{6(-78)} \times \mathrm{U}(1) \times S O(2)_{J}$ & $\max , s$ \\
\hline $\mathfrak{M}\left(J_{3}^{\mathbb{H}}\right)(q=4)$ & $\mathrm{SO}(12) \times \mathrm{SU}(2) \supset \mathrm{U}(6) \times S O(2)_{J}$ & $\max , s$ \\
\hline $\mathfrak{M}\left(J_{3}^{\mathbb{C}}\right)(q=2)$ & $\mathrm{SU}(6) \times \mathrm{SU}(2) \supset S(U(3) \times \mathrm{U}(3)) \times S O(2)_{J}$ & $\max , s$ \\
\hline $\mathfrak{M}\left(J_{3}^{\mathbb{R}}\right)(q=1)$ & $\mathrm{USp}(6) \times \mathrm{SU}(2) \supset \mathrm{U}(3) \times S O(2)_{J}$ & $\max , s$ \\
\hline $\mathfrak{M}(\mathbb{R})(q=-2 / 3)$ & $\mathrm{SU}(2) \times \mathrm{SU}(2) \supset \mathrm{U}(1) \times \mathrm{SO}(2)_{J}$ & $\max , s$ \\
\hline
\end{tabular}

Table 6. Embedding $m c s\left(G_{4}^{3}(q)\right) \supset_{s} m c s\left(G_{4}^{4}(q)\right) \times \mathrm{SO}(2)_{J}$ for magical Maxwell-Einstein supergravity theories $(N=8)$ in $D=4$ Lorentzian space-time dimensions. Also the case of $T^{3}$ model $(q=-2 / 3)$ is reported.

$\mathcal{A}_{q=1}=\mathrm{SO}(2)$ in the $U$-duality group. The embedding of the $D=6$ Ehlers group $\mathrm{SL}(4, \mathbb{R})$ into the $D=3 U$-duality group is obtained by a two-steps chain of maximal and symmetric embeddings $\left(\mathcal{A}_{q}=I d, \mathrm{SO}(3), \mathrm{SO}(2), I d\right.$ respectively for $\left.q=8,4,2,1\right)$ :

$$
G_{4}^{3}(q) \supset_{s} S O(4, q+4) \times \mathcal{A}_{q} \supset_{s} \mathrm{SL}(4, \mathbb{R}) \times \mathrm{SO}(1, q+1) \times \mathcal{A}_{q},
$$

with $m c s$ level involving the $D=6$ massless spin group:

$$
m c s\left(G_{4}^{3}(q)\right) \supset_{s} \mathrm{SO}(4)_{J} \times \mathrm{SO}(q+1) \times m c s\left(\mathcal{A}_{q}\right) .
$$

Note the "enhancement" to $S O(4, q+4) \times \mathcal{A}_{q}$ in (4.29). The various cases are listed in tables 9 and 10 .

\section{Cosets with $\chi=0$ and Poincaré duality}

From the previous treatment, a class of non-compact, pseudo-Riemannian homogeneous spaces can be naturally constructed, with general structure:

$$
M_{N}^{D} \equiv \frac{G_{N}^{3}}{G_{N}^{D} \times \mathrm{SL}(D-2, \mathbb{R})},
$$

over the complex numbers $\left(J_{2}^{\mathbb{C}} \sim \boldsymbol{\Gamma}_{1,3}\right)$, which are respectively pseudo-real (quaternionic) and complex (see e.g. table 2 of [40]). 


\begin{tabular}{|c||c|c|}
\hline$J_{3}^{\mathbb{A}}$ & $G_{4}^{3}(q) \supset_{s} G_{4}^{5}(q) \times S L(3, \mathbb{R})$ & type \\
\hline \hline$J_{3}^{\mathbb{Q}}(q=8)$ & $E_{8(-24)} \supset E_{6(-26)} \times S L(3, \mathbb{R})$ & $\max , n s$ \\
\hline$J_{3}^{\mathbb{H}}(q=4)$ & $E_{7(-5)} \supset S U^{*}(6) \times S L(3, \mathbb{R})$ & $\max , n s$ \\
\hline$J_{3}^{\mathbb{C}}(q=2)$ & $E_{6(2)} \supset S L(3, C) \times S L(3, \mathbb{R})$ & $\max , n s$ \\
\hline$J_{3}^{\mathbb{R}}(q=1)$ & $F_{4(4)} \supset S L(3, R) \times S L(3, \mathbb{R})$ & $\max , n s$ \\
\hline $\mathbb{R}(q=-2 / 3)$ & $G_{2(2)} \supset \operatorname{SL}(3, \mathbb{R})$ & $\max , n s$ \\
\hline
\end{tabular}

Table 7. Embedding $G_{4}^{3}(q) \supset G_{4}^{5}(q) \times{ }_{s} \mathrm{SL}(3, \mathbb{R})$ Ehlers for magical Maxwell-Einstein supergravity theories $(N=8)$ in $D=5$ Lorentzian space-time dimensions. The $D=5$ uplift of $T^{3}$ model is "pure" minimal supergravity

determined by the embedding of the direct product of the $D$-dimensional Ehlers group $\operatorname{SL}(D-2, \mathbb{R})$ and of the $D$-dimensional $U$-duality group $G_{N}^{D}$ of a supergravity with $\mathcal{N}=2 N$ supersymmetries into the $U$-duality group of the same theory reduced to $D=3$ (Lorentzian) space-time dimensions. From previous sections, such an embedding can be maximal or non-maximal (namely, next-to-maximal), and symmetric or non-symmetric, but, as mentioned, it always preserves the rank of the group (1.3), as well as the noncompact rank of the $D=3 \operatorname{coset} G_{N}^{3} / H_{N}^{3}(1.4)$.

Interestingly, the cosets $M_{N}^{D}$ 's (5.1) all share the same feature: they have an equal number of compact and non-compact generators, thus implying the their coset character $\chi[14,15]$ to be vanishing:

$$
\chi\left(M_{N}^{D}\right) \equiv n c\left(M_{N}^{D}\right)-c\left(M_{N}^{D}\right)=0 .
$$

This property can also be related to the "mcs counterpart" of the class of cosets (5.1), given by the compact, Riemannian homogeneous spaces with general structure

$$
\widehat{M}_{N}^{D} \equiv \frac{m c s\left(G_{N}^{3}\right)}{m c s\left(G_{N}^{D}\right) \times \mathrm{SO}(D-2)_{J}},
$$

determined by the embedding of the direct product of the $D$-dimensional massless spin group $\mathrm{SO}(D-2)=m c s(\operatorname{SL}(D-2, \mathbb{R}))$ and of $H_{N}^{D}=m c s\left(G_{N}^{D}\right)$ into $H_{N}^{3}=m c s\left(G_{N}^{3}\right)$. As 


\begin{tabular}{|c||c|c|}
\hline$J_{3}^{\mathbb{A}}$ & $m c s\left(G_{4}^{3}(q)\right) \supset_{s} m c s\left(G_{4}^{5}(q)\right) \times S O(3)_{J}$ & type \\
\hline \hline$J_{3}^{\mathbb{Q}}(q=8)$ & $E_{7(-133)} \times \mathrm{SU}(2) \supset F_{4(-52)} \times S O(3)_{J}$ & $\max , n s$ \\
\hline$J_{3}^{\mathbb{H}}(q=4)$ & $\mathrm{SO}(12) \times \mathrm{SU}(2) \supset \mathrm{USp}(6) \times S O(3)_{J}$ & $\max , n s$ \\
\hline$J_{3}^{\mathbb{C}}(q=2)$ & $\mathrm{SU}(6) \times \mathrm{SU}(2) \supset S U(3) \times S O(3)_{J}$ & $\max , n s$ \\
\hline$J_{3}^{\mathbb{R}}(q=1)$ & $\mathrm{USp}(6) \times \mathrm{SU}(2) \supset \mathrm{SU}(2)_{P} \times S O(3)_{J}$ & $\max , n s$ \\
\hline $\mathbb{R}(q=-2 / 3)$ & $\mathrm{SU}(2) \times \mathrm{SU}(2) \supset \mathrm{SO}(3)_{J, D}$ & $\max , n s$ \\
\hline
\end{tabular}

Table 8. Embedding $m c s\left(G_{4}^{3}(q)\right) \supset m c s\left(G_{4}^{5}(q)\right) \times{ }_{s} \mathrm{SO}(3)_{J}$ for magical Maxwell-Einstein supergravity theories $(N=8)$ in $D=5$ Lorentzian space-time dimensions. $\mathrm{SU}(2)_{P}$ denotes the principal $\mathrm{SU}(2)$, whereas the subscript " $D$ " stands for diagonal embedding

the $M_{N}^{D}$ 's (5.1), also the $\widehat{M}_{N}^{D}$ 's (5.3) can be of various types, namely maximal or next-tomaximal, symmetric or non-symmetric.

However, $\widehat{M}_{N}^{D}$ 's (5.3) all share the same property: the number of compact or noncompact generators of $M_{N}^{D}$ 's (5.1) is always equal to the (real) dimension of the corresponding $\widehat{M}_{N}^{D}$ 's themselves. This is a consequence of (5.2) as well as the general formula on the signature of a pseudo-Riemannian coset $G / H$ (see e.g. [15])

$$
\begin{aligned}
c(G / H) & =\operatorname{dim}_{\mathbb{R}}(m c s(G))-\operatorname{dim}_{\mathbb{R}}(m c s(H)) ; \\
n c(G / H) & =\operatorname{dim}_{\mathbb{R}}(G)-\operatorname{dim}_{\mathbb{R}}(H)-c(G / H),
\end{aligned}
$$

from which thus follows that the compact generators of $M_{N}^{D}$ are the very generators of the corresponding $\widehat{M}_{N}^{D}$ :

$$
n c\left(M_{N}^{D}\right)=c\left(M_{N}^{D}\right)=\operatorname{dim}_{\mathbb{R}}\left(\widehat{M}_{N}^{D}\right)
$$

Along this line, further elaboration is possible. Indeed, it generally holds that

$$
\operatorname{dim}_{\mathbb{R}}\left[\frac{G_{N}^{3}}{G_{N}^{D} \times \mathrm{SL}(D-2, \mathbb{R})}\right]=2 \operatorname{dim}_{\mathbb{R}}\left[\frac{H_{N}^{3}}{H_{N}^{D} \times \mathrm{SO}(D-2)}\right] .
$$

A possible interpretation of these results is as follows. In a supergravity theory in $D$ space-time (Lorentzian) dimensions, the number of bosonic massless degrees of freedom other than the scalar and graviton ones is given by the difference between the dimension 


\begin{tabular}{|c||c|c|}
\hline$J_{2}^{\mathbb{A}}$ & $G_{4}^{3}(q) \supset \mathrm{SO}(1, q+1) \times A_{q} \times \mathrm{SL}(4, \mathbb{R})$ & type \\
\hline \hline$J_{2}^{\mathbb{Q}}(q=8)$ & $E_{8(-24)} \supset \mathrm{SO}(1,9) \times S L(4, \mathbb{R})$ & $n m, n s$ \\
\hline$J_{2}^{\mathbb{H}}(q=4)$ & $E_{7(-5)} \supset S O(1,5) \times \mathrm{SO}(3) \times S L(4, \mathbb{R})$ & $n m, n s$ \\
\hline$J_{2}^{\mathbb{C}}(q=2)$ & $E_{6(2)} \supset S O(1,3) \times \mathrm{SO}(2) \times S L(4, \mathbb{R})$ & $n m, n s$ \\
\hline$J_{2}^{\mathbb{R}}(q=1)$ & $F_{4(4)} \supset S O(1,2) \times S L(4, \mathbb{R})$ & $n m, n s$ \\
\hline
\end{tabular}

Table 9. Embedding $G_{4}^{3}(q) \supset_{n s} G_{4}^{6}(q) \times \mathrm{SL}(4, \mathbb{R})_{\text {Ehlers }}\left(G_{4}^{6}(q)=\mathrm{SO}(1, q+1) \times \mathcal{A}_{q}\right)$ for chiral magical Maxwell-Einstein supergravity theories $(N=8)$ in $D=6$ Lorentzian space-time dimensions. Recall $\mathrm{SO}(1,5) \sim S U^{*}(4), \mathrm{SO}(1,3) \sim S L(3, \mathbb{C}), \mathrm{SO}(1,2) \sim \mathrm{SL}(2, \mathbb{R})$.

\begin{tabular}{|c||c|c|}
\hline$J_{2}^{\mathbb{A}}$ & $m c s\left(G_{4}^{3}(q)\right) \supset m c s\left(G_{4}^{6}(q)\right) \times \mathrm{SO}(4)_{J}$ & type \\
\hline \hline$J_{2}^{\mathbb{Q}}(q=8)$ & $E_{7(-133)} \times \mathrm{SU}(2) \supset \mathrm{SO}(9) \times S O(4)_{J}$ & $n m, n s$ \\
\hline$J_{2}^{\mathbb{H}}(q=4)$ & $\mathrm{SO}(12) \times \mathrm{SU}(2) \supset S O(5) \times \mathrm{SO}(3) \times S O(4)_{J}$ & $n m, n s$ \\
\hline$J_{2}^{\mathbb{C}}(q=2)$ & $\mathrm{SU}(6) \times \mathrm{SU}(2) \supset S O(3) \times \mathrm{SO}(2) \times S O(4)_{J}$ & $n m, n s$ \\
\hline$J_{2}^{\mathbb{R}}(q=1)$ & $\mathrm{USp}(6) \times \mathrm{SU}(2) \supset S O(2) \times S O(4)_{J}$ & $n m, n s$ \\
\hline
\end{tabular}

Table 10. Embedding $m c s\left(G_{4}^{3}(q)\right) \supset_{n s} m c s\left(G_{4}^{5}(q)\right) \times \mathrm{SO}(4)_{J}$ for chiral magical Maxwell-Einstein supergravity theories $(N=8)$ in $D=6$ Lorentzian space-time dimensions.

of the Clifford algebra and the sum of the dimensions of the $D$-dimensional massless spin group and of the $D$-dimensional "Clifford symmetry" (i.e., $\mathcal{R}$-symmetry + Clifford vacuum degeneracy due to matter coupling, if any). 
Section 5.1 lists the cosets $M_{N}^{D}$ 's (5.1) and their " $m c s$ counterparts" $\widehat{M}_{N}^{D}$ 's (5.3) for all $N$ 's and $D$ 's treated in the present investigation. Then, in sections 5.2 and 5.3 an interpretation of the vanishing character (5.2) will be given in terms of Poincaré duality, or equivalently of Hodge involution acting on the cohomology of $M_{N}^{D}$ 's.

\subsection{The cosets}

\subsection{1 $N=16$}

The specification of (5.1) and (5.3) to maximal supergravity $(N=16)$ give rise the following spaces

$$
\begin{aligned}
& M_{16}^{D} \equiv \frac{G_{16}^{3}}{G_{16}^{D} \times \mathrm{SL}(D-2, \mathbb{R})}=\frac{E_{8(8)}}{G_{16}^{D} \times \mathrm{SL}(D-2, \mathbb{R})} \\
& \widehat{M}_{16}^{D} \equiv \frac{H_{16}^{3}}{H_{16}^{D} \times \mathrm{SO}(D-2)}=\frac{\mathrm{SO}(16)}{H_{16}^{D} \times \mathrm{SO}(D-2)}
\end{aligned}
$$

they are listed in table 11, along with their number of compact and non-compact generators. Among $M_{16}^{D}$, s, the unique maximal and symmetric coset is the one pertaining to $D=4$ (cfr. $(2.30))$ :

$$
M_{16}^{4} \equiv \frac{G_{16}^{3}}{G_{16}^{4} \times \mathrm{SL}(2, \mathbb{R})}=\frac{E_{8(8)}}{E_{7(7)} \times \mathrm{SL}(2, \mathbb{R})},
$$

which is a rank-4 para-quaternionic space, as resulting from the classification of [41]. Also the corresponding

$$
\widehat{M}_{16}^{4}=\frac{\mathrm{SO}(16)}{\mathrm{SU}(8) \times \mathrm{SO}(2)}
$$

is a maximal and symmetric space among $\widehat{M}_{16}^{D}$ 's.

\subsection{2 $\quad N=12$}

The specification of (5.1) and (5.3) to supergravity with $N=12$ in $D=5$ and in $D=4$ respectively reads

$$
\begin{aligned}
& M_{12}^{5} \equiv \frac{G_{12}^{3}}{G_{12}^{5} \times \mathrm{SL}(3, \mathbb{R})}=\frac{E_{7(-5)}}{S U^{*}(6) \times \mathrm{SL}(3, \mathbb{R})}, c=n c=45 \\
& \widehat{M}_{12}^{5} \equiv \frac{H_{12}^{3}}{H_{12}^{5} \times \mathrm{SO}(3)_{J}}=\frac{\mathrm{SO}(12) \times \mathrm{SU}(2)}{U S p(6) \times \mathrm{SO}(3)_{J}} ; \\
& M_{12}^{4} \equiv \frac{G_{12}^{3}}{G_{12}^{4} \times \mathrm{SL}(2, \mathbb{R})}=\frac{E_{7(-5)}}{S O^{*}(12) \times \mathrm{SL}(2, \mathbb{R})}, c=n c=32 ; \\
& \widehat{M}_{12}^{4} \equiv \frac{H_{12}^{3}}{H_{12}^{4} \times \mathrm{SO}(2)}=\frac{\mathrm{SO}(12) \times \mathrm{SU}(2)}{\mathrm{SU}(6) \times \mathrm{U}(1) \times \mathrm{SO}(2)_{J}} .
\end{aligned}
$$

They all are maximal cosets, but $M_{12}^{5}$ and $\widehat{M}_{12}^{5}$ are non-symmetric, whereas $M_{12}^{4}$ and $\widehat{M}_{12}^{4}$ are symmetric.

The values of $c=n c$ given in (5.11) and (5.13) match the ones of the magical quartermaximal $(N=4)$ theory for $q=4$ (see (5.40) and (5.44), respectively); indeed, these theories share the same bosonic sector, and they are both related to $J_{3}^{\mathbb{H}}$. 


\begin{tabular}{|c|c|c|c|}
\hline$D$ & $M_{16}^{D}$ & $\widehat{M}_{16}^{D}$ & $\begin{array}{l}c\left(M_{16}^{D}\right)= \\
n c\left(M_{16}^{D}\right)\end{array}$ \\
\hline 11 & $\frac{E_{8(8)}}{\operatorname{SL}(9, \mathbb{R})}$ & $\frac{\mathrm{SO}(16)}{\mathrm{SO}(9)}$ & 84 \\
\hline $10, I I A$ & $\frac{E_{8(8)}}{\mathrm{SO}(1,1) \times \mathrm{SL}(8, \mathbb{R})}$ & $\frac{\mathrm{SO}(16)}{\mathrm{SO}(8)}$ & 92 \\
\hline $10, I I B$ & $\frac{E_{8(8)}}{\mathrm{SL}(2, R) \times \mathrm{SL}(8, \mathbb{R})}$ & $\frac{\mathrm{SO}(16)}{\mathrm{SO}(2) \times \mathrm{SO}(8)}$ & 91 \\
\hline 9 & $\frac{E_{8(8)}}{G L(2, R) \times \operatorname{SL}(7, \mathbb{R})}$ & $\frac{\mathrm{SO}(16)}{\mathrm{SO}(2) \times \mathrm{SO}(7)}$ & 98 \\
\hline 8 & $\frac{E_{8(8)}}{(\mathrm{SL}(2, \mathbb{R}) \times \mathrm{SL}(3, \mathbb{R})) \times \mathrm{SL}(6, \mathbb{R})}$ & $\frac{\mathrm{SO}(16)}{(\mathrm{SO}(2) \times \mathrm{SO}(3)) \times \mathrm{SO}(6)}$ & 101 \\
\hline 7 & $\frac{E_{8(8)}}{\operatorname{SL}(5, \mathbb{R}) \times \operatorname{SL}(5, \mathbb{R})}$ & $\frac{\mathrm{SO}(16)}{\mathrm{SO}(5) \times \mathrm{SO}(5)}$ & 100 \\
\hline 6 & $\frac{E_{8(8)}}{\mathrm{SO}(5,5) \times \mathrm{SL}(4, \mathbb{R})}$ & $\frac{\mathrm{SO}(16)}{\mathrm{SO}(5) \times \mathrm{SO}(5) \times \mathrm{SO}(4)}$ & 94 \\
\hline 5 & $\frac{E_{8(8)}}{E_{6(6)} \times \mathrm{SL}(3, \mathbb{R})}$ & $\frac{\mathrm{SO}(16)}{\mathrm{USp}(8) \times \mathrm{SO}(3)}$ & 81 \\
\hline 4 & $\frac{E_{8(8)}}{E_{7(7)} \times \mathrm{SL}(2, \mathbb{R})}$ & $\frac{\mathrm{SO}(16)}{\mathrm{SU}(8) \times \mathrm{SO}(2)}$ & 56 \\
\hline
\end{tabular}

Table 11. Pseudo-Riemannian non-compact $E_{8(8)}$-cosets $M_{16}^{D}$ ( 5.7$)$ and Riemannian compact $\mathrm{SO}(16)$-cosets $\widehat{M}_{16}^{D}$ ( 5.8) of maximal supergravity theories $(N=16)$ in $11 \geqslant D \geqslant 4$ Lorentzian space-time dimensions. The number of compact generators $c$ (equal to the number $n c$ of noncompact generators) of $M_{16}^{D}$ is also listed. All cosets $M_{16}^{D}$ have vanishing character. 


\subsection{3 $N=10$}

The specification of (5.1) and (5.3) to supergravity with $N=10$ in $D=4$ gives rise to the following symmetric spaces

$$
\begin{aligned}
& M_{10}^{5} \equiv \frac{G_{10}^{3}}{G_{10}^{4} \times S L(2, \mathbb{R})}=\frac{E_{6(-14)}}{\mathrm{SU}(5,1) \times S L(2, \mathbb{R})}, c=n c=20 \\
& \widehat{M}_{10}^{4} \equiv \frac{H_{10}^{3}}{H_{10}^{4} \times S O(2)}=\frac{\mathrm{SO}(10) \times \mathrm{U}(1)}{\mathrm{SU}(5) \times \mathrm{U}(1) \times \mathrm{U}(1)_{J}} .
\end{aligned}
$$

$M_{10}^{5}$ is a rank-4 para-quaternionic coset.

\subsection{4 $N=8$}

The specification of (5.1) and (5.3) to half-maximal supergravity $(N=8)$ gives rise to the following spaces

$$
\begin{aligned}
& M_{8}^{D} \equiv \frac{G_{8}^{3}}{G_{8}^{D} \times \mathrm{SL}(D-2, \mathbb{R})}=\frac{S O(8, D-2+m)}{G_{8}^{D} \times \mathrm{SL}(D-2, \mathbb{R})} ; \\
& \widehat{M}_{8}^{D} \equiv \frac{H_{8}^{3}}{H_{8}^{D} \times \mathrm{SO}(D-2)}=\frac{S O(8) \times S O(D-2+m)}{H_{8}^{D} \times \mathrm{SO}(D-2)}
\end{aligned}
$$

they are listed in table 12, along with their number of compact and non-compact generators.

Among $M_{8}^{D}$ 's, the unique maximal and symmetric cosets are the ones pertaining to $D=6$ IIB and $D=4($ cfr. $(3.3))$ :

$$
\begin{aligned}
M_{8}^{6, I I B} & \equiv \frac{G_{8}^{3}}{G_{8}^{6, I I B} \times \mathrm{SL}(2, \mathbb{R})}=\frac{S O(8,3+m)}{S O(5, m) \times \mathrm{SL}(4, \mathbb{R})} \\
M_{8}^{4} & \equiv \frac{G_{8}^{3}}{G_{8}^{4} \times \mathrm{SL}(2, \mathbb{R})}=\frac{S O(8,2+m)}{\mathrm{SL}(2, \mathbb{R}) \times S O(6, m) \times \mathrm{SL}(2, \mathbb{R})} .
\end{aligned}
$$

\subsection{5 $N=6$}

The specification of (5.1) and (5.3) to supergravity with $N=6$ in $D=4$ gives rise to the following symmetric spaces

$$
\begin{aligned}
& M_{6}^{4} \equiv \frac{G_{6}^{3}}{G_{6}^{4} \times \mathrm{SL}(2, \mathbb{R})}=\frac{\mathrm{SU}(4, m+1)}{\mathrm{SU}(3, m) \times \mathrm{SL}(2, \mathbb{R})}, c=n c=2 m+7 ; \\
& \widehat{M}_{6}^{4} \equiv \frac{H_{6}^{3}}{H_{6}^{4} \times \mathrm{SO}(2)}=\frac{\mathrm{SU}(4) \times S U(m+1) \times \mathrm{U}(1)}{\mathrm{U}(3) \times \mathrm{U}(m) \times \mathrm{U}(1)_{J}} .
\end{aligned}
$$

\subsection{6 $\quad N=4$ symmetric}

Minimal Coupling. The specification of (5.1) and (5.3) to minimally coupled MaxwellEinstein supergravity with $N=4$ in $D=4$ gives rise to the following symmetric spaces

$$
\begin{aligned}
& M_{4}^{4} \equiv \frac{G_{4}^{3}}{G_{4}^{4} \times \mathrm{SL}(2, \mathbb{R})}=\frac{\mathrm{SU}(2,1+n)}{\mathrm{U}(1, n) \times \mathrm{SL}(2, \mathbb{R})}, c=n c=2 n+2 ; \\
& \widehat{M}_{4}^{4} \equiv \frac{H_{4}^{3}}{H_{4}^{4} \times \mathrm{SO}(2)}=\frac{\mathrm{SU}(2) \times \mathrm{SU}(1+n) \times \mathrm{U}(1)}{\mathrm{U}(n) \times \mathrm{U}(1) \times \mathrm{U}(1)}
\end{aligned}
$$




\begin{tabular}{|c|c|c|c|}
\hline$D$ & $M_{8}^{D}$ & $\widehat{M}_{8}^{D}$ & $\begin{array}{l}c\left(M_{8}^{D}\right)= \\
n c\left(M_{8}^{D}\right)\end{array}$ \\
\hline 10 & $\frac{S O(8,8+m)}{(\mathrm{SO}(1,1) \times S O(m)) \times S L(8, \mathbb{R})}$ & $\frac{S O(8) \times S O(8+m)}{S O(m) \times S O(8)}$ & $8 m+28$ \\
\hline 9 & $\frac{S O(8,7+m)}{(\mathrm{SO}(1,1) \times S O(1, m)) \times S L(7, \mathbb{R})}$ & $\frac{S O(8) \times S O(7+m)}{S O(m) \times S O(7)}$ & $7 m+28$ \\
\hline 8 & $\frac{S O(8,6+m)}{(\mathrm{SO}(1,1) \times S O(2, m)) \times S L(6, \mathbb{R})}$ & $\frac{S O(8) \times S O(6+m)}{(S O(2) \times S O(m)) \times S O(6)}$ & $6 m+27$ \\
\hline 7 & $\frac{S O(8,5+m)}{(\mathrm{SO}(1,1) \times S O(3, m)) \times S L(5, \mathbb{R})}$ & $\frac{S O(8) \times S O(5+m)}{(S O(3) \times S O(m)) \times S O(5)}$ & $5 m+25$ \\
\hline $6, I I A$ & $\frac{S O(8,4+m)}{(\mathrm{SO}(1,1) \times S O(4, m)) \times \mathrm{SL}(4, \mathbb{R})}$ & $\frac{S O(8) \times S O(4+m)}{(S O(4) \times S O(m)) \times S O(4)}$ & $4 m+22$ \\
\hline $6, I I B$ & $\frac{S O(8,3+m)}{S O(5, m) \times \operatorname{SL}(4, \mathbb{R})}$ & $\frac{S O(8) \times S O(3+m)}{(S O(5) \times S O(m)) \times S O(4)}$ & $3 m+15$ \\
\hline 5 & $\frac{S O(8,3+m)}{(\mathrm{SO}(1,1) \times S O(5, m)) \times \mathrm{SL}(3, \mathbb{R})}$ & $\frac{S O(8) \times S O(3+m)}{(S O(5) \times S O(m)) \times S O(3)}$ & $3 m+18$ \\
\hline 4 & $\frac{S O(8,2+m)}{(\mathrm{SL}(2, \mathbb{R}) \times S O(6, m)) \times \mathrm{SL}(2, \mathbb{R})}$ & $\frac{S O(8) \times S O(2+m)}{(\mathrm{SO}(2) \times S O(6) \times S O(m)) \times S O(2)}$ & $2 m+12$ \\
\hline
\end{tabular}

Table 12. Pseudo-Riemannian non-compact $M_{8}^{D}$ ( 5.17) and Riemannian compact cosets $\widehat{M}_{8}^{D}$ ( 5.18) of half-maximal supergravity theories $(N=8)$ in $10 \geqslant D \geqslant 4$ Lorentzian space-time dimensions. The number of compact generators $c$ (equal to the number $n c$ of non-compact generators) of $M_{8}^{D}$ is also listed. All cosets $M_{8}^{D}$ have vanishing character.

$M_{4}^{4}$ has rank 1 for $n=0$, and rank 2 for $n \geqslant 1$, and it is para-quaternionic. It is nothing but a suitable pseudo-Riemannian form of the manifold (4.2) itself, namely the $c^{*}$-map of the rank-1 symmetric special Kähler maximal coset in $D=4$ :

$$
\mathbb{C P}^{n} \equiv \frac{\mathrm{SU}(1, n)}{\mathrm{U}(n)} \stackrel{c^{*}}{\rightarrow} \frac{\mathrm{SU}(2,1+n)}{\mathrm{U}(1, n) \times \mathrm{SL}(2, \mathbb{R})}
$$


$T^{3}$ Model. The specification of (5.1) and (5.3) to the so-called $T^{3}$ model in $D=4$ gives rise to the following symmetric spaces

$$
\begin{aligned}
& M_{4, T^{3}}^{4} \equiv \frac{G_{4, T^{3}}^{3}}{G_{4, T^{3}}^{4} \times \mathrm{SL}(2, \mathbb{R})}=\frac{G_{2(2)}}{\mathrm{SL}(2, \mathbb{R}) \times \mathrm{SL}(2, \mathbb{R})_{\text {Ehlers }}}, c=n c=4 \\
& \widehat{M}_{4, T^{3}}^{4} \equiv \frac{m c s\left(G_{4, T^{3}}^{3}\right)}{H_{4, T^{3}}^{4} \times \mathrm{SO}(2)}=\frac{\mathrm{SU}(2) \times \mathrm{SU}(2)}{\mathrm{U}(1) \times \mathrm{U}(1)}
\end{aligned}
$$

$M_{4, T^{3}}^{4}$ is rank-2 para-quaternionic. It is nothing but a suitable pseudo-Riemannian form of the manifold in the r.h.s. of (4.11), namely the $c^{*}$-map of the rank-1 symmetric special Kähler maximal coset in $D=4$ :

$$
\left.\frac{\mathrm{SL}(2, \mathbb{R})}{\mathrm{U}(1)}\right|_{T^{3}} \stackrel{c^{*}}{\longrightarrow} \frac{G_{2(2)}}{\mathrm{SL}(2, \mathbb{R}) \times \mathrm{SL}(2, \mathbb{R})} .
$$

$S T^{2}$ Model. The specification of (5.1) and (5.3) to the so-called $S T^{2}$ model in $D=4$ gives rise to the following symmetric spaces

$$
\begin{aligned}
M_{4, S T^{2}}^{4} & \left.\equiv \frac{G_{4, S T^{2}}^{3}}{G_{4, S T^{2}}^{4} \times \mathrm{SL}(2, \mathbb{R})_{\text {Ehlers }}}=\frac{\mathrm{SO}(4,3)}{\mathrm{SL}(2, \mathbb{R}) \times \mathrm{SL}(2, \mathbb{R}) \times \mathrm{SL}(2, \mathbb{R})_{\mathrm{Ehlers}}}, c=n c \neq 5629\right) \\
\widehat{M}_{4, S T^{2}}^{4} & \equiv \frac{m c s\left(G_{4, S T^{2}}^{3}\right)}{H_{4, S T^{2}}^{4} \times \mathrm{SO}(2)}=\frac{\mathrm{SO}(4) \times \mathrm{SO}(3)}{\mathrm{U}(1) \times \mathrm{U}(1) \times \mathrm{U}(1)} .
\end{aligned}
$$

Jordan symmetric sequence. As mentioned above, the $S T^{2}$ model can be regarded as the first element of the so-called Jordan symmetric sequence of quarter-maximal theories. The specification of (5.1) and (5.3) to such a sequence in $D=6, D=5$ and $D=4$ respectively gives rise to the following spaces:

$D=6:$

$$
\begin{aligned}
& M_{4}^{6} \equiv \frac{G_{4}^{3}}{G_{4}^{6} \times \mathrm{SL}(4, \mathbb{R})}=\frac{S O(4,3+n)}{\mathrm{SO}(1, n) \times \mathrm{SL}(4, \mathbb{R})}, c=n c=3 n+3 ; \\
& \widehat{M}_{4}^{6} \equiv \frac{H_{4}^{3}}{H_{4}^{6} \times \mathrm{SO}(4)}=\frac{\mathrm{SO}(4) \times \mathrm{SO}(3+n)}{\mathrm{SO}(3) \times \mathrm{SO}(n) \times \mathrm{SO}(3)} ;
\end{aligned}
$$

$M_{4}^{6}$ and $\widehat{M}_{4}^{6}$ are maximal and symmetric spaces.

$D=5:$

$$
\begin{aligned}
& M_{4}^{5} \equiv \frac{G_{4}^{3}}{G_{4}^{5} \times \mathrm{SL}(3, \mathbb{R})}=\frac{S O(4,3+n)}{\mathrm{SO}(1,1) \times S O(1, n) \times \mathrm{SL}(3, \mathbb{R})}, c=n c=3 n+6 \\
& \widehat{M}_{4}^{5} \equiv \frac{H_{4}^{3}}{H_{4}^{5} \times \mathrm{SO}(3)}=\frac{\mathrm{SO}(4) \times \mathrm{SO}(3+n)}{\mathrm{SO}(n) \times \mathrm{SO}(3)}
\end{aligned}
$$

$M_{4}^{5}$ and $\widehat{M}_{4}^{5}$ are non-maximal and non-symmetric spaces. 
$D=4:$

$M_{4}^{4} \equiv \frac{G_{4}^{3}}{G_{4}^{4} \times \mathrm{SL}(2, \mathbb{R})_{\text {Ehlers }}}=\frac{S O(4,2+n)}{\mathrm{SL}(2, \mathbb{R}) \times \mathrm{SO}(2, n) \times \mathrm{SL}(2, \mathbb{R})_{\text {Ehlers }}}, c=n c=2 n+4 ;$

$\widehat{M}_{4}^{4} \equiv \frac{m c s\left(G_{4}^{3}\right)}{H_{4}^{4} \times \mathrm{SO}(2)}=\frac{\mathrm{SO}(4) \times \mathrm{SO}(2+n)}{\mathrm{U}(1) \times \mathrm{U}(1) \times \mathrm{SO}(n) \times \mathrm{U}(1)}$

$M_{4}^{4}$ and $\widehat{M}_{4}^{4}$ are maximal and symmetric spaces. $M_{4}^{4}$ is para-quaternionic and it has rank 2 in the case $n=0$ and rank 3 for $n \geqslant 1$; it is nothing but a suitable pseudoRiemannian form of the manifold in the r.h.s. of (4.23), namely the $c^{*}$-map of the symmetric special Kähler maximal coset in $D=4$ :

$$
\frac{S L(2, \mathbb{R})}{\mathrm{U}(1)} \times \frac{\mathrm{SO}(2, n)}{\mathrm{SO}(2) \times \mathrm{SO}(n)} \stackrel{c^{*}}{\rightarrow} \frac{S O(4,2+n)}{\mathrm{SL}(2, \mathbb{R}) \times \mathrm{SO}(2, n) \times \mathrm{SL}(2, \mathbb{R})_{\text {Ehlers }}} .
$$

\section{Magical models.}

$D=4:$ The specification of (5.1) and (5.3) to magical models in $D=4$ gives rise to maximal symmetric spaces. Their general structure reads

$$
\begin{aligned}
& M_{4}^{4}(q) \equiv \frac{G_{4}^{3}(q)}{G_{4}^{4}(q) \times \mathrm{SL}(2, \mathbb{R})_{\text {Ehlers }}} ; \\
& \widehat{M}_{4}^{4}(q) \equiv \frac{H_{4}^{3}(q)}{H_{4}^{4}(q) \times \mathrm{SO}(2)},
\end{aligned}
$$

listed in table 13. The number of compact and non-compact generators of $M_{4}^{4}(q)$ can be $q$-parametrized as follows:

$$
c\left(M_{4}^{4}(q)\right)=n c\left(M_{4}^{4}(q)\right)=6 q+8=\operatorname{dim}_{\mathbb{R}}\left(\mathbf{R}\left(G_{4}^{4}(q)\right)\right),
$$

where $\mathbf{R}$ is the symplectic irrep. of the $D=4 U$-duality group $G_{4}^{4}(q)$ in which the Abelian two-form field strengths sit; see subsection 5.2 for further analysis. Thus, the split of the generators of $M_{4}^{4}(q)$ into a signature $(n c, c=n c)$ is consistent with the Ehlers-doublet irrep. $(\mathbf{R}, \mathbf{2})$ of $G_{4}^{4}(q) \times \mathrm{SL}(2, \mathbb{R})_{\text {Ehlers }}$. Moreover, $M_{4}^{4}(q)$ is a rank4 pseudo-quaternionic space, given by the $c^{*}$-map of the corresponding symmetric special Kähler maximal coset in $D=4$ :

$$
\frac{G_{4}^{4}(q)}{m c s\left(G_{4}^{4}(q)\right)} \stackrel{c^{*}}{\longrightarrow} \frac{G_{4}^{3}(q)}{G_{4}^{4}(q) \times \mathrm{SL}(2, \mathbb{R})} .
$$

$D=5$ : The specification of (5.1) and (5.3) to magical models in $D=5$ gives rise to the maximal, non-symmetric spaces listed in table 14. Their general structure reads

$$
\begin{aligned}
& M_{4}^{5}(q) \equiv \frac{G_{4}^{3}(q)}{G_{4}^{5}(q) \times \mathrm{SL}(3, \mathbb{R})} ; \\
& \widehat{M}_{4}^{5}(q) \equiv \frac{H_{4}^{3}(q)}{H_{4}^{5}(q) \times \mathrm{SO}(3)} .
\end{aligned}
$$


The number of compact and non-compact generators of $M_{4}^{5}(q)$ can be $q$-parametrized as follows:

$$
c\left(M_{4}^{5}(q)\right)=n c\left(M_{4}^{5}(q)\right)=9(q+1)=\operatorname{dim}_{\mathbb{R}}(\mathcal{R}, \mathbf{3}),
$$

where $(\mathcal{R}, \mathbf{3})$ is the irrep. of $G_{4}^{5}(q) \times \operatorname{SL}(3, \mathbb{R})_{\text {Ehlers }}$ Thus, the split of the generators of $M_{4}^{5}(q)$ into a signature $(n c, c=n c)$ is consistent with a pair of Jordan-triplet irreps. $(\mathcal{R}, \mathbf{3})$ (see subsection 5.2 for further analysis).

$D=6:$ The specification of (5.1) and (5.3) to magical models in $D=6$ respectively gives rise to the non-maximal, non-symmetric spaces listed in table $15 .{ }^{16}$ Their general structure reads

$$
\begin{aligned}
& M_{4}^{6}(q) \equiv \frac{G_{4}^{3}(q)}{G_{4}^{6}(q) \times \mathrm{SL}(4, \mathbb{R})} ; \\
& \widehat{M}_{4}^{6}(q) \equiv \frac{H_{4}^{3}(q)}{H_{4}^{6}(q) \times \mathrm{SO}(4)},
\end{aligned}
$$

where the $U$-duality group $G_{4}^{6}(q)$ in $D=6$ reads $\mathrm{SO}(1, q+1) \times \mathcal{A}_{q}$. The number of compact and non-compact generators of $M_{4}^{6}(q)$ can be $q$-parametrized as follows:

$$
c\left(M_{4}^{6}(q)\right)=n c\left(M_{4}^{6}(q)\right)=11 q+6 .
$$

The meaning of $11 q+6$ and the covariant split in terms of irreps. of $\mathrm{SO}(1, q+1) \times$ $m c s\left(\mathcal{A}_{q}\right) \times \mathrm{SO}(4)$ will be discussed in subsection 5.2 .

\subsection{Poincaré duality}

We are now going to analyze the signature split of the manifolds $M_{N}^{D}$ (5.1), focussing on the maximal $(N=32)$ and magical quarter-maximal cases $(N=8)$.

Nicely, the split signature of $M_{N}^{D}$ covariantly decomposes under $m c s\left(G_{N}^{D}\right) \times \mathrm{SO}(D-2)_{J}$ into a pair of sets of irreps., which are related by Poincaré duality (alias eletric-magnetic duality). In other words, the signature of the pseudo-Riemannian manifolds $M_{N}^{D}$ 's naturally arrange the spectrum of $p>0$ massless forms of the corresponding supergravity theory into a pair of sets of irreps. of $m c s\left(G_{N}^{D}\right) \times \mathrm{SO}(D-2)_{J}$, which are interchanged under Poincaré duality.

As a consequence, the $\chi=0$ feature of the manifolds $M_{N}^{D}(5.1)$ is actually Poincaréduality-invariant (or, equivalently, electric-magnetic duality-invariant).

\subsection{1 $N=16$}

1. $D=11$ ( $M$-theory): the relevant manifold is maximal non-symmetric:

$$
M_{16}^{11}=\frac{E_{8(8)}}{S L(9, \mathbb{R})}:\left[\begin{array}{lll} 
& c & n c \\
E_{8(8)}: & 120 & 128 \\
\mathrm{SL}(9, \mathbb{R}) & 36 & 44 \\
M_{16}^{11}: & 84 & 84
\end{array}\right] .
$$

\footnotetext{
${ }^{16}$ Note that the results on $c=n c$ for $q=8$ (magical exceptional supergravity) in $D=4,5,6$ match the results holding for maximal supergravity in the same dimensions. This is not surprising, because maximal $(N=16)$ and exceptional $(N=4)$ theories are respectively related to $J_{3}^{\bigotimes_{s}}$ and $J_{3}^{\bigotimes}$, the unique difference given by the split vs. division form of the octonionic algebra $\mathbb{O}$.
} 


\begin{tabular}{|c|c|c|c|}
\hline $\mathfrak{M}\left(J_{3}^{\mathbb{A}}\right)$ & $M_{4}^{4}(q)$ & $\widehat{M}_{4}^{4}(q)$ & $\begin{array}{l}c\left(M_{4}^{4}(q)\right)= \\
n c\left(M_{4}^{4}(q)\right)\end{array}$ \\
\hline $\mathfrak{M}\left(J_{3}^{\mathbb{Q}}\right) \quad(q=8)$ & $\frac{E_{8(-24)}}{E_{7(-25)} \times S L(2, \mathbb{R})}$ & $\frac{E_{7(-133)} \times \mathrm{SU}(2)}{E_{6(-78) \times \mathrm{U}(1) \times S O(2)}}$ & 56 \\
\hline $\mathfrak{M}\left(J_{3}^{\mathbb{H}}\right) \quad(q=4)$ & $\frac{E_{7(-5)}}{S O^{*}(12) \times S L(2, \mathbb{R})}$ & $\frac{\mathrm{SO}(12) \times \mathrm{SU}(2)}{\mathrm{SU}(6) \times \mathrm{U}(1) \times \mathrm{SO}(2)}$ & 32 \\
\hline $\mathfrak{M}\left(J_{3}^{\mathbb{C}}\right) \quad(q=2)$ & $\frac{E_{6(2)}}{S U(3,3) \times S L(2, \mathbb{R})}$ & $\frac{\mathrm{SU}(6) \times \mathrm{SU}(2)}{S(U(3) \times \mathrm{U}(3)) \times \mathrm{SO}(2)}$ & 20 \\
\hline $\mathfrak{M}\left(J_{3}^{\mathbb{R}}\right) \quad(q=1)$ & $\frac{F_{4(4)}}{S p(6, \mathbb{R}) \times S L(2, \mathbb{R})}$ & $\frac{\mathrm{USp}(6) \times \mathrm{SU}(2)}{\mathrm{SU}(3) \times \mathrm{U}(1) \times \mathrm{SO}(2)}$ & 14 \\
\hline $\mathfrak{M}(\mathbb{R}) \quad(q=-2 / 3)$ & $\frac{G_{2(2)}}{S L(2, \mathbb{R}) \times \operatorname{SL}(2, \mathbb{R})}$ & $\frac{\mathrm{SU}(2) \times \mathrm{SU}(2)}{\mathrm{U}(1) \times \mathrm{SO}(2)_{J}}$ & 4 \\
\hline
\end{tabular}

Table 13. Pseudo-Riemannian, non-compact, maximal, para-quaternionic symmetric $\operatorname{cosets} M_{4}^{4}(q)$ ( 5.38) and Riemannian, compact, maximal cosets $\widehat{M}_{4}^{4}(q)$ ( 5.39) of magic quarter-maximal supergravity theories $(N=4)$ in $D=4$ Lorentzian space-time dimensions. Also the $T^{3}$ model $(q=-2 / 3)$ is reported. The number of compact generators $c$ (equal to the number $n c$ of non-compact generators) of $M_{4}^{4}(q)$ is also listed. All cosets $M_{4}^{4}(q)$ have vanishing character.

Such a signature splitting is covariant with respect to $S O(9)=\operatorname{mcs}(S L(9, \mathbb{R}))$ :

$$
\begin{aligned}
& E_{8(8)} \supset_{n s} \mathrm{SL}(9, \mathbb{R}) ; \\
& \mathbf{2 4 8}=\mathbf{8 0}+\mathbf{8 4}+\mathbf{8 4}^{\prime} ; \\
& S l(9, \mathbb{R}) \stackrel{m c s}{\supset} \mathrm{SO}(9) ; \\
& \mathbf{8 4}^{(\prime)}=\mathbf{8 4} .
\end{aligned}
$$

Therefore, the split $(c, n c)=(84,84)$ can be interpreted as the split into two Poincarédual 84's of $\mathrm{SO}(9)$; namely, the 3-form potential (coupled to M2-brane) and its Poincaré dual 6-form potential (coupled to M5-brane):

$$
(c, n c)=(84,84)=\underset{M 2}{\mathbf{8} 4}+\underset{M 5}{\mathbf{8} 4} \text { of } \mathrm{SO}(9) .
$$

2. $D=10$ IIA: the relevant manifold is non-maximal and non-symmetric:

$$
M_{16}^{10 I I A}=\frac{E_{8(8)}}{\mathrm{SO}(1,1) \times S L(8, \mathbb{R})}:\left[\begin{array}{lll} 
& c & n c \\
E_{8(8)}: & 120 & 128 \\
\mathrm{SO}(1,1) \times \mathrm{SL}(8, \mathbb{R}): & 28 & 36 \\
M_{16}^{10} \text { IIA }: & 92 & 92
\end{array}\right] .
$$




\begin{tabular}{|c||c|c|c|}
\hline$J_{3}^{\mathbb{A}}$ & $M_{4}^{5}(q)$ & $\widehat{M}_{4}^{5}(q)$ & $\begin{array}{c}c\left(M_{4}^{5}(q)\right)= \\
n c\left(M_{4}^{5}(q)\right)\end{array}$ \\
\hline \hline$J_{3}^{\mathbb{Q}}(q=8)$ & $\frac{E_{8(-24)}}{E_{6(-26)} \times S L(3, \mathbb{R})}$ & $\frac{E_{7(-133) \times \mathrm{SU}(2)}}{F_{4(-52)} \times \mathrm{SU}(2) \times S O(3)_{J}}$ & 81 \\
\hline$J_{3}^{\mathbb{H}}(q=4)$ & $\frac{E_{7(-5)}}{S U^{*}(6) \times S L(3, \mathbb{R})}$ & $\frac{\mathrm{SO}(12) \times \mathrm{SU}(2)}{\mathrm{USp}(6) \times S O(3)}$ & 45 \\
\hline$J_{3}^{\mathbb{C}}(q=2)$ & $\frac{E_{6(2)}}{S L(3, \mathbb{C}) \times S L(3, \mathbb{R})}$ & $\frac{\mathrm{SU}(6) \times \mathrm{SU}(2)}{S U(3) \times S O(3)}$ & 27 \\
\hline$J_{3}^{\mathbb{R}}(q=1)$ & $\frac{F_{4(4)}}{S L(3, \mathbb{R}) \times S L(3, \mathbb{R})}$ & $\frac{\mathrm{USp}(6) \times \mathrm{SU}(2)}{\mathrm{SU}(2)_{P} \times S O(3)_{J}}$ & 18 \\
\hline $\mathbb{R}(q=-2 / 3)$ & $G_{2(2)} \supset \mathrm{SL}(3, \mathbb{R})$ & $\frac{\mathrm{SU}(2) \times \mathrm{SU}(2)}{\mathrm{SO}(3)_{J, D}}$ & \\
\hline
\end{tabular}

Table 14. Pseudo-Riemannian, non-compact, maximal, non-symmetric cosets $M_{4}^{5}(q)(5.42)$ and Riemannian, compact, maximal cosets $\widehat{M}_{4}^{5}(q)$ ( 5.43) of magic quarter-maximal supergravity theories $(N=4)$ in $D=5$ Lorentzian space-time dimensions. Also the $D=5$ uplift of $T^{3}$ model $(q=-2 / 3)$, namely minimal "pure" supergravity, is reported. The number of compact generators $c$ (equal to the number $n c$ of non-compact generators) of $M_{4}^{5}(q)$ is also listed. All cosets $M_{4}^{5}(q)$ have vanishing character.

Such a signature splitting is covariant with respect to $S O(8)=$ $m c s(\mathrm{SO}(1,1) \times S L(8, \mathbb{R}))$. Indeed, disregarding $\mathrm{SO}(1,1)$ weights, it holds that:

$$
\begin{aligned}
& E_{8(8)} \supset_{n m} \mathrm{SO}(1,1) \times \mathrm{SL}(8, \mathbb{R}) ; \\
& 248=63+1+8+8^{\prime}+28+28^{\prime}+56+56^{\prime} ; \\
& \mathrm{Sl}(8, \mathbb{R}) \stackrel{m c s}{\supset} \mathrm{SO}(8) ; \\
& \mathbf{8}^{(\prime)}, \mathbf{2 8 ^ { ( \prime ) }}, \mathbf{5 6} \mathbf{6}^{(\prime)}=\mathbf{8}_{v}, \mathbf{2 8}, \mathbf{5 6} \boldsymbol{6}_{v} \text {. }
\end{aligned}
$$

Therefore, the split $(c, n c)=(92,92)$ can be interpreted as the split into two sets of Poincaré-dual irreps. of $\mathrm{SO}(8)$; namely, the graviphoton $C_{\mu}^{(1)} \boldsymbol{8}_{v}$, the 2-form $B_{\mu \nu} \mathbf{2 8}$, the 3-form $C_{\mu \nu \rho}^{(3)} \mathbf{5 6}_{v}$ potentials, and their Poincaré duals, namely the 7 -form $\widetilde{C}_{\mu_{1} \ldots \mu_{7}}$, 6-form $\widetilde{B}_{\mu_{1} \ldots \mu_{6}}$ and 5 -form $\widetilde{C}_{\mu_{1} \ldots \mu_{5}}$ potentials:

$$
(c, n c)=(92,92)=\left(\begin{array}{c}
\mathbf{8}_{v} \\
\underset{C^{(1)}}{\mathbf{2 8}} \\
B^{(2)}
\end{array}+\underset{C^{(3)}}{\mathbf{5 6}}\right)+\left(\underset{\widetilde{C}^{(7)}}{\mathbf{8}}+\underset{\widetilde{B}^{(6)}}{\mathbf{2 8}}+\underset{\widetilde{C}^{(5)}}{\mathbf{5 6} \mathbf{6}_{v}}\right) \text { of } \mathrm{SO}(8)
$$




\begin{tabular}{|c||c|c|c|}
\hline$J_{2}^{\mathbb{A}}$ & $M_{4}^{6}(q)$ & $\widehat{M}_{4}^{6}(q)$ & $\begin{array}{c}c\left(M_{4}^{6}(q)\right)= \\
n c\left(M_{4}^{6}(q)\right)\end{array}$ \\
\hline \hline$J_{2}^{\mathbb{Q}}(q=8)$ & $\frac{E_{8(-24)}}{\mathrm{SO}(1,9) \times S L(4, \mathbb{R})}$ & $\frac{E_{7(-133)} \times \mathrm{SU}(2)}{\mathrm{SO}(9) \times S O(4)_{J}}$ & 94 \\
\hline$J_{2}^{\mathbb{H}}(q=4)$ & $\frac{E_{7(-5)}}{S O(1,5) \times \mathrm{SO}(3) \times S L(4, \mathbb{R})}$ & $\frac{\mathrm{SO}(12) \times \mathrm{SU}(2)}{S O(5) \times \mathrm{SO}(3) \times S O(4)_{J}}$ & 50 \\
\hline$J_{2}^{\mathbb{C}}(q=2)$ & $\frac{E_{6(2)}}{S O(1,3) \times \mathrm{SO}(2) \times S L(4, \mathbb{R})}$ & $\frac{\mathrm{SU}(6) \times \mathrm{SU}(2)}{S O(3) \times \mathrm{SO}(2) \times S O(4)} J$ & 28 \\
\hline$J_{2}^{\mathbb{R}}(q=1)$ & $\frac{F_{4(4)}}{S O(1,2) \times S L(4, \mathbb{R})}$ & $\frac{\mathrm{USp}(6) \times \mathrm{SU}(2)}{S O(2) \times S O(4)_{J}}$ & \\
\hline
\end{tabular}

Table 15. Pseudo-Riemannian, non-compact, non-maximal, non-symmetric cosets $M_{4}^{6}(q)(5.45)$ and Riemannian, compact, non-maximal, non-symmetric cosets $\widehat{M}_{4}^{6}(q)(5.46)$ of magic $(1,0)$ chiral supergravity theories $(N=4)$ in $D=6$ Lorentzian space-time dimensions. The number of compact generators $c$ (equal to the number $n c$ of non-compact generators) of $M_{4}^{6}(q)$ is also listed. All cosets $M_{4}^{6}(q)$ have vanishing character.

3. $D=10$ IIB: the relevant manifold is non-maximal and non-symmetric:

$$
M_{16}^{10} I I B=\frac{E_{8(8)}}{\mathrm{SL}(2, \mathbb{R}) \times S L(8, \mathbb{R})}:\left[\begin{array}{lll} 
& c & n c \\
E_{8(8)}: & 120 & 128 \\
\mathrm{SL}(2, \mathbb{R}) \times \mathrm{SL}(8, \mathbb{R}): & 29 & 37 \\
M_{16}^{10} I I B & 91 & 91
\end{array}\right] .
$$

Such a signature splitting is covariant with respect to $S O(8) \times \mathrm{SO}(2)=$ $m c s(S L(8, \mathbb{R}) \times \mathrm{SL}(2, \mathbb{R}))$. Indeed, it holds that:

$$
\begin{aligned}
& E_{8(8)} \supset_{n m} \mathrm{SL}(8, \mathbb{R}) \times \mathrm{SL}(2, \mathbb{R}) ; \\
& \mathbf{2 4 8}=(\mathbf{6 3}, \mathbf{1})+(\mathbf{1}, \mathbf{3})+(\mathbf{7 0}, \mathbf{1})+(\mathbf{2 8}, \mathbf{2})+\left(\mathbf{2 8}^{\prime}, \mathbf{2}\right) \\
& \mathrm{SL}(8, \mathbb{R}) \times \mathrm{SL}(2, \mathbb{R}) \stackrel{m c s}{\supset} \mathrm{SO}(8) \times \mathrm{SO}(2) ; \\
& (\mathbf{8}, \mathbf{1})=\left(\mathbf{8}_{v}, \mathbf{1}\right) \\
& \left(\mathbf{2 8 ^ { ( \prime ) }}, \mathbf{2}\right)=(\mathbf{2 8}, \mathbf{2}) ; \\
& (\mathbf{7 0}, \mathbf{1})=\left(\mathbf{3 5}_{s}, \mathbf{1}\right)+\left(\mathbf{3 5}_{c}, \mathbf{1}\right) .
\end{aligned}
$$

Therefore, the split $(c, n c)=(91,91)$ can be interpreted as the split into two sets of Poincarè-dual irreps. of $\mathrm{SO}(8) \times \mathrm{SO}(2)$; namely, the 2-form $C_{\mu \nu}^{(2)}(\mathbf{2 8}, \mathbf{2})$ and 4form $C_{\mu_{1} \ldots \mu_{4}}^{(4)}\left(\mathbf{3 5}_{s}, \mathbf{1}\right)$ potentials, and their Poincarè duals, namely the 6 -form $\widetilde{C}_{\mu_{1} \ldots \mu_{6}}$ 
$(\mathbf{2 8}, \mathbf{2})$ and the 4 -form $C_{\mu_{1} \ldots \mu_{4}}^{(4)}\left(\mathbf{3 5}_{c}, \mathbf{1}\right)$ potentials:

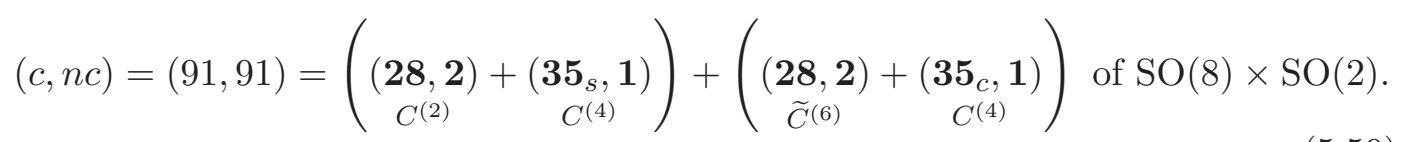

4. $D=9$ : the relevant manifold is non-maximal and non-symmetric:

$$
M_{16}^{9}=\frac{E_{8(8)}}{G L(2, \mathbb{R}) \times S L(7, \mathbb{R})}:\left[\begin{array}{lll} 
& c & n c \\
E_{8(8)}: & 120 & 128 \\
G L(2, \mathbb{R}) \times \mathrm{SL}(7, \mathbb{R}): & 22 & 30 \\
M_{16}^{9}: & 98 & 98
\end{array}\right] .
$$

Such a signature splitting is covariant with respect to $S O(7) \times \mathrm{SO}(2)=$ $m c s(S L(7, \mathbb{R}) \times G L(2, \mathbb{R}))$. Indeed, disregarding $\mathrm{SO}(1,1)$ weights, it holds that:

$$
\begin{aligned}
& E_{8(8)} \supset_{n m} \mathrm{SL}(7, \mathbb{R}) \times G L(2, \mathbb{R}) \\
& \mathbf{2 4 8}=(\mathbf{4 8}, \mathbf{1})+(\mathbf{1}, \mathbf{1})+(\mathbf{1}, \mathbf{3}) \\
& +(\mathbf{7}, \mathbf{1})+\left(\mathbf{7}^{\prime}, \mathbf{1}\right)+(\mathbf{7}, \mathbf{2})+\left(\mathbf{7}^{\prime}, \mathbf{2}\right)+(\mathbf{2 1}, \mathbf{2})+\left(\mathbf{2 1}^{\prime}, \mathbf{2}\right)+(\mathbf{3 5}, \mathbf{1})+\left(\mathbf{3 5}^{\prime}, \mathbf{1}\right) \\
& \mathrm{SL}(7, \mathbb{R}) \stackrel{m c s}{\supset} \mathrm{SO}(7) \\
& \left(\mathbf{7}^{(\prime)}, \mathbf{2} \mathbf{1}^{(\prime)}, \mathbf{3 5}\right)=(\mathbf{7}, \mathbf{2 1}, \mathbf{3 5})
\end{aligned}
$$

Therefore, the split $(c, n c)=(98,98)$ can be interpreted as the split into two sets of Poincarè-dual irreps. of $\mathrm{SO}(7) \times \mathrm{SO}(2)$; namely, the graviphotons $(\mathbf{7}, \mathbf{1})$ and $(\mathbf{7}, \mathbf{2})$, the 2 -form $(\mathbf{2 1}, \mathbf{2})$ and the 3 -form $(\mathbf{3 5}, \mathbf{1})$ potentials, and their Poincarè duals, namely the 6 -forms $(\mathbf{7}, \mathbf{1})$ and $(\mathbf{7}, \mathbf{2})$ duals of graviphotons, the 5 -form $(\mathbf{2 1}, \mathbf{2})$ and the 4 -form $(\mathbf{3 5}, \mathbf{1})$ potentials:

$$
(c, n c)=(98,98)=\left\{\begin{array}{c}
(\mathbf{7}, \mathbf{1})+(\mathbf{7}, \mathbf{2})+(\mathbf{2 1}, \mathbf{2})+(\mathbf{3 5}, \mathbf{1}) \\
+\quad \\
(\mathbf{7}, \mathbf{1})+(\mathbf{7}, \mathbf{2})+(\mathbf{2 1}, \mathbf{2})+(\mathbf{3 5}, \mathbf{1})
\end{array} \text { of } \mathrm{SO}(7) \times \mathrm{SO}(2) .\right.
$$

5. $D=8$ : the relevant manifold is non-maximal and non-symmetric:

$$
M_{16}^{8}=\frac{E_{8(8)}}{\mathrm{SL}(3, \mathbb{R}) \times \mathrm{SL}(2, \mathbb{R}) \times S L(6, \mathbb{R})}:\left[\begin{array}{lll}
c & n c \\
E_{8(8)}: & 120 & 128 \\
\mathrm{SL}(3, \mathbb{R}) \times \mathrm{SL}(2, \mathbb{R}) \times \mathrm{SL}(6, \mathbb{R}): & 19 & 27 \\
M_{16}^{8}: & 101 & 101
\end{array}\right] .
$$

Such a signature splitting is covariant with respect to

$$
S O(6) \times \mathrm{SO}(2) \times \mathrm{SO}(3)=m c s(S L(6, \mathbb{R}) \times \mathrm{SL}(2, \mathbb{R}) \times \mathrm{SL}(3, \mathbb{R})) .
$$


Indeed, it holds that:

$$
\begin{aligned}
& E_{8(8)} \supset_{n m} \mathrm{SL}(6, \mathbb{R}) \times \mathrm{SL}(2, \mathbb{R}) \times \mathrm{SL}(3, \mathbb{R}) \\
& \mathbf{2 4 8}=(\mathbf{3 5}, \mathbf{1}, \mathbf{1})+(\mathbf{1}, \mathbf{3}, \mathbf{1})+(\mathbf{1}, \mathbf{1}, \mathbf{8}) \\
& +(\mathbf{2 0}, \mathbf{2}, \mathbf{1})+\left(\mathbf{6}^{\prime}, \mathbf{2}, \mathbf{3}\right)+\left(\mathbf{6}, \mathbf{2}, \mathbf{3}^{\prime}\right)+(\mathbf{1 5}, \mathbf{1}, \mathbf{3})+\left(\mathbf{1 5}^{\prime}, \mathbf{1}, \mathbf{3}^{\prime}\right) \\
& \mathrm{SL}(6, \mathbb{R}) \times \mathrm{SL}(2, \mathbb{R}) \times \mathrm{SL}(3, \mathbb{R}) \stackrel{\text { mcs }}{\supset} \mathrm{SO}(6) \times \mathrm{SO}(2) \times \mathrm{SO}(3) \\
& \left(\mathbf{6}^{(\prime)}, \mathbf{2}, \mathbf{3}^{(\prime)}\right)=(\mathbf{6}, \mathbf{2}, \mathbf{3}) \\
& \left.(\mathbf{1 5})^{(\prime)}, \mathbf{1}, \mathbf{3}^{(\prime)}\right)=(\mathbf{1 5}, \mathbf{1}, \mathbf{3}) ; \\
& (\mathbf{2 0}, \mathbf{2}, \mathbf{1})=(\mathbf{1 0}, \mathbf{2}, \mathbf{1})+(\overline{\mathbf{1 0}}, \mathbf{2}, \mathbf{1}) .
\end{aligned}
$$

Therefore, the split $(c, n c)=(101,101)$ can be interpreted as the split into two sets of Poincarè-dual irreps. of $\mathrm{SO}(6) \times \mathrm{SO}(2) \times \mathrm{SO}(3)$; namely, the 1 -form $(\mathbf{6}, \mathbf{2}, \mathbf{3})$, the 2 -form $(\mathbf{1 5}, \mathbf{1}, \mathbf{3})$, the 3 -form $(\mathbf{1 0}, \mathbf{2}, \mathbf{1})$ potentials, and their Poincarè duals, namely the 5 -form $(\mathbf{6}, \mathbf{2}, \mathbf{3})$, the 4 -form $(\mathbf{1 5}, \mathbf{1}, \mathbf{3})$ and the 3 -form $(\mathbf{1 0}, \mathbf{2}, \mathbf{1})$ :

$$
\begin{aligned}
(c, n c)= & (101,101) \\
= & \left\{\begin{array}{c}
(\mathbf{6}, \mathbf{2}, \mathbf{3})+(\mathbf{1 5}, \mathbf{1}, \mathbf{3})+(\mathbf{2 1}, \mathbf{2})+(\mathbf{1 0}, \mathbf{2}, \mathbf{1}) \\
+ \\
(\mathbf{6}, \mathbf{2}, \mathbf{3})+(\mathbf{1 5}, \mathbf{1}, \mathbf{3})+(\mathbf{2 1}, \mathbf{2})+(\mathbf{1 0}, \mathbf{2}, \mathbf{1})
\end{array} \text { of } \mathrm{SO}(6) \times \mathrm{SO}(3) \times \mathrm{SO}(2) .\right.
\end{aligned}
$$

6. $D=7$ : the relevant manifold is maximal and non-symmetric:

$$
M_{16}^{7}=\frac{E_{8(8)}}{\operatorname{SL}(5, \mathbb{R}) \times \operatorname{SL}(5, \mathbb{R})}:\left[\begin{array}{lll} 
& c & n c \\
E_{8(8)}: & 120 & 128 \\
\operatorname{SL}(5, \mathbb{R}) \times \operatorname{SL}(5, \mathbb{R}) & 20 & 28 \\
M_{16}^{7}: & 100 & 100
\end{array}\right] .
$$

Such a signature splitting is covariant with respect to $S O(5) \times \mathrm{SO}(5)=$ $m c s(S L(5, \mathbb{R}) \times \mathrm{SL}(5, \mathbb{R}))$. Indeed, it holds that:

$$
\begin{aligned}
& E_{8(8)} \supset_{n s} \mathrm{SL}(5, \mathbb{R}) \times \mathrm{SL}(5, \mathbb{R}) \\
& \mathbf{2 4 8}=(\mathbf{2 4}, \mathbf{1})+(\mathbf{1}, \mathbf{2 4})+(\mathbf{1 0}, \mathbf{5})+\left(\mathbf{1 0}^{\prime}, \mathbf{5}^{\prime}\right)+\left(\mathbf{5}, \mathbf{1 0}^{\prime}\right)+\left(\mathbf{5}^{\prime}, \mathbf{1 0}\right) \\
& \mathrm{SL}(5, \mathbb{R}) \times \mathrm{SL}(5, \mathbb{R}) \stackrel{m c s}{\supset} \mathrm{SO}(5) \times \mathrm{SO}(5) \\
& \left(\mathbf{1 0}^{(\prime)}, \mathbf{5}^{(\prime)}\right)=(\mathbf{1 0}, \mathbf{5})
\end{aligned}
$$

Therefore, the split $(c, n c)=(100,100)$ can be interpreted as the split into two sets of Poincarè-dual irreps. of $\mathrm{SO}(5) \times \mathrm{SO}(5) \sim \mathrm{USp}(4) \times \mathrm{USp}(4)$; namely, the 1-form $(\mathbf{1 0}, \mathbf{5})$ and the 2 -form $(\mathbf{5}, \mathbf{1 0})$ potentials, and their Poincarè duals, namely the 4 -form $(\mathbf{1 0}, \mathbf{5})$ and the 3 -form $(\mathbf{5}, \mathbf{1 0})$ potentials:

$$
(c, n c)=(100,100)=((\mathbf{1 0}, \mathbf{5})+(\mathbf{5}, \mathbf{1 0}))+((\mathbf{1 0}, \mathbf{5})+(\mathbf{5}, \mathbf{1 0})) \text { of } \mathrm{SO}(5) \times \mathrm{SO}(5) .
$$


7. $D=6$ (non chiral $(2,2)$ ): the relevant manifold is non-maximal and non-symmetric:

$$
M_{16}^{6}=\frac{E_{8(8)}}{\mathrm{SO}(5,5) \times \mathrm{SL}(4, \mathbb{R})}:\left[\begin{array}{lll} 
& c & n c \\
E_{8(8)}: & 120 & 128 \\
\mathrm{SO}(5,5) \times \mathrm{SL}(4, \mathbb{R}) & 26 & 34 \\
M_{16}^{6}: & 94 & 94
\end{array}\right] .
$$

Such a signature splitting is covariant with respect to

$$
\begin{aligned}
m c s(S L(5, \mathbb{R}) \times \mathrm{SL}(5, \mathbb{R})) & =S O(5) \times \mathrm{SO}(5) \times \mathrm{SO}(3) \times \mathrm{SO}(3) \\
& \sim \mathrm{USp}(4)_{L} \times \mathrm{USp}(4)_{R} \times \mathrm{SU}(2) \times \mathrm{SU}(2) .
\end{aligned}
$$

Indeed, it holds that:

$$
\begin{aligned}
& E_{8(8)} \supset_{n s} \mathrm{SO}(5,5) \times \mathrm{SL}(4, \mathbb{R}) \\
& \mathbf{2 4 8}=(\mathbf{4 5}, \mathbf{1})+(\mathbf{1}, \mathbf{1 5})+(\mathbf{1 0}, \mathbf{6})+(\mathbf{1 6}, \mathbf{4})+\left(\mathbf{1 6}^{\prime}, \mathbf{4}^{\prime}\right) ; \\
& \mathrm{SO}(5,5) \times \mathrm{SL}(4, \mathbb{R}) \stackrel{m c s}{\supset} \mathrm{USp}(4)_{L} \times \mathrm{USp}(4)_{R} \times \mathrm{SU}(2) \times \mathrm{SU}(2) ; \\
& (\mathbf{1 0 , 6})=(\mathbf{1}, \mathbf{5}, \mathbf{1}, \mathbf{3})+(\mathbf{1}, \mathbf{5}, \mathbf{3}, \mathbf{1})+(\mathbf{5}, \mathbf{1}, \mathbf{1}, \mathbf{3})+(\mathbf{5}, \mathbf{1}, \mathbf{3}, \mathbf{1}) ; \\
& \left(\mathbf{1 6}^{(\prime)}, \mathbf{4}^{(\prime)}\right)=(\mathbf{4}, \mathbf{4}, \mathbf{2}, \mathbf{2}) .
\end{aligned}
$$

Therefore, the split $(c, n c)=(94,94)$ can be interpreted as the split into two sets of Poincarè-dual irreps. of $\mathrm{USp}(4)_{L} \times \mathrm{USp}(4)_{R} \times \mathrm{SU}(2) \times \mathrm{SU}(2)$; namely, the 5 self-dual 2 -forms $B_{\mu \nu \mid R}^{+}(\mathbf{1}, \mathbf{5}, \mathbf{1}, \mathbf{3})$, the 5 anti-self-dual 2-forms $B_{\mu \nu \mid L}^{-}(\mathbf{5}, \mathbf{1}, \mathbf{3}, \mathbf{1})$ and the 16 1-forms $A_{\mu}^{\alpha \dot{\alpha}}(\mathbf{4}, \mathbf{4}, \mathbf{2}, \mathbf{2})$ potentials, and their Poincarè duals, namely the 5 anti-selfdual 2-forms $B_{\mu \nu \mid R}^{-}(\mathbf{1}, \mathbf{5}, \mathbf{3}, \mathbf{1})$, the 5 self-dual 2 -form $B_{\mu \nu \mid L}^{+}(\mathbf{5}, \mathbf{1}, \mathbf{1}, \mathbf{3})$ and the 16 3 -form $\widetilde{A}_{\mu_{1} \ldots \mu_{4}}^{\alpha \dot{\alpha}}(\mathbf{4}, \mathbf{4}, \mathbf{2}, \mathbf{2})$ potentials:

$(c, n c)=(94,94)$

$$
=\left\{\begin{array}{c}
(\mathbf{1}, \mathbf{5}, \mathbf{1}, \mathbf{3})+(\mathbf{5}, \mathbf{1}, \mathbf{3}, \mathbf{1})+(\mathbf{4}, \mathbf{4}, \mathbf{2}, \mathbf{2}) \\
+ \\
(\mathbf{1}, \mathbf{5}, \mathbf{3}, \mathbf{1})+(\mathbf{5}, \mathbf{1}, \mathbf{1}, \mathbf{3})+(\mathbf{4}, \mathbf{4}, \mathbf{2}, \mathbf{2})
\end{array} \text { of } \mathrm{USp}(4)_{L} \times \mathrm{USp}(4)_{R} \times \mathrm{SU}(2) \times \mathrm{SU}(2) .\right.
$$

8. $D=5$ : the relevant manifold is maximal and non-symmetric:

$$
M_{16}^{5}=\frac{E_{8(8)}}{E_{6(6)} \times \mathrm{SL}(3, \mathbb{R})}:\left[\begin{array}{lll} 
& c & n c \\
E_{8(8)}: & 120 & 128 \\
E_{6(6)} \times \mathrm{SL}(3, \mathbb{R}) & 39 & 47 \\
M_{16}^{5}: & 81 & 81
\end{array}\right] .
$$

Such a signature splitting is covariant with respect to $\operatorname{USp}(8) \times \mathrm{SO}(3)=$ $m c s\left(E_{6(6)} \times \mathrm{SL}(3, \mathbb{R})\right)$. Indeed, it holds that:

$$
\begin{aligned}
& E_{8(8)} \supset_{n s} \mathrm{SL}(3, \mathbb{R}) \times E_{6(6)} \\
& \mathbf{2 4 8}=(\mathbf{8}, \mathbf{1})+(\mathbf{1}, \mathbf{7 8})+(\mathbf{3}, \mathbf{2 7})+\left(\mathbf{3}^{\prime}, \mathbf{2 7 ^ { \prime }}\right) \\
& \mathrm{SL}(3, \mathbb{R}) \times E_{6(6)} \stackrel{m c s}{\supset} \mathrm{SO}(3) \times \operatorname{USp}(8) \\
& \left(\mathbf{3}^{(\prime)}, \mathbf{2 7}^{(\prime)}\right)=(\mathbf{3}, \mathbf{2 7}) .
\end{aligned}
$$


Therefore, the split $(c, n c)=(81,81)$, which is related to the so-called Jordan pairs (see e.g. [22]), can be interpreted as the split into two sets of Poincarè-dual irreps. of $\mathrm{SO}(3) \times \mathrm{USp}(8)$; namely, the 27 graviphotons $A_{\mu}(\mathbf{3}, \mathbf{2 7})$, and their Poincarè duals, namely the 27 -forms $\widetilde{A}_{\mu \nu}(\mathbf{3}, \mathbf{2 7})$ :

$$
(c, n c)=(81,81)=(\mathbf{3}, \mathbf{2 7})+(\mathbf{3}, \mathbf{2 7}) \text { of } \mathrm{SO}(3) \times \mathrm{USp}(8) .
$$

Note that the 3 of the massless spin group $\mathrm{SO}(3) \equiv \mathrm{SO}(3)_{J}$ corresponds to the three physical polarizations of the graviphotons in $D=5$.

9. $D=4$ : the relevant manifold is para-quaternionic, maximal and symmetric:

$$
M_{16}^{4}=\frac{E_{8(8)}}{E_{7(7)} \times \mathrm{SL}(2, \mathbb{R})}:\left[\begin{array}{lll} 
& c & n c \\
E_{8(8)}: & 120 & 128 \\
E_{7(7)} \times \mathrm{SL}(2, \mathbb{R}) & 64 & 72 \\
M_{16}^{4}: & 56 & 56
\end{array}\right] .
$$

Such a signature splitting is covariant with respect to $\mathrm{SU}(8) \times \mathrm{SO}(2)_{J}=$ $m c s\left(E_{7(7)} \times \mathrm{SL}(2, \mathbb{R})\right)$. Indeed, it holds that:

$$
\begin{aligned}
& E_{8(8)} \supset_{n s} \mathrm{SL}(2, \mathbb{R}) \times E_{7(7)} ; \\
& \mathbf{2 4 8}=(\mathbf{3}, \mathbf{1})+(\mathbf{1}, \mathbf{1 3 3})+(\mathbf{2}, \mathbf{5 6}) ; \\
& \mathrm{SL}(2, \mathbb{R}) \times E_{7(7)} \stackrel{m c s}{\supset} \mathrm{SO}(2)_{J} \times \mathrm{SU}(8) ; \\
& (\mathbf{2}, \mathbf{5 6})=(\mathbf{2}, \mathbf{2 8})+(\mathbf{2}, \overline{\mathbf{2 8}}) .
\end{aligned}
$$

Therefore, the split $(c, n c)=(56,56)$, which corresponds to a pair of Freudenthal systems $\mathfrak{M}\left(J_{3}^{\mathbb{O}_{s}}\right)$, can be interpreted as the split into two sets of Poincarè-dual irreps. of $\mathrm{SO}(2)_{J} \times \mathrm{SU}(8)$; namely, the 28 graviphotons $A_{\mu}(\mathbf{2}, \mathbf{2 8})$, and their Poincarè-Hodge duals, namely the 28 graviphotons $\widetilde{A}_{\mu}(\mathbf{2}, \overline{\mathbf{2 8}})$ :

$$
(c, n c)=(56,56)=(\mathbf{2}, \mathbf{2 8})+(\mathbf{2}, \overline{\mathbf{2 8}}) \text { of } \mathrm{SO}(2)_{J} \times \mathrm{SU}(8) .
$$

Note that the $\mathbf{2}$ of the massless spin group $\mathrm{SO}(2)_{J}$ corresponds to the two physical polarizations of the graviphotons in $D=4$.

\subsection{2 $N=4$ Magical models}

$D=4$ : the relevant manifold is para-quaternionic, maximal and symmetric (recall (5.38) and (5.40)):

$$
M_{4}^{4}(q) \equiv \frac{G_{4}^{3}(q)}{G_{4}^{4}(q) \times \mathrm{SL}(2, \mathbb{R})_{\text {Ehlers }}}:(c, n c)=(6 q+8,6 q+8) .
$$

Such a signature splitting is covariant with respect to $m c s\left(G_{4}^{4}(q)\right) \times \mathrm{SO}(2)_{J}$. Indeed, it holds that:

$$
\begin{aligned}
& G_{4}^{3}(q) \supset_{s} \operatorname{SL}(2, \mathbb{R}) \times G_{4}^{4}(q) ; \\
& \mathbf{A d j}_{G_{4}^{3}}=(\mathbf{3}, \mathbf{1})+\left(\mathbf{1}, \mathbf{A d j}_{G_{4}^{4}}\right)+(\mathbf{2}, \mathbf{R}) ; \\
& \mathrm{SL}(2, \mathbb{R}) \times G_{4}^{4} \stackrel{m c s}{\supset} \mathrm{SO}(2)_{J} \times m c s\left(G_{4}^{4}\right) ; \\
& (\mathbf{2}, \mathbf{R})=(\mathbf{2}, \mathbf{1})+(\mathbf{2}, \mathcal{R})+(\mathbf{2}, \mathbf{1})+(\mathbf{2}, \overline{\mathcal{R}}),
\end{aligned}
$$


where the bar here denotes the conjugate irrep. $\mathbf{R}(\operatorname{dim}=6 q+8)$ denotes the relevant symplectic irrep. of $G_{4}^{4}$ into which the vectors sit, and $\mathcal{R}(\overline{\mathcal{R}})$ is its electric (magnetic) $D=5$ counterpart, of dimension $3 q+3$. The irrep. $\mathbf{R}$ is given by: ${ }^{17}$

$$
\begin{array}{cccccc}
q: & 8 & 4 & 2 & 1 & -2 / 3 \\
G_{4}^{4}: & E_{7(-25)} & S O^{*}(12) & \mathrm{SU}(3,3) & \mathrm{Sp}(6, \mathbb{R}) & \mathrm{SL}(2, \mathbb{R}) \\
\mathbf{R}: & \mathbf{5 6} & \mathbf{3 2}^{\prime} & \mathbf{2 0} & \mathbf{1 4}^{\prime} & \mathbf{4}
\end{array}
$$

On the other hand, the irrep. $\mathcal{R}$ is given by:

$$
\begin{array}{cccccc}
q: & 8 & 4 & 2 & 1 & -2 / 3 \\
G_{4}^{5}: & E_{6(-26)} & S U^{*}(6) & \mathrm{SL}(3, \mathbb{C}) & \mathrm{SL}(3, \mathbb{R}) & \mathrm{SL}(2, \mathbb{R}) \\
\mathcal{R}: & \mathbf{2 7} & \mathbf{1 5} & \mathbf{9}=(\mathbf{3}, \overline{\mathbf{3}}) & \mathbf{6}^{\prime} & \mathbf{1}
\end{array}
$$

Therefore, the split of signature of $M_{4}^{4}(q)$, which corresponds to a pair of Freudenthal systems $\mathfrak{M}\left(J_{3}^{\mathbb{A}}\right)$, can be interpreted as the split into two sets of Poincarè-dual irreps. of $\mathrm{SO}(2)_{J} \times m c s\left(G_{4}^{4}\right)$; namely, the $D=4$ graviphoton $A_{\mu}(\mathbf{2}, \mathbf{1})$ and the $3 q+3$ matter vectors $(\mathbf{2}, \mathcal{R})$, and their Poincarè duals, namely the graviphoton $A_{\mu}(\mathbf{2}, \mathbf{1})$ and the $3 q+3$ matter vectors $(\mathbf{2}, \overline{\mathcal{R}}):$

$$
(c, n c)=(6 q+8,6 q+8)=((\mathbf{2}, \mathbf{1})+(\mathbf{2}, \mathcal{R}))+((\mathbf{2}, \mathbf{1})+(\mathbf{2}, \overline{\mathcal{R}})) \text { of } \mathrm{SO}(2)_{J} \times m c s\left(G_{4}^{4}\right) .
$$

Note that the $\mathbf{2}$ of the massless spin group $\mathrm{SO}(2)_{J}$ corresponds to the two physical polarizations of the graviphotons.

$D=5$ : the relevant manifold is para-quaternionic, maximal and non-symmetric (recall (5.42) and (5.44)):

$$
M_{4}^{5}(q) \equiv \frac{G_{4}^{3}(q)}{G_{4}^{5}(q) \times \mathrm{SL}(3, \mathbb{R})}:(c, n c)=(9(q+1), 9(q+1)) .
$$

Such a signature splitting is covariant with respect to $m c s\left(G_{4}^{5}\right) \times \mathrm{SO}(3)$. Indeed, it holds that:

$$
\begin{aligned}
& G_{4}^{3}(q) \supset_{s} \mathrm{SL}(3, \mathbb{R}) \times G_{4}^{5}(q) \\
& \mathbf{A d j}_{G_{4}^{3}}=(\mathbf{8}, \mathbf{1})+\left(\mathbf{1}, \mathbf{A d j}_{G_{4}^{5}}\right)+(\mathbf{3}, \mathcal{R})+\left(\mathbf{3}^{\prime}, \mathcal{R}^{\prime}\right) \\
& \mathrm{SL}(3, \mathbb{R}) \times G_{4}^{5}(q) \stackrel{m c s}{\supset} \mathrm{SO}(3) \times m c s\left(G_{4}^{5}\right) \\
& \left(\mathbf{3}^{(\prime)}, \mathcal{R}^{(\prime)}\right)=(\mathbf{3}, \mathbf{1})+(\mathbf{3}, \mathfrak{R}),
\end{aligned}
$$

where the prime here denotes the non-compact analogue of conjugation. $\mathfrak{R}$ (dim= $3 q+2)$ denotes the relevant irrep. of $m c s\left(G_{4}^{5}\right)$ into which the $D=5$ matter vectors sit. Therefore, the split of signature of $M_{4}^{5}(q)$, which corresponds to a Jordan pair (see e.g. [22]), can be interpreted as the split into two sets of Poincarè-dual irreps. of $\mathrm{SO}(3)_{J} \times m c s\left(G_{4}^{5}\right)$; namely, the $D=5$ graviphoton $A_{\mu}(\mathbf{3}, \mathbf{1})$ and the $3 q+2$ matter

\footnotetext{
${ }^{17}$ Actually, in the case $q=4, \mathbf{3 2}^{\prime}$ is the conjugate of the irreps. 32 in which the vectors sit; see appendix B for further detail.
} 
vectors $(\mathbf{3}, \mathfrak{R})$, and their Poincarè duals, namely the graviphoton $A_{\mu}(\mathbf{3}, \mathbf{1})$ and the $3 q+2$ matter vectors $(\mathbf{2}, \mathfrak{R})$ :

$$
\begin{aligned}
(c, n c) & =(9(q+1), 9(q+1)) \\
& =((\mathbf{3}, \mathbf{1})+(\mathbf{3}, \mathfrak{R}))+((\mathbf{3}, \mathbf{1})+(\mathbf{3}, \mathfrak{R})) \text { of } \mathrm{SO}(3) \times m c s\left(G_{4}^{5}\right) .
\end{aligned}
$$

Note that the 3 of the massless spin group $\mathrm{SO}(3) \equiv \mathrm{SO}(3)_{J}$ corresponds to the three physical polarizations of the vectors in $D=5$.

$D=6$ : the relevant manifold is maximal and non-symmetric (recall (5.45) and (5.47)):

$$
M_{4}^{6}(q) \equiv \frac{G_{4}^{3}(q)}{S O(1, q+1) \times \mathcal{A}_{q} \times \operatorname{SL}(4, \mathbb{R})}:(c, n c)=(11 q+6,11 q+6) .
$$

Such a signature splitting is covariant with respect to $S O(q+1) \times m c s\left(\mathcal{A}_{q}\right) \times \mathrm{SO}(4)$. Indeed, it holds that:

$$
\begin{aligned}
& G_{4}^{3}(q) \supset_{s} \operatorname{SL}(4, \mathbb{R}) \times S O(1, q+1) \times \mathcal{A}_{q} \\
& \mathbf{A d j}_{G_{4}^{3}}=(\mathbf{1 5}, \mathbf{1}, \mathbf{1})+\left(\mathbf{1}, \mathbf{A d j}_{S O(1, q+1)}, \mathbf{1}\right)+\left(\mathbf{1}, \mathbf{1}, \mathbf{A d j}_{\mathcal{A}_{q}}\right) \\
& +(\mathbf{4}, \mathbf{S p i n}, \mathbf{2})+\left(\mathbf{4}^{\prime}, \mathbf{S p i n}^{\prime}, \mathbf{2}\right)+(\mathbf{6}, \mathbf{q}+\mathbf{2}, \mathbf{1}) \\
& \mathrm{SL}(4, \mathbb{R}) \times S O(1, q+1) \times \mathcal{A}_{q} \stackrel{m c s}{\supset} \mathrm{SU}(2) \times \operatorname{SU}(2) \times S O(q+1) \times m c s\left(\mathcal{A}_{q}\right) \\
& \left(\mathbf{4}^{(\prime)}, \operatorname{Spin}^{(\prime)}, \mathbf{2}\right)=(\mathbf{2}, \mathbf{2}, \mathbf{S p i n}, \mathbf{2}) \\
& (\mathbf{6}, \mathbf{q}+\mathbf{2}, \mathbf{1})=(\mathbf{3}, \mathbf{1}, \mathbf{q}+\mathbf{1}, \mathbf{1})+(\mathbf{1}, \mathbf{3}, \mathbf{q}+\mathbf{1}, \mathbf{1})+(\mathbf{3}, \mathbf{1}, \mathbf{1}, \mathbf{1})+(\mathbf{1}, \mathbf{3}, \mathbf{1}, \mathbf{1})
\end{aligned}
$$

In (5.97), Spin, $\mathbf{S p i n}^{\prime}$ and $\mathbf{q}+\mathbf{2}$ respectively denote the two conjugate chiral (semi)spinors and the vector irreps. of $S O(1, q+1) \sim \mathrm{SL}(2, \mathbb{A})$ (with $\mathbb{A}=\mathbb{R}, \mathbb{C}, \mathbb{H}, \mathbb{O}$ for $q=1,2,4,8$, respectively), whereas in the right-hand side of (5.98) Spin and $\mathbf{q}+1$ respectively denote the spinor and vector irreps. of $S O(q+1)$. The irrep. Spin of $S O(q+1)$ is given by:

$$
\begin{aligned}
& \begin{array}{cccccc}
q: & 8 & 4 & 2 & 1
\end{array} \\
& \mathrm{SO}(q+1): \mathrm{SO}(9) \mathrm{SO}(5) \mathrm{SO}(3) \mathrm{SO}(2) \\
& \begin{array}{lllll}
\text { Spin : } & 16 & 4 & 2 & 2
\end{array}
\end{aligned}
$$

Thus, through these branchings, the resulting pair of Poincarè-dual irreps. of $\mathrm{SU}(2) \times \mathrm{SU}(2) \times S O(q+1) \times m c s\left(\mathcal{A}_{q}\right)$ irreps. is composed by: $\left.i\right)$ the physical polarizations $(\mathbf{2}, \mathbf{2}$, Spin, 2$)$ of massless 1 -forms and the physical polarizations of 2 forms, which (under the assumption of gravity sector to be anti-self-dual) split into $(\mathbf{1}, \mathbf{3}, \mathbf{1}, \mathbf{1})$ (anti-self-dual gravity sector) and $(\mathbf{3}, \mathbf{1}, \mathbf{q}+\mathbf{1}, \mathbf{1})$ (self-dual matter sector); ii) the physical polarizations $(\mathbf{2}, \mathbf{2}$, Spin, 2 ) of massless 3 -forms and the physical polarizations of Poincaré-dual 2-forms, which split into $(\mathbf{3}, \mathbf{1}, \mathbf{1}, \mathbf{1})$ (self-dual Poincarédual gravity sector) and $(\mathbf{1}, \mathbf{3}, \mathbf{q}+\mathbf{1}, \mathbf{1})$ (anti-self-dual Poincaré-dual matter sector). The real dimension of each set of such irreps. can be computed as (here square brackets denote the integer part)

$$
2^{[q / 2]+2+\left(1-\delta_{q, 8}\right)}+3(q+2)=11 q+6,
$$


and thus corresponds to the signature split of $M_{4}^{6}(q)$ in terms of irreps. of $\mathrm{SU}(2) \times$ $\mathrm{SU}(2) \times S O(q+1) \times m c s\left(\mathcal{A}_{q}\right):$

$$
(c, n c)=(11 q+6,11 q+6)=\left\{\begin{array}{c}
(\mathbf{2}, \mathbf{2}, \mathbf{S p i n}, \mathbf{2})+(\mathbf{1}, \mathbf{3}, \mathbf{1}, \mathbf{1})+(\mathbf{3}, \mathbf{1}, \mathbf{q}+\mathbf{1}, \mathbf{1}) \\
+ \\
(\mathbf{2}, \mathbf{2}, \mathbf{S p i n}, \mathbf{2})+(\mathbf{3}, \mathbf{1}, \mathbf{1}, \mathbf{1})+(\mathbf{1}, \mathbf{3}, \mathbf{q}+\mathbf{1}, \mathbf{1}) .
\end{array}\right.
$$

\subsection{Hodge involution and coset cohomology}

A general property of the $\operatorname{cosets} M_{N}^{D}$ 's (5.1) resides in the fact that the Hodge involution ${ }^{18}$

$$
*: \Lambda^{d} \longmapsto * \Lambda^{d}=\Lambda^{D-2-d}
$$

acts as a symmetry of the coset cohomology, where the differential forms of order $d$ are associated to $d$-fold antisymmetric irreps. $\Lambda^{d}$ of $\operatorname{SO}(D-2)=m c s(\operatorname{SL}(D-2, \mathbb{R}))$.

Note that, out of the possible forms belonging to the cohomology of $S O(D-2)=$ $m c s(\operatorname{SL}(D-2, \mathbb{R}))$, the coset $M_{N}^{D}(5.1)$ precisely singles out the physical massless $p>$ 0 forms of the corresponding supergravity theory with $\mathcal{N}=2 N$ supersymmetries in $D$ (Lorentzian) space-time dimensions. Indeed, by casting the Cartan decomposition of the cosets $M_{N}^{D}$ 's (5.1) in manifestly $\mathrm{SO}(D-2)$-covariant way, the Lie algebra of $M_{N}^{D}$ itself branches as

$$
\mathfrak{g}_{3}^{N} \ominus\left(\mathfrak{g}_{D}^{N} \oplus \mathfrak{s l}(D-2, \mathbb{R})\right) \sim \sum_{\substack{d \\ \text { manifestly } \operatorname{SO}(D-2) \text {-cov. }}} n_{d} \Lambda^{d}+\sum_{d} n_{d} * \Lambda^{d}
$$

where $\mathfrak{g}_{3}^{N}$ and $\mathfrak{g}_{D}^{N}$ respectively are the Lie algebras of $G_{3}^{N}$ and $G_{D}^{N}$, and $n_{d}$ is the (real) dimension of the relevant irreps. of the $U$-duality group $G_{D}^{N}$ in $D$ dimensions. Note that the r.h.s. of (5.103) is manifestly invariant under the Hodge involution $*$ (5.102). Thus, the vanishing character (5.2) of cosets $M_{N}^{D}$ 's (5.1) trivially follows from

$$
c\left(M_{N}^{D}\right)=\sum_{d} n_{d}\left(\begin{array}{c}
D-2 \\
d
\end{array}\right)=\sum_{d} n_{d}\left(\begin{array}{c}
D-2 \\
D-2-d
\end{array}\right)=n c\left(M_{N}^{D}\right) .
$$

By recalling formula (5.5), $c\left(M_{N}^{D}\right)=n c\left(M_{N}^{D}\right)$ can also be computed as the real dimension of the "mcs counterparts" of cosets $M_{N}^{D}$ 's (5.1), namely of the cosets $\widehat{M}_{N}^{D}(5.3)$.

In maximal theories $(N=16)$, by recalling the embedding $(2.2)$ and table 2 , one can trace back the fact that only $d$-fold antisymmetric irreps. $\Lambda^{d}$ 's occur in (5.103) to the embedding

$$
\begin{aligned}
\mathrm{SO}(16) & \supset \mathcal{R}_{D}^{16} \times \mathrm{SO}(D-2)_{J} \\
\mathbf{A d j}_{\mathrm{SO}(16)} & \equiv \mathbf{1 6} \times{ }_{a} \mathbf{1 6}=\mathbf{A d j}_{\mathrm{SO}(D-2)}+\sum_{d} n_{d} \Lambda^{d} .
\end{aligned}
$$

\footnotetext{
${ }^{18}$ The involutive or anti-involutive property $*^{2} \Lambda^{d}= \pm \Lambda^{d}$ generally depends on the signature and the dimension of the group manifold whose cohomology is under consideration. In this case, the relevant group is $\mathrm{SO}(D-2)=\operatorname{mcs}(S L(D-2, \mathbb{R}))$, and thus $*^{2} \Lambda^{d}=\Lambda^{d}$ for $D-2=4 n$, while $*^{2} \Lambda^{d}=-\Lambda^{d}$ for $D-2=4 n+2(n \in \mathbb{N})$.
} 
Namely, in $\mathrm{SO}(D-2)_{J}$ the antisymmetric rank-2 tensor product of spinor irreps. only contain antisymmetric $d$-fold irreps. (see e.g. [26]). We will consider here three examples, namely $D=11$ and $D=10$ (type IIA and IIB).

(I) In maximal supergravity $(N=16)$ in $D=11, d=3$ and $n_{d}=1$, thus (5.103) and (5.104) specifies as follows:

$$
\begin{aligned}
& \mathfrak{e}_{8(8)} \ominus \mathfrak{s l}(9, \mathbb{R}) \sim \Lambda^{3}+* \Lambda_{\text {manifestly SO(9)-cov. }}^{3}=\Lambda^{3}+\Lambda^{6}=\mathbf{8 4}+\mathbf{8 4} \\
& c\left(\frac{E_{8(8)}}{\mathrm{SL}(9, \mathbb{R})}\right)=\left(\begin{array}{l}
9 \\
3
\end{array}\right)=\left(\begin{array}{l}
9 \\
6
\end{array}\right)=n c\left(\frac{E_{8(8)}}{\mathrm{SL}(9, \mathbb{R})}\right)=84=\operatorname{dim}_{\mathbb{R}}\left(\frac{\mathrm{SO}(16)}{\mathrm{SO}(9)}\right) .
\end{aligned}
$$

In terms of the Cartan decomposition of the maximal non-symmetric Riemannian compact coset $\widehat{M}_{16}^{11}=\mathrm{SO}(16) / \mathrm{SO}(9)$, the result (5.107) can be obtained as a consequence of the maximal non-symmetric embedding ${ }^{19}$ (cfr. (2.2) and table 2)

$$
\begin{aligned}
\mathfrak{s o}(16) & \supset_{n s} \mathfrak{s o}(9) \\
\mathbf{1 6} & =\mathbf{1 6} \\
\mathbf{A d j}_{\mathrm{SO}(16)} & \equiv(\mathbf{1 6} \times \mathbf{1 6})_{a}=\underset{\mathbf{3 6}}{\mathbf{A d j}(9)}+\underset{\mathbf{8 4}}{\mathbf{A d j}_{\mathrm{SO}} .}
\end{aligned}
$$

(II) In maximal $D=10$ IIA supergravity, the relevant values are $d=1,2,3$ with $n_{1}=$ $n_{2}=n_{3}=1$, and thus (5.103) and (5.104) specifies as follows:

$$
\begin{aligned}
& \mathfrak{e}_{8(8)} \ominus(\mathfrak{s l}(8, \mathbb{R}) \oplus \mathfrak{s o}(1,1)) \\
& \sim \Lambda^{1}+\Lambda^{2}+\Lambda^{3}+* \Lambda^{1}+* \Lambda_{\text {manifestly }}^{2}=\Lambda^{3}(8) \text {-cov. } \\
&=\left(\mathbf{8}_{v}+\mathbf{2 8}+\mathbf{5 6}_{v}\right)+\left(\mathbf{8}_{v}+\mathbf{2 8}+\mathbf{5} \mathbf{6}_{v}\right) ; \\
& c\left(\frac{E_{8(8)}}{S O(1,1) \times S L(8, \mathbb{R})}\right)=8+\left(\begin{array}{l}
8 \\
2
\end{array}\right)+\left(\begin{array}{l}
8 \\
3
\end{array}\right)=\left(\begin{array}{l}
8 \\
7
\end{array}\right)+\left(\begin{array}{l}
8 \\
6
\end{array}\right)+\left(\begin{array}{l}
8 \\
5
\end{array}\right) \\
&=n c\left(\frac{E_{8(8)}}{S O(1,1) \times S L(8, \mathbb{R})}\right)=92=\operatorname{dim}_{\mathbb{R}}\left(\frac{S O(16)}{S O(8)}\right) .
\end{aligned}
$$

In terms of the Cartan decomposition of the non-maximal non-symmetric Riemannian compact coset $\widehat{M}_{16}^{10}$ IIA $=\mathrm{SO}(16) / \mathrm{SO}(8)$, the result $(5.110)$ can be obtained as a

\footnotetext{
${ }^{19}$ The embedding (5.108) actually follow from a Theorem due to Dynkin $[42,43]$, applied to the selfconjugate spinor irrep. 16 of $\mathrm{SO}(9)$ :

$$
\mathrm{SO}(9): \mathbf{1 6} \times{ }_{s} \mathbf{1 6}=\Lambda^{0}+\Lambda^{1}+\Lambda^{4}=\mathbf{1}+\mathbf{9}+\mathbf{1 2 6} .
$$
}


consequence of the next-to-maximal non-symmetric embedding $\left(\mathbf{A d j} \mathbf{j}=\Lambda^{2} ;(c f r\right.$.

and table 2)

$$
\begin{aligned}
& \mathfrak{s o}(16) \supset_{n s} \mathfrak{s o}(8) \\
& \mathbf{1 6}=\mathbf{8}_{s}+\mathbf{8}_{c} \\
& \mathbf{A d j}_{\mathrm{SO}(16)} \equiv(\mathbf{1 6} \times \mathbf{1 6})_{a}=\left(\mathbf{8}_{s}+\mathbf{8}_{c}\right) \times_{a}\left(\mathbf{8}_{s}+\mathbf{8}_{c}\right) \\
& 120 \\
& =\mathbf{8}_{s} \times{ }_{a} \mathbf{8}_{s}+\boldsymbol{8}_{c} \times{ }_{a} \boldsymbol{8}_{c}+\boldsymbol{8}_{s} \times \mathbf{8}_{c}=\underset{\mathbf{2 8}}{\mathbf{A d j}_{\mathrm{SO}(8)}}+\underset{\mathbf{8}_{v}}{\Lambda_{v}^{1}}+\underset{\mathbf{2 8}}{\Lambda^{2}}+\underset{\mathbf{5 6}_{v}}{\Lambda_{v}^{3}} .
\end{aligned}
$$

(III) In maximal $D=10$ IIB supergravity, the relevant values are $d=2,4$ with $n_{2}=$ $2 n_{4}=2$, and thus (5.103) and (5.104) specifies as follows:

$$
\begin{aligned}
\mathfrak{e}_{8(8)} \ominus(\mathfrak{s l}(8, \mathbb{R}) \oplus \mathfrak{s l}(2, \mathbb{R})) & \sim 2 \Lambda^{2}+\Lambda^{4}+2 * \begin{array}{l}
\Lambda^{1}+* \Lambda^{4}=2 \Lambda^{2}+\Lambda^{4}+2 \Lambda^{6}+\Lambda^{4} \\
\text { manifestly } \mathrm{SO}(8) \text {-cov. }
\end{array} \\
& =\left((\mathbf{2 8}, \mathbf{2})+\left(\mathbf{3 5}_{s}, \mathbf{1}\right)\right)+\left((\mathbf{2 8}, \mathbf{2})+\left(\mathbf{3 5}_{c}, \mathbf{1}\right)\right) ; \\
c\left(\frac{E_{8(8)}}{\mathrm{SO}(1,1) \times \mathrm{SL}(8, \mathbb{R})}\right) & =2\left(\begin{array}{l}
8 \\
2
\end{array}\right)+\left(\begin{array}{l}
8 \\
4
\end{array}\right)=2\left(\begin{array}{l}
8 \\
6
\end{array}\right)+\left(\begin{array}{l}
8 \\
4
\end{array}\right) \\
& =n c\left(\frac{E_{8(8)}}{\mathrm{SO}(1,1) \times \mathrm{SL}(8, \mathbb{R})}\right)=91 \\
& =\operatorname{dim}_{\mathbb{R}}\left(\frac{\mathrm{SO}(16)}{\mathrm{SO}(8) \times \mathrm{SO}(2)}\right) .
\end{aligned}
$$

In terms of the Cartan decomposition of the non-maximal non-symmetric Riemannian compact coset $\widehat{M}_{16}^{10 I I B}=\mathrm{SO}(16) /(\mathrm{SO}(8) \times \mathrm{SO}(2))$, the result $(5.113)$ can be obtained as a consequence of the next-to-maximal non-symmetric embedding ( $c f r$. (2.2) and table 2):

$$
\begin{aligned}
& \mathfrak{s o}(16) \supset_{n s} \mathfrak{s o}(8) \oplus \mathfrak{s o}(2) \\
& 16=\left(8_{s}, 2\right) \\
& \mathbf{A d j}_{\mathrm{SO}(16)} \equiv(\mathbf{1 6} \times \mathbf{1 6})_{a}=\left(\boldsymbol{8}_{s}, \mathbf{2}\right) \times_{a}\left(\boldsymbol{8}_{s}, \mathbf{2}\right)=\left(\mathbf{8}_{s} \times{ }_{a} \boldsymbol{8}_{s}, \mathbf{2} \times{ }_{s} \mathbf{2}\right)+\left(\boldsymbol{8}_{s} \times{ }_{s} \boldsymbol{8}_{s}, \mathbf{2} \times{ }_{a} \mathbf{2}\right) \\
& 120
\end{aligned}
$$

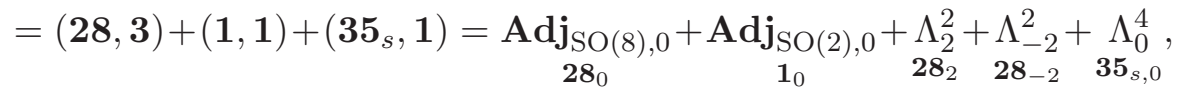

where in the last step the subscripts denote the charges of the $D=10$ IIB $\mathcal{R}$ symmetry $\mathfrak{s o}(2)$.

Finally, we present below the same analysis for other two "pure" supergravities:

( $I V)$ In $N=12$ supergravity (which shares the same bosonic sector of the quaternionic magical theory with $N=4[19,20])$ in $D=5, d=1$ and $n_{d}=15$, thus (5.103) 
and (5.104) specifies as follows:

$$
\begin{aligned}
\mathfrak{e}_{7(-5)} \ominus\left(\mathfrak{s u}^{*}(6) \oplus \mathfrak{s l}(3, \mathbb{R})\right) & \sim(14+1) \Lambda^{1}+(14+1) * \Lambda^{1}=(14+1) \Lambda^{1}+(14+1) \Lambda^{2} \\
& =(14+1) \mathbf{3}+(14+1) \mathbf{3} \\
c\left(\frac{E_{7(-5)}}{S U^{*}(6) \times \mathrm{SL}(3, \mathbb{R})}\right) & =(14+1) 3=(14+1)\left(\begin{array}{l}
3 \\
2
\end{array}\right)=n c\left(\frac{E_{7(-5)}}{S U^{*}(6) \times \mathrm{SL}(3, \mathbb{R})}\right) \\
& =45=\operatorname{dim}_{\mathbb{R}}\left(\frac{\mathrm{SO}(12)}{\mathrm{USp}(6)}\right) .
\end{aligned}
$$

In terms of the Cartan decomposition of the maximal non-symmetric Riemannian compact coset $\widehat{M}_{12}^{5}=\mathrm{SO}(12) / \mathrm{USp}(6)$, the result (5.116) can be obtained as a consequence of the maximal non-symmetric embedding

$$
\begin{aligned}
\mathfrak{s o}(12) & \supset_{n s} \mathbf{u s p}(6) \oplus \mathfrak{s u}(2) \\
\mathbf{1 2} & =(\mathbf{6}, \mathbf{2}) \\
\mathbf{A d j}_{\mathbf{6 O}(12)} & \equiv(\mathbf{1 2} \times \mathbf{1 2})_{a}=(\mathbf{6}, \mathbf{2}) \times_{a}(\mathbf{6}, \mathbf{2})=\left(\mathbf{6} \times{ }_{a} \mathbf{6}, \mathbf{2} \times{ }_{s} \mathbf{2}\right)+\left(\mathbf{6} \times{ }_{s} \mathbf{6}, \mathbf{2} \times{ }_{a} \mathbf{2}\right) \\
& =(\mathbf{1 4}, \mathbf{3})+\underset{\underset{\mathbf{A d j}}{(\mathbf{1}, \mathbf{3})}+(\mathbf{2 1}, \mathbf{1})}{\left(\operatorname{Adj}_{\mathrm{USp}(6)}\right)}
\end{aligned}
$$

where the $D=5$ massless spin algebra $\mathfrak{s u}(2)$ is not modded out in order to determine $\widehat{M}_{12}^{5}$, and it corresponds to the "extra" $\operatorname{USp}(6)(\mathcal{R}$-symmetry-)singlet, a peculiar feature of this extended supergravity theory (which makes it amenable to an $N=4$ interpretation).

$(V)$ In $N=10$ supergravity in $D=4, d=1$ and $n_{d}=10$, thus (5.103) and (5.104) specifies as follows:

$$
\begin{aligned}
\mathfrak{e}_{6(-14)} \ominus(\mathfrak{s u}(5,1) \oplus \mathfrak{s l}(2, \mathbb{R})) & \sim 10 \Lambda^{1}+\underset{\text { manifestly } \mathrm{SO}(2) \text {-cov. }}{10 * \Lambda^{1}=10 \Lambda^{1}+10 \Lambda^{1}=(10) \mathbf{2}+(10) \mathbf{2}} \\
c\left(\frac{E_{6(-14)}}{\mathrm{SU}(5,1) \times \mathrm{SL}(2, \mathbb{R})}\right) & =10 \cdot 2=n c\left(\frac{E_{6(-14)}}{\operatorname{SU}(5,1) \times \mathrm{SL}(2, \mathbb{R})}\right) \\
& =40=\operatorname{dim}_{\mathbb{R}}\left(\frac{\mathrm{SO}(10)}{\mathrm{U}(5)}\right) .
\end{aligned}
$$

In terms of the Cartan decomposition of the maximal non-symmetric Riemannian compact coset $\widehat{M}_{10}^{4}=\mathrm{SO}(10) / \mathrm{U}(5)$, the result (5.118) can be obtained as a consequence of the maximal symmetric embedding

$$
\begin{aligned}
\mathfrak{s o}(10) & \supset_{s} \mathfrak{s u}(5) \oplus \mathfrak{u}(1) \\
\mathbf{1 0} & =\mathbf{5}_{1}+\overline{\mathbf{5}}_{-1} \\
\operatorname{Adj}_{\mathrm{SO}(10)} & \equiv(\mathbf{1 0} \times \mathbf{1 0})_{a}=\left(\mathbf{5}_{1}+\overline{\mathbf{5}}_{-1}\right) \times_{a}\left(\mathbf{5}_{1}+\overline{\mathbf{5}}_{-1}\right) \\
& =\mathbf{5}_{1} \times_{a} \mathbf{5}_{1}+\overline{\mathbf{5}}_{-1} \times_{a} \overline{\mathbf{5}}_{-1}+\mathbf{5}_{1} \times \overline{\mathbf{5}}_{-1}=\mathbf{1 0}_{2}+\overline{\mathbf{1 0}}_{-2}+\mathbf{2 4}_{0}+\mathbf{1}_{0}
\end{aligned}
$$

where the subscripts denote the charges with respect to the $D=4$ massless spin algebra $\mathfrak{u}(1)$. 


\section{Conclusion}

In this paper we have analyzed some consequences of the super-Ehlers structure of $N$ extended supergravity theories in $D \geqslant 4$ space-time dimensions. As the Ehlers $\operatorname{SL}(D-2, \mathbb{R})$ is an off-shell symmetry of the Lagrangian [10-12], so there should exist an Hamiltonian formulation of light-cone supergravity in which $U$-duality $G_{N}^{D}$ is an off-shell symmetry. Moreover, at least for any amount of supersymmetry $N \geqslant 4$, the Ehlers group can be regarded as the commutant of $G_{N}^{D}$ itself inside the $U$-duality group $G_{N}^{3}$ in $D=3$.

The pseudo-Riemannian manifolds pertaining to the embedding of the super-Ehlers group $G_{N}^{D} \times \operatorname{SL}(D-2, \mathbb{R})$ into $G_{N}^{3}$, namely the cosets $M_{N}^{D}$ 's $(5.1)$, have been found to exhibit an interesting invariance under the Hodge involution (5.102), acting on the cohomology of $M_{N}^{D}$, which in turn singles out only the physical massless $p>0$ forms of the corresponding supergravity theory, regarded as $p$-fold antisymmetric irreps. $\Lambda^{p}$ of the massless spin group $\mathrm{SO}(D-2)_{J}=m c s\left(\mathrm{SL}(D-2, \mathbb{R})_{\text {Ehlers }}\right)$ in $D$ (Lorentzian) space-time dimensions.

The symmetry under the Hodge involution (5.102) implies all the cosets $M_{N}^{D}$ 's (5.1) to have a vanishing character, namely to have the same number of compact and non-compact generators: $c\left(M_{N}^{D}\right)=n c\left(M_{N}^{D}\right)$. Such a number, along with its manifestly $\operatorname{SO}(D-2)_{J^{-}}$ covariant decomposition in terms of physical massless $p>0$ forms, can be computed by considering the Cartan decomposition of the cosets $\widehat{M}_{N}^{D}$ 's $(5.3)$, which can be regarded as the " $m c s$ counterpart" of $M_{N}^{D}$ 's (5.1). Indeed, the embedding of $\operatorname{SO}(D-2)_{J}$ inside $H_{N}^{3} \equiv m c s\left(G_{N}^{3}\right)$ is such that the generators of $\widehat{M}_{N}^{D}$ split only into antisymmetric tensor irreps. of $\mathrm{SO}(D-2)_{J}$ itself, with multiplicities given by irreps. of $H_{N}^{D} \equiv m c s\left(G_{N}^{D}\right)$.

The approach of this paper may be relevant for the analysis of the issue of ultraviolet divergences in supergravity theories with maximal or non-maximal supersymmetry, by exploiting the light-cone formulation, along the lines e.g. of [1, 10-12].

\section{Acknowledgments}

We would like to thank V. S. Varadarajan for an enlightening discussion on the Hodge involution.

A.M. would like to thank the Department of Physics and Astronomy, University of California at Los Angeles, where this project was completed, for kind hospitality and stimulating environment.

The work of S.F. has been supported by the ERC Advanced Grant no. 226455, Supersymmetry, Quantum Gravity and Gauge Fields (SUPERFIELDS), and in part by DOE Grant DE-FG03-91ER40662.

\section{A Embeddings}

Let us start by recalling some useful definitions.

Given two semisimple Lie groups $G^{\prime}$ and $G$, generated by the Lie algebras $\mathfrak{g}^{\prime}, \mathfrak{g}$, respectively, if $G^{\prime} \subset G$ (proper inclusion), we say that $G^{\prime}$ is maximal in $G$ iff there is no proper subalgebra $\mathfrak{g}^{\prime \prime}$ of $\mathfrak{g}$ containing $\mathfrak{g}^{\prime}$. If $G^{\prime}$ and $G$ are complex semisimple Lie groups such that 


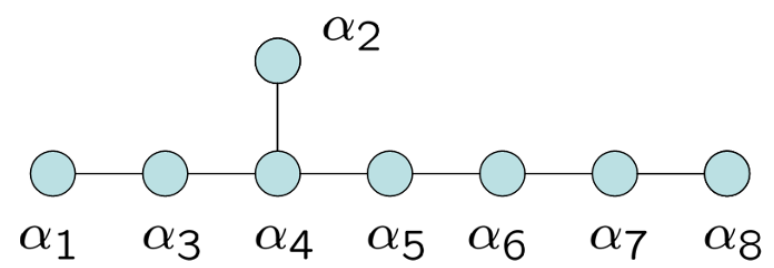

Figure 1. $\mathfrak{e}_{8(8)}$ Dynkin diagram.

$G^{\prime} \subset G$, the embedding of $G^{\prime}$ into $G$ is regular iff one can find a basis of $\mathfrak{g}^{\prime}$ consisting of elements of a Cartan subalgebra $\mathfrak{h}$ of $\mathfrak{g}$ and shift-generators $E_{\alpha}$ corresponding to roots $\alpha$ of $\mathfrak{g}$ relative to $\mathfrak{h}[42]$. Regular subalgebras $\mathfrak{g}^{\prime}$ of a semisimple Lie algebra $\mathfrak{g}$ can be constructed using the simple procedure defined by Dynkin in [42]: the Dynkin diagram of $\mathfrak{g}^{\prime}$ can be obtained as a truncation of the extended diagram of $\mathfrak{g}$. When considering real forms $G^{\prime}, G$ of complex semisimple Lie groups $G_{\mathbb{C}}^{\prime}, G_{\mathbb{C}}$, we say that $G^{\prime} \subset G$ is regularly embedded in $G$ iff the complexification $\mathfrak{g}_{\mathbb{C}}^{\prime}$ of $\mathfrak{g}^{\prime}$ is regularly embedded in the complexification $\mathfrak{g}_{\mathbb{C}}$ of $\mathfrak{g}$. The embedding of $G^{\prime}$ into $G$ is symmetric iff we can write $\mathfrak{g}=\mathfrak{g}^{\prime} \oplus \mathfrak{p}$, such that $\left[\mathfrak{g}^{\prime}, \mathfrak{p}\right] \subset \mathfrak{p}$ and $[\mathfrak{p}, \mathfrak{p}] \subset \mathfrak{g}^{\prime}$. Finally the embedding is rank-preserving iff $\operatorname{rank}\left(\mathfrak{g}^{\prime}\right)=\operatorname{rank}(\mathfrak{g})$.

\section{A.1 The embeddings $\operatorname{SL}(D-2, \mathbb{R}) \times \mathrm{E}_{11-D(11-D)} \subset \mathrm{E}_{8(8)}$}

The $\boldsymbol{D}=\mathbf{5}$ case $\mathbf{S L}(\mathbf{3}, \mathbb{R}) \times \mathbf{E}_{\mathbf{6}(\mathbf{6})} \subset \mathbf{E}_{\mathbf{8}(\mathbf{8})} . \quad$ The embedding of $\mathfrak{s l}(3, \mathbb{R}) \oplus \mathfrak{e}_{6(6)} \subset \mathfrak{e}_{8(8)}$ is regular and can be described using Dynkin's construction [42]. Let us number the simple roots of $\mathfrak{e}_{8(8)}$ so that the $D_{7}$ sub-Dynkin diagram consists of the roots $\alpha_{2}, \ldots, \alpha_{8}$, with $\alpha_{2}$ and $\alpha_{3}$ on the two symmetric legs, and $\alpha_{1}$ is the $D_{7}$-spinor weight attached to $\alpha_{3}$, see figure 1. The $\mathfrak{e}_{8(8)}$ Cartan matrix reads:

$$
\left\langle\alpha_{i}, \alpha_{j}\right\rangle=\left(\begin{array}{cccccccc}
2 & 0 & -1 & 0 & 0 & 0 & 0 & 0 \\
0 & 2 & 0 & -1 & 0 & 0 & 0 & 0 \\
-1 & 0 & 2 & -1 & 0 & 0 & 0 & 0 \\
0 & -1 & -1 & 2 & -1 & 0 & 0 & 0 \\
0 & 0 & 0 & -1 & 2 & -1 & 0 & 0 \\
0 & 0 & 0 & 0 & -1 & 2 & -1 & 0 \\
0 & 0 & 0 & 0 & 0 & -1 & 2 & -1 \\
0 & 0 & 0 & 0 & 0 & 0 & -1 & 2
\end{array}\right)
$$

In an orthonormal basis the simple roots $\alpha_{i}$ read:

$$
\begin{aligned}
& \alpha_{1}=-\frac{1}{2}\left(\epsilon_{1}+\epsilon_{2}+\epsilon_{3}+\epsilon_{4}+\epsilon_{5}+\epsilon_{6}-\epsilon_{7}-\epsilon_{8}\right), \\
& \alpha_{2}=\epsilon_{6}+\epsilon_{7} ; \alpha_{3}=\epsilon_{6}-\epsilon_{7} ; \alpha_{4}=\epsilon_{5}-\epsilon_{6} ; \alpha_{5}=\epsilon_{4}-\epsilon_{5} ; \alpha_{6}=\epsilon_{3}-\epsilon_{4} ; \alpha_{7}=\epsilon_{2}-\epsilon_{3} ; \\
& \alpha_{8}=\epsilon_{1}-\epsilon_{2}
\end{aligned}
$$

Let us denote by $\Delta_{+}\left[\mathfrak{e}_{8(8)}\right]=\left\{\alpha=\sum_{i=1}^{8} n^{i} \alpha_{i}\right\}$ the set of positive roots of $\mathfrak{e}_{8(8)}$. The $\mathfrak{e}_{6(6)}$ subalgebra is defined by the sub-Dynkin diagram consisting of the simple roots $\alpha_{a}$, 
$a=1, \ldots, 6$. The 36 positive $\mathfrak{e}_{6(6)}$-roots be denoted by $\gamma_{A}$, so that:

$$
\Delta_{+}\left[\mathfrak{e}_{6(6)}\right]=\left\{\gamma_{A}=\sum_{a=1}^{6} n_{A}^{a} \alpha_{a}\right\}
$$

Furthermore let us consider the following positive roots $\beta_{x}, x=1,2,3$ :

$$
\begin{aligned}
& \beta_{1}=\alpha_{8}=\epsilon_{1}-\epsilon_{2} ; \quad \beta_{2}=2 \alpha_{1}+3 \alpha_{2}+4 \alpha_{3}+6 \alpha_{4}+5 \alpha_{5}+4 \alpha_{6}+3 \alpha_{7}+\alpha_{8}=\epsilon_{2}+\epsilon_{8} \\
& \beta_{3}=2 \alpha_{1}+3 \alpha_{2}+4 \alpha_{3}+6 \alpha_{4}+5 \alpha_{5}+4 \alpha_{6}+3 \alpha_{7}+2 \alpha_{8}=\epsilon_{1}+\epsilon_{8} .
\end{aligned}
$$

One can easily verify that $\beta_{x}$ generate an $\mathfrak{s l}(3, \mathbb{R})$-root space which is orthogonal to $\Delta_{+}\left[\mathfrak{e}_{6(6)}\right]: \beta_{x} \cdot \gamma_{A}=0$. We have then constructed an $\mathfrak{s l}(3, \mathbb{R}) \oplus \mathfrak{e}_{6(6)}$ subalgebra of $\mathfrak{e}_{8(8)}$ :

$$
\begin{aligned}
\mathfrak{s l}(3, \mathbb{R}) & =\operatorname{Span}\left(H_{\beta_{1}}, H_{\beta_{2}}, E_{ \pm \beta_{1}}, E_{ \pm \beta_{2}}, E_{ \pm \beta_{3}}\right), \\
\mathfrak{e}_{6(6)} & =\operatorname{Span}\left(H_{\alpha_{a}}, E_{ \pm \gamma_{A}}\right)_{\substack{a=1, \ldots, 6 \\
A=1, \ldots, 36}}
\end{aligned}
$$

Within $\mathfrak{s l}(3, \mathbb{R}) \oplus \mathfrak{e}_{6(6)}$ we can identify its maximal compact subalgebra $\mathfrak{s o}(3) \oplus \mathfrak{u} \mathfrak{s p}(8)$, which is a maximal subalgebra of $\mathfrak{s o}(16)$ :

$$
\begin{aligned}
\mathfrak{s o}(16) & =\operatorname{Span}\left(E_{\alpha}-E_{-\alpha}\right)_{\alpha \in \Delta_{+}\left[\mathfrak{e}_{8(8)}\right]}, \\
\mathfrak{s o}(3) & =\operatorname{Span}\left(E_{\beta_{x}}-E_{-\beta_{x}}\right)_{x=1,2,3}, \\
\mathfrak{u s p}(8) & =\operatorname{Span}\left(E_{\gamma_{A}}-E_{-\gamma_{A}}\right)_{A=1, \ldots, 36} .
\end{aligned}
$$

With respect to this $\mathrm{SO}(3) \times \mathrm{USp}(8)$ subgroup of $\mathrm{SO}(16)$ the coset space

$$
\mathfrak{K}=\mathfrak{e}_{8(8)} \ominus \mathfrak{s o}(16)=\operatorname{Span}\left(H_{\alpha_{i}}, E_{\alpha}+E_{-\alpha}\right)_{\alpha \in \Delta_{+}\left[\mathfrak{e}_{8(8)}\right] ; i=1, \ldots, 8},
$$

should decompose as follows:

$$
\begin{aligned}
\mathfrak{K} & =\mathfrak{K}[\mathfrak{s l}(3, \mathbb{R})] \oplus \mathfrak{K}\left[\mathfrak{e}_{6}(6)\right] \oplus(\mathbf{3}, \mathbf{2 7}), \\
\mathfrak{K}[\mathfrak{s l}(3, \mathbb{R})] & =\mathfrak{s l}(3, \mathbb{R}) \ominus \mathfrak{s o}(3)=\operatorname{Span}\left(H_{\beta_{1}}, H_{\beta_{2}}, E_{\beta_{x}}+E_{-\beta_{x}}\right)_{x=1,2,3}=(\mathbf{5}, \mathbf{1}), \\
\mathfrak{K}\left[\mathfrak{e}_{6(6)}\right] & =\mathfrak{e}_{6(6)} \ominus \mathfrak{u} \mathfrak{s p}(8)=\operatorname{Span}\left(H_{\alpha_{a}}, E_{\gamma_{A}}+E_{-\gamma_{A}}\right)_{\substack{a=1, \ldots, 6 \\
A=1, \ldots, 36}}=(\mathbf{1}, \mathbf{4 2}),
\end{aligned}
$$

Generalizing to $\operatorname{SL}(D-\mathbf{2}, \mathbb{R}) \times \mathbf{E}_{11-D(11-D)} \subset \mathbf{E}_{\mathbf{8}(8)}$. The above construction is extended to define the embedding of $\mathfrak{s l}(D-2, \mathbb{R}) \oplus \mathfrak{e}_{11-D(11-D)} \subset \mathfrak{e}_{8(8)}, D \geq 4$, following the same recipe by Dynkin. The embedding of $\mathfrak{e}_{11-D(11-D)}$ is defined by deleting in the $\mathfrak{e}_{8(8)}$ Dynkin diagram the last $D-3$ simple roots to the right, namely $\alpha_{12-D}, \ldots, \alpha_{8}$, see figure 2. The set of positive roots of $\mathfrak{e}_{11-D(11-D)}$ reads:

$$
\Delta_{+}\left[\mathfrak{e}_{11-D(11-D)}\right]=\left\{\gamma_{A}\right\}=\left\{\epsilon_{a} \pm \epsilon_{b},\left[\frac{\epsilon_{8}}{2}-\sum_{\alpha=1}^{D-3} \frac{\epsilon_{\alpha}}{2}+\left(\sum_{a=D-2}^{7} \pm \frac{\epsilon_{a}}{2}\right)_{\text {odd }+}\right]\right\},
$$

where those in square brackets are the weights of a chiral spinorial representation of the $\mathfrak{s o}(10-D, 10-D)$ subalgebra of $\mathfrak{e}_{11-D(11-D)}$. The set of positive roots of $\mathfrak{s l}(D-2, \mathbb{R})$ reads:

$$
\Delta_{+}[\mathfrak{s l}(D-2, \mathbb{R})]=\left\{\beta_{x}\right\}=\left\{\epsilon_{\alpha}-\epsilon_{\beta}, \epsilon_{\alpha}+\epsilon_{8}\right\}
$$




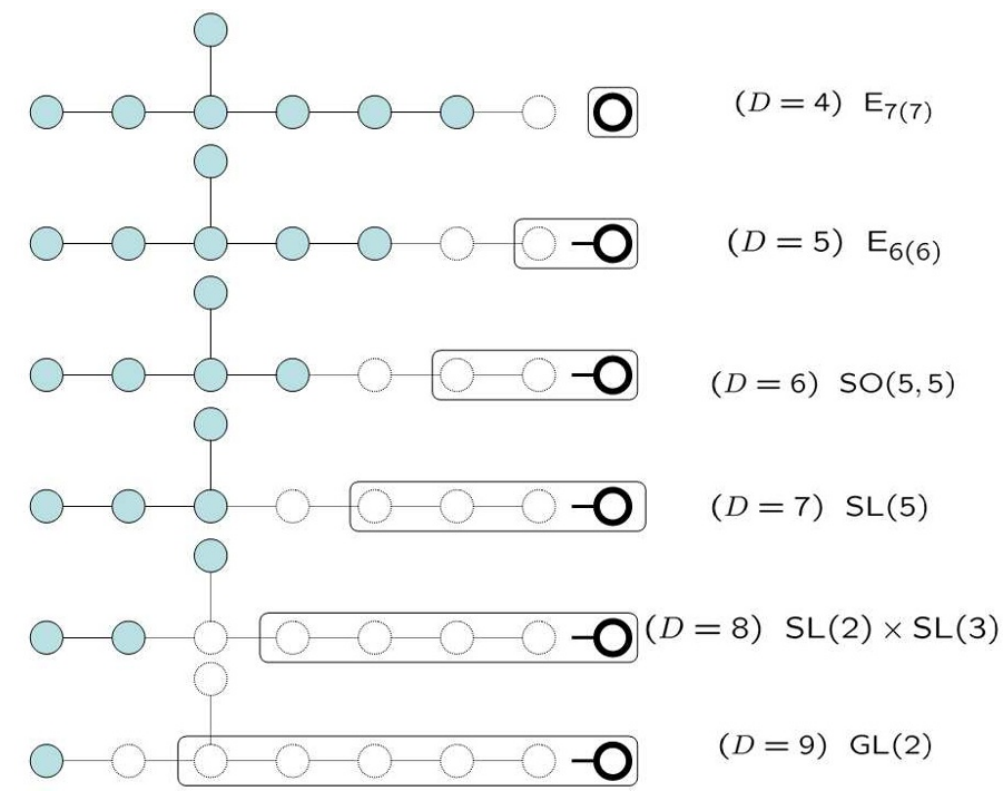

Figure 2. The filled circles define the $\mathfrak{e}_{11-D(11-D)}$ sub-Dynkin diagram, while the thick circle represents the exceptional root $-\psi, \psi=\epsilon_{1}+\epsilon_{8}$ being the highest root of $\mathfrak{e}_{8}$, which, together with the other roots in the rectangles, defines the Dynkin diagram of $\mathfrak{s l}(D-2, \mathbb{R})$.

where $\alpha, \beta=1, \ldots, D-3, \beta>\alpha$ and $x=1, \ldots,(D-3)(D-2) / 2$. One can easily verify that the two root systems are orthogonal, namely: $\beta_{x} \cdot \gamma_{A}=0$.

This defines the $\mathfrak{s l}(D-2, \mathbb{R}) \oplus \mathfrak{e}_{11-D(11-D)}$ subalgebra of $\mathfrak{e}_{8(8)}$ :

$$
\begin{aligned}
\mathfrak{s l}(D-2, \mathbb{R}) & =\operatorname{Span}\left(H_{\alpha_{13-D}}, \ldots, H_{\alpha_{8}}, H_{\epsilon_{1}+\epsilon_{8}}, E_{ \pm \beta_{x}}\right)_{\beta_{x} \in \Delta_{+}[\mathfrak{s l}(D-2, \mathbb{R})]} \\
\mathfrak{e}_{11-D(11-D)} & =\operatorname{Span}\left(H_{\alpha_{a}}, E_{ \pm \gamma_{A}}\right)_{\substack{a=1, \ldots, 11-D \\
\gamma_{A} \in \Delta_{+}\left[\mathfrak{e}_{11-D(11-D)}\right]}}, D=4, \ldots, 8 \\
\mathfrak{e}_{2(2)} & =\operatorname{Span}\left(H_{\alpha_{1}}, H_{\lambda}, E_{ \pm \alpha_{1}}\right), D=9
\end{aligned}
$$

where the generators $H_{\alpha_{13-D}}, \ldots, H_{\alpha_{8}}$, in the first line, are not counted for $D=4$, for which the only Cartan generator of $\mathfrak{s l}(2, \mathbb{R})$ is $H_{\epsilon_{1}+\epsilon_{8}}$. In the $D=9$ case, the vector $\lambda$ in the last line is: $\lambda=\epsilon_{7}-\alpha_{1} / 4$ and is orthogonal to the $\beta_{x}$ and to $\alpha_{1}$.

As far as the corresponding maximal compact subalgebra $\mathfrak{s o}(D-2) \oplus \mathfrak{H}_{D}$ is concerned, its can be constructed as follows:

$$
\begin{aligned}
\mathfrak{s o}(D-2) & =\operatorname{Span}\left(E_{\beta_{x}}-E_{-\beta_{x}}\right)_{\beta_{x} \in \Delta_{+}[\mathfrak{s l}(D-2, \mathbb{R})]}, \\
\mathfrak{H}_{D} & =\operatorname{Span}\left(E_{\gamma_{A}}-E_{-\gamma_{A}}\right)_{\gamma_{A} \in \Delta_{+}\left[\mathfrak{e}_{11-D(11-D)}\right]}
\end{aligned}
$$

where $\mathfrak{H}_{D}$ is the maximal compact subalgebra of $\mathfrak{e}_{11-D(11-D)}$.

In the $D=10$ case we need to consider the type IIA and type IIB descriptions in which the relevant subgroups of $E_{8(8)}$ are $\mathrm{SL}(8, \mathbb{R}) \times \mathrm{SO}(1,1)$ and $\mathrm{SL}(8, \mathbb{R})^{\prime} \times \mathrm{SL}(2, \mathbb{R})$, respectively. ${ }^{20}$ Their embeddings are illustrated in figure 6 . In the former case the $U$ duality group is $\mathrm{SO}(1,1)$ and is generated by the Cartan generator $\sum_{i=1}^{8} H_{\epsilon_{i}}-2 H_{\epsilon_{8}}$.

\footnotetext{
${ }^{20}$ We shall omit the prime in the following.
} 


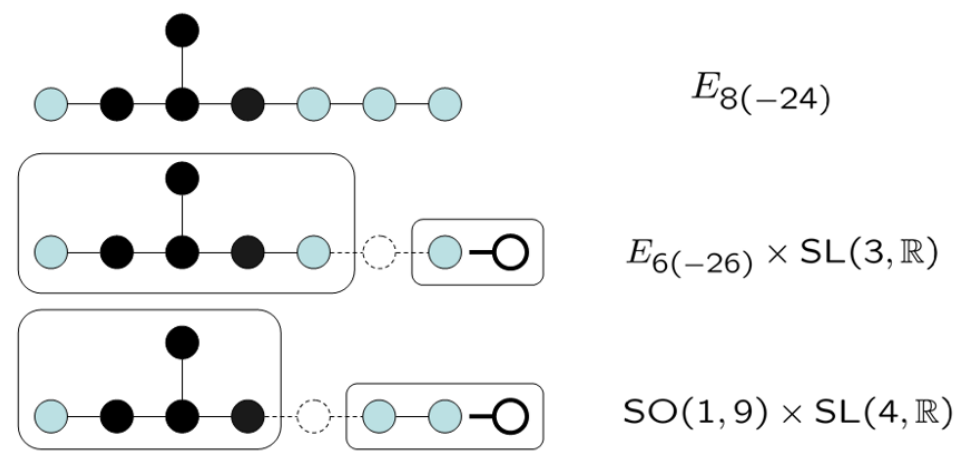

Figure 3. From top to bottom: Satake diagram of $\mathfrak{e}_{8(-24)}$ and embeddings of the $\mathfrak{e}_{6(-26)} \oplus \mathfrak{s l}(3, \mathbb{R})$ and $\mathfrak{s o}(1,9) \oplus \mathfrak{s l}(4, \mathbb{R})$ diagrams inside the extension of the $\mathfrak{e}_{8(-24)}$ one.

\section{A.2 Other embeddings}

Embeddings considered here were also dealt with in [17]. Here we provide a detailed and explicit construction of a number of embeddings in terms of the generators of the corresponding Lie algebras, using the notation of [14]. Let us start discussing in detail the embeddings of $E_{6(-26)} \times \mathrm{SL}(3, \mathbb{R})$ and $\mathrm{SO}(1,9) \times \mathrm{SL}(4, \mathbb{R})$ inside $E_{8(-24)}$. At the level of the corresponding Lie algebras, these embeddings are illustrated in figure 3 , where the Satake diagrams of $\mathfrak{e}_{6(-26)} \oplus \mathfrak{s l}(3, \mathbb{R})$ and $\mathfrak{s o}(1,9) \oplus \mathfrak{s l}(4, \mathbb{R})$ are obtained from the $\mathfrak{e}_{8(-24)}$ one once again using Dynkin's procedure of extending the latter and canceling a suitable simple root. Let us briefly review the definition of Satake diagrams for non-split (i.e. nonmaximally-non-compact) Lie algebras and the construction of the $\mathfrak{e}_{6(-26)} \oplus \mathfrak{s l}(3, \mathbb{R})$ and $\mathfrak{s o}(1,9) \oplus \mathfrak{s l}(4, \mathbb{R})$ generators in terms of a canonical basis of the complex $\mathfrak{e}_{8}$. The latter consists of a basis $\left\{H_{\epsilon_{i}}\right\}, i=1 \ldots, 8$, of Cartan generators, with respect to which the $\mathfrak{e}_{8}$ roots are defined, and shift operators $E_{\alpha}, E_{-\alpha}, \alpha$ being the 120 positive roots. The real form $\mathfrak{e}_{8(-24)}$ is characterized by a Cartan subalgebra $\mathfrak{h}$ which splits into the direct sum of a subspace $\mathfrak{h}^{n c}$ of non-compact generators (i.e. generators which are odd with respect to the Cartan involution $\tau^{21}$ ) and a subspace $\mathfrak{h}^{c}$ of compact generators, defined in terms of the $\left\{H_{\epsilon_{i}}\right\}$ as follows:

$$
\mathfrak{h}=\mathfrak{h}^{n c} \oplus \mathfrak{h}^{c} ; \mathfrak{h}^{c}=\operatorname{Span}\left(i H_{\alpha_{2}}, i H_{\alpha_{3}}, i H_{\alpha_{4}}, i H_{\alpha_{5}}\right) ; \mathfrak{h}^{n c}=\operatorname{Span}\left(H_{\epsilon_{1}}, H_{\epsilon_{2}}, H_{\epsilon_{3}}, H_{\epsilon_{8}}\right)
$$

Note that $\mathfrak{h}^{c}$ is the Cartan subalgebra of an $\mathfrak{s o}(8)$ subalgebra of $\mathfrak{e}_{8(-24)}$ whose Dynkin diagram is defined by the black roots in figure 3 . The $\mathfrak{e}_{8}$ positive roots split into a $12-$ dimensional sub-space $\Delta_{+}^{(0)}\left[\mathfrak{e}_{8}\right]$ of roots having null restriction to $\mathfrak{h}^{n c}$ and a 108-dimensional

\footnotetext{
${ }^{21}$ We can always find a suitable basis for the matrix representation of the generators so that $\tau(M)=-M^{\dagger}$. This means that we shall regard compactness and non-compactness of a generator to be synonyms, in any matrix representation, of being anti-hermitian and hermitian, respectively. Moreover, in our conventions, $E_{-\alpha}=-\tau\left(E_{\alpha}\right)=E_{\alpha}^{\dagger}$.
} 
space $\bar{\Delta}_{+}\left[\mathfrak{e}_{8}\right]$ of roots with a non-trivial restriction to $\mathfrak{h}^{n c}$ :

$$
\Delta_{+}\left[\mathfrak{e}_{8}\right]=\Delta_{+}^{(0)}\left[\mathfrak{e}_{8}\right] \oplus \bar{\Delta}_{+}\left[\mathfrak{e}_{8}\right] .
$$

The conjugation $\sigma$ with respect to $\mathfrak{e}_{8(-24)}$ is the conjugation on the complex $\mathfrak{e}_{8}$ which leaves the elements of the subalgebra $\mathfrak{e}_{8(-24)}$ invariant. It defines a correspondence between $\mathfrak{e}_{8-}$ roots $\alpha \leftrightarrow \alpha^{\sigma}$ such that $\sigma\left(E_{\alpha}\right) \propto E_{\alpha^{\sigma}}$. The couple of roots $\alpha, \alpha^{\sigma}$ satisfies the property:

$$
\alpha_{\mid \mathfrak{h}^{n c}}=\alpha_{\mid \mathfrak{h}^{n c}}^{\sigma} ; \quad \alpha_{\mid \mathfrak{h}^{c}}=-\alpha_{\mid \mathfrak{h}^{c}}^{\sigma} .
$$

Clearly if $\alpha \in \Delta_{+}^{(0)}\left[\mathfrak{e}_{8}\right], \alpha^{\sigma}=-\alpha$, while if $\alpha \in \bar{\Delta}_{+}\left[\mathfrak{e}_{8}\right]$ and $\alpha_{\mid \mathfrak{h}^{c}}=0$, we have $\alpha^{\sigma}=\alpha$. Thus if $\alpha \in \bar{\Delta}_{+}\left[\mathfrak{e}_{8}\right]$, to each couple of nilpotent generators $E_{\alpha}$ and $\sigma\left(E_{\alpha}\right)$ in $\mathfrak{e}_{8}$, there corresponds a couple of nilpotent generators in $\mathfrak{e}_{8(-24)}$ given by the $\sigma$-invariant combinations $i\left(E_{\alpha}-\right.$ $\left.\sigma\left(E_{\alpha}\right)\right), E_{\alpha}+\sigma\left(E_{\alpha}\right)$, which can be both brought to an upper-triangular form, for all $\alpha$. If, on the other hand, $\alpha \in \Delta_{+}^{(0)}\left[\mathfrak{e}_{8}\right]$, the same combinations define compact $\mathfrak{s o}(8)$ generators $i\left(E_{\alpha}+E_{-\alpha}\right), E_{\alpha}-E_{-\alpha}$.

To summarize, the $\mathfrak{e}_{8(-24)}$ generators can be expressed in terms of the $\mathfrak{e}_{8}$ canonical basis as follows:

$$
\begin{aligned}
\mathfrak{e}_{8(-24)} & =\mathfrak{h} \oplus \mathfrak{l}_{+} \oplus \mathfrak{l}_{-} \oplus \mathfrak{m}_{0}, \\
\mathfrak{l}_{+} & =\operatorname{Span}\left[i\left(E_{\alpha}-\sigma\left(E_{\alpha}\right)\right), E_{\alpha}+\sigma\left(E_{\alpha}\right)\right]_{\left(\alpha, \alpha^{\sigma}\right) \in \bar{\Delta}_{+}\left[\mathfrak{e}_{8}\right]}, \\
\mathfrak{l}_{-} & =\operatorname{Span}\left[i\left(E_{-\alpha}-\sigma\left(E_{-\alpha}\right)\right), E_{-\alpha}+\sigma\left(E_{-\alpha}\right)\right]_{\left(\alpha, \alpha^{\sigma}\right) \in \bar{\Delta}_{+}\left[\mathfrak{e}_{8}\right]}, \\
\mathfrak{m}_{0} & =\operatorname{Span}\left[i\left(E_{\alpha}+E_{-\alpha}\right), E_{\alpha}-E_{-\alpha}\right]_{\alpha \in \Delta_{+}^{(0)}\left[\mathfrak{e}_{8}\right]},
\end{aligned}
$$

The 112-dimensional solvable Lie algebra $\mathfrak{s}_{0}=\mathfrak{h}^{n c} \oplus \mathfrak{l}_{+}$is the one defined by the Iwasawa decomposition of $\mathfrak{e}_{8(-24)}$ with respect to $\mathfrak{e}_{7(-133)} \oplus \mathfrak{s u}(2)$, and its generators, in a suitable basis, can all be represented by upper-triangular matrices. The centralizer of $\mathfrak{h}^{n c}$ is the $\mathfrak{s o}(8)$ subalgebra given by $\mathfrak{h}^{c} \oplus \mathfrak{m}_{0}$ and is also contained inside the subalgebras $\mathfrak{e}_{6(-26)}$ and $\mathfrak{s o}(1,9)$, as it is apparent from figure 3 .

The $\mathfrak{e}_{6(-26)}$ generators in terms of the above $\mathfrak{e}_{8(-24)}$ ones are easily written:

$$
\begin{aligned}
\mathfrak{e}_{6(-26)} & =\mathfrak{h}^{\prime} \oplus \mathfrak{l}_{+}^{\prime} \oplus \mathfrak{l}_{-}^{\prime} \oplus \mathfrak{m}_{0}, \\
\mathfrak{l}_{+}^{\prime} & =\operatorname{Span}\left[i\left(E_{\alpha}-\sigma\left(E_{\alpha}\right)\right), E_{\alpha}+\sigma\left(E_{\alpha}\right)\right]_{\left(\alpha, \alpha^{\sigma}\right) \in \bar{\Delta}_{+}\left[\mathfrak{e}_{8}\right] \cap \Delta_{+}\left[\mathfrak{e}_{6}\right]}, \\
\mathfrak{l}_{-}^{\prime} & =\operatorname{Span}\left[i\left(E_{-\alpha}-\sigma\left(E_{-\alpha}\right)\right), E_{-\alpha}+\sigma\left(E_{-\alpha}\right)\right]_{\left(\alpha, \alpha^{\sigma}\right) \in \bar{\Delta}_{+}\left[\mathfrak{e}_{8}\right] \cap \Delta_{+}\left[\mathfrak{e}_{6}\right]}, \\
\mathfrak{m}_{0} & =\operatorname{Span}\left[i\left(E_{\alpha}+E_{-\alpha}\right), E_{\alpha}-E_{-\alpha}\right]_{\alpha \in \Delta_{+}^{(0)}\left[\mathfrak{e}_{8}\right]},
\end{aligned}
$$

where $\Delta_{+}\left[\mathfrak{e}_{6}\right]$ are the $\mathfrak{e}_{6}$-positive roots in the $\mathfrak{e}_{8}$-root system, while

$$
\mathfrak{h}^{\prime}=\mathfrak{h}^{\prime n c} \oplus \mathfrak{h}^{c} ; \mathfrak{h}^{\prime n c}=\operatorname{Span}\left(H_{\epsilon_{1}+\epsilon_{2}-\epsilon_{8}}, H_{\epsilon_{3}}\right) .
$$

The $\mathfrak{s l}(3, \mathbb{R})$ subalgebra commuting with $\mathfrak{e}_{6(-26)}$ has the following form:

$$
\mathfrak{s l}(3, \mathbb{R})=\operatorname{Span}\left[H_{\epsilon_{1}-\epsilon_{2}}, H_{-\epsilon_{1}-\epsilon_{8}}, E_{ \pm \beta_{x}}\right],\left\{\beta_{x}\right\}=\left\{\epsilon_{1}-\epsilon_{2}, \epsilon_{8}+\epsilon_{1}, \epsilon_{8}+\epsilon_{2}\right\},
$$

note that $\beta_{x}^{\sigma}=\beta_{x}$. 


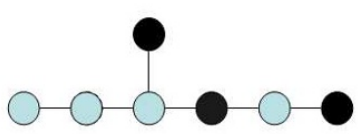

$E_{7(-5)}$

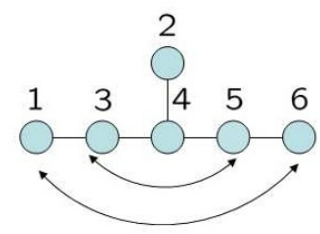

$E_{6(2)}$

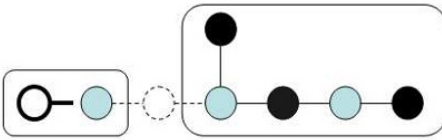

$\mathrm{SL}(3, \mathbb{R}) \times \mathrm{SU}^{*}(6) \subset E_{7(-5)}$

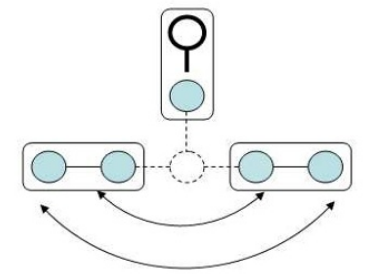

$\mathrm{SL}(3, \mathbb{C}) \times \mathrm{SL}(3, \mathbb{R}) \subset E_{6(2)}$

Figure 4. Embeddings $\mathrm{SL}(3, \mathbb{R}) \times \mathrm{SU}^{*}(6) \subset E_{7(-5)}$ and $\mathrm{SL}(3, \mathbb{C}) \times \mathrm{SL}(3, \mathbb{R}) \subset E_{6(2)}$. The thick circle is, as usual, the opposite of the highest root of the corresponding algebra.

Finally the $\mathfrak{s o}(1,9) \subset \mathfrak{e}_{6(-26)}$ generators read:

$$
\begin{aligned}
\mathfrak{s o}(1,9) & =\mathfrak{h}^{\prime \prime} \oplus \mathfrak{l}_{+}^{\prime \prime} \oplus \mathfrak{l}_{-}^{\prime \prime} \oplus \mathfrak{m}_{0}, \\
\mathfrak{l}_{+}^{\prime \prime} & =\operatorname{Span}\left[i\left(E_{\alpha}-\sigma\left(E_{\alpha}\right)\right), E_{\alpha}+\sigma\left(E_{\alpha}\right)\right]_{\left(\alpha, \alpha^{\sigma}\right) \in \bar{\Delta}_{+}\left[\mathfrak{e}_{8}\right] \cap \Delta_{+}[\mathfrak{s o}(10)]}, \\
\mathfrak{l}_{-}^{\prime \prime} & =\operatorname{Span}\left[i\left(E_{-\alpha}-\sigma\left(E_{-\alpha}\right)\right), E_{-\alpha}+\sigma\left(E_{-\alpha}\right)\right]_{\left(\alpha, \alpha^{\sigma}\right) \in \bar{\Delta}_{+}\left[\mathfrak{e}_{8}\right] \cap \Delta_{+}[\mathfrak{s o}(10)]}, \\
\mathfrak{m}_{0} & =\operatorname{Span}\left[i\left(E_{\alpha}+E_{-\alpha}\right), E_{\alpha}-E_{-\alpha}\right]_{\alpha \in \Delta_{+}^{(0)}\left[\mathfrak{e}_{8}\right]},
\end{aligned}
$$

where $\Delta_{+}[\mathfrak{s o}(10)]$ are the roots of the complex $\mathfrak{s o}(10)$ algebra within $\mathfrak{e}_{8}$-root system, and

$$
\mathfrak{h}^{\prime \prime}=\mathfrak{h}^{\prime \prime n c} \oplus \mathfrak{h}^{c} ; \mathfrak{h}^{\prime \prime n c}=\operatorname{Span}\left(H_{\epsilon_{1}+\epsilon_{2}+\epsilon_{3}-\epsilon_{8}}\right) .
$$

The $\mathfrak{s l}(4, \mathbb{R})$ subalgebra commuting with $\mathfrak{s o}(1,9)$ is described by the following generators:

$$
\mathfrak{s l}(4, \mathbb{R})=\operatorname{Span}\left[H_{\epsilon_{1}-\epsilon_{2}}, H_{\epsilon_{2}-\epsilon_{3}}, H_{-\epsilon_{1}-\epsilon_{8}}, E_{ \pm \beta_{x}}\right],\left\{\beta_{x}\right\}=\left\{\epsilon_{\alpha}-\epsilon_{\beta}, \epsilon_{8}+\epsilon_{\alpha}\right\}_{\alpha<\beta, \alpha, \beta=1,2,3},
$$

By the same token we can prove other embeddings, like $\mathrm{SL}(3, \mathbb{R}) \times \mathrm{SU}^{*}(6) \subset E_{7(-5)}$ and $\mathrm{SL}(3, \mathbb{C}) \times \mathrm{SL}(3, \mathbb{R}) \subset E_{6(2)}$, see figure 4 . In the latter case there is a subtlety which is not apparent from the truncation of the extended Satake diagram: The bottom-right diagram in figure 4 would naively suggest that the roots $\alpha_{1}, \alpha_{3}, \alpha_{5}, \alpha_{6}$ define two commuting $\mathfrak{s l}(3, \mathbb{R})$ subalgebras. This is however not the case since, as represented by the lower arrows, the conjugation $\sigma$ corresponding to the real form $\mathfrak{e}_{6(2)}$ inside the complex $\mathfrak{e}_{6}$, maps $\alpha_{1}$ and $\alpha_{3}$ into $\alpha_{1}^{\sigma}=\alpha_{6}$ and $\alpha_{3}^{\sigma}=\alpha_{5}$, respectively. As a consequence of this the $\mathfrak{e}_{6}$ shift generators corresponding to the two orthogonal $\mathfrak{s l}(3, \mathbb{R})$ root spaces are mixed together in $\sigma$-invariant combinations inside $\mathfrak{e}_{6(2)}$, which make the shift generators of a $\mathfrak{s l}(3, \mathbb{C})$ subalgebra. This subalgebra also contains the two non-compact combinations $H_{\alpha_{1}}+H_{\alpha_{6}}, H_{\alpha_{2}}+H_{\alpha_{5}}$ and the two compact combinations $i\left(H_{\alpha_{1}}-H_{\alpha_{6}}\right), i\left(H_{\alpha_{2}}-H_{\alpha_{5}}\right)$ of the $\mathfrak{e}_{6}$ Cartan generators.

In figure 5 the embeddings $\mathrm{SL}(4, \mathbb{R}) \times \mathrm{SO}(3) \times \mathrm{SO}(1,5) \subset E_{7(-5)}$ and $\mathrm{SL}(2, \mathbb{C}) \times$ $\mathrm{SL}(4, \mathbb{R}) \times \mathrm{SO}(2) \subset E_{6(2)}$ are illustrated. 


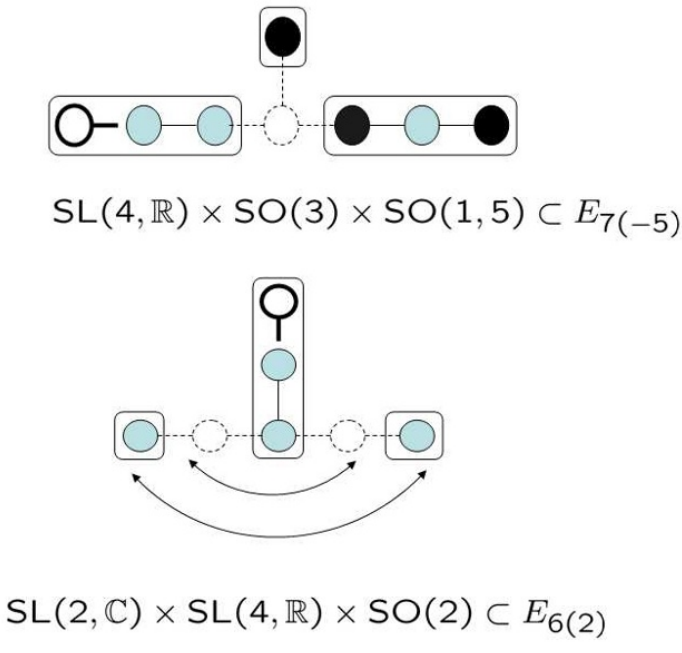

Figure 5. Embeddings $\mathrm{SL}(4, \mathbb{R}) \times \mathrm{SO}(3) \times \mathrm{SO}(1,5) \subset E_{7(-5)}$ and $\mathrm{SL}(2, \mathbb{C}) \times \mathrm{SL}(4, \mathbb{R}) \times \mathrm{SO}(2) \subset$ $E_{6(2)}$.

\section{A.3 General features}

One can generalize the above discussion and show that, as a general feature of the embeddings considered in this work, the $\mathfrak{g}_{N}^{3}$ algebra, and its super-Ehlers subalgebra $\mathfrak{g}_{N}^{D} \oplus \mathfrak{s} \mathfrak{l}(D-2)$ can be written in the forms:

$$
\mathfrak{g}_{N}^{3}=\mathfrak{h} \oplus \mathfrak{l}_{+} \oplus \mathfrak{l}_{-} \oplus \mathfrak{m}_{0} ; \quad \mathfrak{g}_{N}^{D} \oplus \mathfrak{s l}(D-2)=\mathfrak{h} \oplus \hat{\mathfrak{l}}_{+} \oplus \hat{\mathfrak{l}}_{-} \oplus \mathfrak{m}_{0}
$$

Note that, as a consequence of the regularity of the embedding and properties (1.3), (1.4), their Cartan subalgebras

$$
\mathfrak{h}=\mathfrak{h}^{n c} \oplus \mathfrak{h}^{c},
$$

can be chosen to coincide, where $\operatorname{dim}\left(\mathfrak{h}^{n c}\right)$ is the non-compact rank of the two groups. This is implicit in Dynkin's construction of the $\mathfrak{g}_{N}^{D} \oplus \mathfrak{s l}(D-2)$ algebra by truncating the extended diagram of $\mathfrak{g}_{N}^{3}$. Moreover the centralizer of $\mathfrak{h}^{n c}$, which is the compact algebra $\mathfrak{h}^{c} \oplus \mathfrak{m}_{0}$, is common to the two algebras:

$$
\mathfrak{h}^{c} \oplus \mathfrak{m}_{0} \subset \mathfrak{g}_{N}^{3} \bigcap\left[\mathfrak{g}_{N}^{D} \oplus \mathfrak{s l}(D-2)\right] .
$$

For a split (maximally non-compact) $\mathfrak{g}_{N}^{3}, \mathfrak{h}^{c}=\mathfrak{m}_{0}=\varnothing$ and $\alpha^{\sigma}=\alpha$.

The nilpotent spaces $\mathfrak{l}_{ \pm}, \hat{\mathfrak{l}}_{ \pm}$have the form:

$$
\begin{aligned}
& \mathfrak{l}_{ \pm}=\operatorname{Span}\left[i\left(E_{ \pm \alpha}-\sigma\left(E_{ \pm \alpha}\right)\right), E_{ \pm \alpha}+\sigma\left(E_{ \pm \alpha}\right)\right]_{\left(\alpha, \alpha^{\sigma}\right) \in \bar{\Delta}_{+}\left[\mathfrak{g}_{N}^{3}\right]}, \\
& \hat{\mathfrak{l}}_{ \pm}=\operatorname{Span}\left[i\left(E_{ \pm \alpha}-\sigma\left(E_{ \pm \alpha}\right)\right), E_{ \pm \alpha}+\sigma\left(E_{ \pm \alpha}\right)\right]_{\left(\alpha, \alpha^{\sigma}\right) \in \bar{\Delta}_{+}\left[\mathfrak{g}_{N}^{3}\right] \cap \Delta_{+}\left[\mathfrak{g}_{N}^{D} \oplus \mathfrak{s} \mathfrak{l}(D-2)\right]},
\end{aligned}
$$


where, as usual, $\bar{\Delta}_{+}\left[\mathfrak{g}_{N}^{3}\right]$ denotes the set of positive roots of the (complexification of) $\mathfrak{g}_{N}^{3}$ with non-trivial restriction to $\mathfrak{h}^{n c}$, and $\Delta_{+}\left[\mathfrak{g}_{N}^{D} \oplus \mathfrak{s l}(D-2)\right]$ the set of positive roots of the (complexification of) $\mathfrak{g}_{N}^{D} \oplus \mathfrak{s l}(D-2)$, which is a subset of $\Delta_{+}\left[\mathfrak{g}_{N}^{3}\right]$. Thus in general we have:

$$
\hat{\mathfrak{l}}_{ \pm} \subset \mathfrak{l}_{ \pm}
$$

We can then write the coset space as follows:

$$
\mathfrak{g}_{N}^{3} \ominus\left[\mathfrak{g}_{N}^{D} \oplus \mathfrak{s l}(D-2)\right]=\mathfrak{N}^{+} \oplus \mathfrak{N}^{-}
$$

where $\mathfrak{N}^{ \pm}=\mathfrak{l}_{ \pm} \ominus \hat{\mathfrak{l}}_{ \pm}$. Semisimplicity of $\mathfrak{g}_{N}^{3}$ and $\mathfrak{g}_{N}^{D}$ implies that $\operatorname{dim}\left(\mathfrak{l}_{+}\right)=\operatorname{dim}\left(\mathfrak{l}_{-}\right)$and $\operatorname{dim}\left(\hat{\mathfrak{l}}_{+}\right)=\operatorname{dim}\left(\hat{\mathfrak{l}}_{-}\right)$, so that $\operatorname{dim}\left(\mathfrak{N}^{+}\right)=\operatorname{dim}\left(\mathfrak{N}^{-}\right)$. More precisely, in a suitable basis, for each strictly-upper-triangular matrix $M_{+}$representing an element in $\mathfrak{N}^{+}$, its (strictlylower-triangular) hermitian-conjugate $M_{-}=M_{+}^{\dagger}=-\tau\left(M_{+}\right)$represents an element in $\mathfrak{N}^{-}$: The former is given by a generator either of the form $i\left(E_{\alpha}-\sigma\left(E_{\alpha}\right)\right)$ or $E_{\alpha}+\sigma\left(E_{\alpha}\right)$, for some $\alpha \in \bar{\Delta}_{+}\left[\mathfrak{g}_{N}^{3}\right] \ominus \bar{\Delta}_{+}\left[\mathfrak{g}_{N}^{D} \oplus \mathfrak{s l}(D-2)\right]$, the latter will either be $-i\left(E_{-\alpha}-\sigma\left(E_{-\alpha}\right)\right)$ or $E_{-\alpha}+\sigma\left(E_{-\alpha}\right)$, corresponding to the same $\alpha$. Thus if $\left\{L_{\ell}^{+}\right\}, \ell=1, \ldots, \operatorname{dim}\left(\mathfrak{N}^{ \pm}\right)$, is a basis of $\mathfrak{N}^{+},\left\{L_{\ell}^{-}\right\}=\left\{-\tau\left(L_{\ell}^{+}\right)\right\}$is a basis of $\mathfrak{N}^{-}$and we can also write the coset space in the form:

$$
\mathfrak{g}_{N}^{3} \ominus\left[\mathfrak{g}_{N}^{D} \oplus \mathfrak{s l}(D-2)\right]=\mathfrak{N}^{c} \oplus \mathfrak{N}^{n c}
$$

where

$$
\mathfrak{N}^{n c}=\operatorname{Span}\left(L_{\ell}^{+}+L_{\ell}^{-}\right) ; \mathfrak{N}^{c}=\operatorname{Span}\left(L_{\ell}^{+}-L_{\ell}^{-}\right),
$$

which are the eigenspaces of $\tau$ on $\mathfrak{N}^{+} \oplus \mathfrak{N}^{-}$corresponding to the eigenvalues -1 and +1 , respectively. These subspaces define representations with respect to the compact group $\mathrm{SO}(D-2) \times m c s\left(G_{N}^{D}\right)$. With respect to the $G_{N}^{3}$-invariant scalar product on $\mathfrak{g}_{N}^{3}, \mathfrak{N}^{c}$ and $\mathfrak{N}^{n c}$ have negative and positive signatures, respectively. Since

$$
\operatorname{dim}\left(\mathfrak{N}^{c}\right)=\operatorname{dim}\left(\mathfrak{N}^{n c}\right),
$$

the manifold $M_{N}^{D}$ in (5.1) has vanishing character, being

$$
c\left(M_{N}^{D}\right)=\operatorname{dim}\left(\mathfrak{N}^{c}\right)=\operatorname{dim}\left(\mathfrak{N}^{n c}\right)=n c\left(M_{N}^{D}\right),
$$

as also proven in Sect 5.3. We shall come back on this issue in appendix C.

\section{B $\mathfrak{s o}(8,8)$ outer automorphisms and dual subalgebras of $\mathfrak{e}_{8(8)}$}

Consider in the maximal $D=3$ theory the effect of an $\mathrm{O}(8,8)$ "reflection" of the form:

$$
\mathcal{O}_{k}=\left(\begin{array}{cc}
\mathbf{1}_{8}-\mathbf{D}_{k} & \mathbf{D}_{k} \\
\mathbf{D}_{k} & \mathbf{1}_{8}-\mathbf{D}_{k}
\end{array}\right),
$$

where each block is an $8 \times 8$ matrix and $\mathbf{D}_{k}$ is the zero-matrix except for only an odd number $k$ of 1 s along the diagonal. Such transformation, which belongs to the $\mathrm{O}(8)$ subgroup of $\mathrm{O}(8,8)$, is an outer automorphism of the $D_{8}$ algebra whose effect, modulo Weyl 


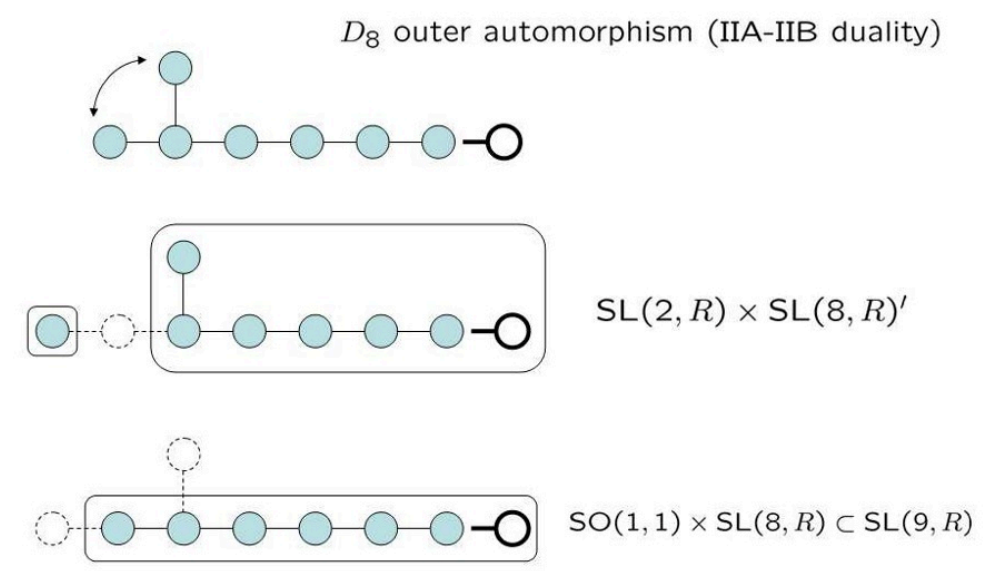

Figure 6. Outer automorphism of the $D_{8}$ subalgebra of $\mathfrak{e}_{8}$ and two inequivalent $\mathfrak{s l}(8, \mathbb{R})$ subalgebras of $\mathfrak{e}_{8(8)}$.

transformations of the same algebra, is to interchange $\alpha_{2}$ with $\alpha_{3}$ in figure 1 . While it is a symmetry of the $D_{8}$ Dynkin diagram, it is not a symmetry of the $\mathfrak{e}_{8(8)}$ one, as it changes the $\mathrm{SO}(8,8)$-chirality of the $\alpha_{1}$ root, which is a $D_{8}$-spinorial weight [25]. In particular this outer automorphism may map inequivalent subalgebras $\mathfrak{g}, \mathfrak{g}^{\prime}$ of $\mathfrak{s o}(8,8)$ into one another. This is the case of subalgebras $\mathfrak{g}$ (and thus $\mathfrak{g}^{\prime}$ ) which are the direct sum of commuting $A_{k^{-}}$ algebras with odd rank $k$. In mathematical language such $d u a l$ subalgebras are said to be linearly equivalent, i.e. in any matrix representation they are equivalent through conjugation by means of a matrix, which is however not necessarily a representation of an $\mathrm{SO}(8,8)$ element, as it is the case for the outer automorphisms. Equivalence therefore implies linear equivalence though the reverse implication is not true. With respect to $\mathfrak{g}$ and $\mathfrak{g}^{\prime}$, a same spinorial representation of $\mathfrak{s o}(8,8)$, and thus the adjoint representation of the whole $\mathfrak{e}_{8(8)}$, will branch differently. They are clearly inequivalent $\mathfrak{e}_{8(8)}$-subalgebras. Examples are given in $[44]: \mathfrak{g}=\mathfrak{s l}(8), \mathfrak{s l}(6) \oplus \mathfrak{s l}(2), \mathfrak{s l}(4) \oplus \mathfrak{s l}(4)$, etc., see Fig 6 .

What has been said for $\mathfrak{s o}(8,8)$ also holds for the $\mathfrak{s o}^{*}(16)$ and $\mathfrak{s o}(16)$ subalgebras of $\mathfrak{e}_{8(8)}$. For instance there are two inequivalent $\mathfrak{u}(8), \mathfrak{u}^{\prime}(8)$ in either $\mathfrak{s o}^{*}(16)$ or $\mathfrak{s o}(16)$. One contains the $R$-symmetry algebras $\mathfrak{s u}(8), \mathfrak{u s p}(8)$, etc. of the higher dimensional parent maximal supergravities, the other dual subalgebras $\mathfrak{s u}^{\prime}(8), \mathfrak{u} \mathfrak{s p}^{\prime}(8)$, etc. which are not contained in the chain of exceptional duality algebras $\mathfrak{e}_{7(7)}, \mathfrak{e}_{6(6)}$ etc.

Let us briefly recall the relation between outer automorphisms of $\mathfrak{s o}(8,8)$ and dualities. Consider the toroidal reduction of the $D=11$ theory down to $D=3$ (in the Einstein frame). The Kaluza-Klein ansatz for the metric reads:

$$
G_{\hat{\mu} \hat{\nu}}^{(11)}=\left(\begin{array}{cc}
e^{2 \xi} g_{\mu \nu}^{(3)}+G_{p q} G_{\mu}^{p} G_{\nu}^{q} & G_{n p} G_{\mu}^{p} \\
G_{m p} G_{\nu}^{p} & G_{m n}
\end{array}\right) ; \xi=-\frac{1}{2} \log \left(\operatorname{det}\left(G_{m n}\right)\right),
$$

where $\hat{\mu}, \hat{\nu}=0, \ldots, 10, \mu, \nu=0,1,2, m, n=3, \ldots, 10$ and the internal metric of $T^{8}$ is 
conveniently written as follows:

$$
\mathbf{G}=\left(G_{m p}\right)=\mathbf{E} \mathbf{E}^{T}=\hat{\mathbf{E}} \mathbf{D}^{2} \hat{\mathbf{E}}^{T},
$$

where $\mathbf{E}=\left(E_{m}{ }^{a}\right)$ is the vielbein of the coset $\mathrm{GL}(8, \mathbb{R}) / \mathrm{SO}(8), a=3, \ldots, 10$, written as the product of a matrix $\hat{\mathbf{E}}$ which only depends on the axionic moduli associated with the off-diagonal components of the metric times the diagonal matrix $\mathbf{D}=\left(D_{m}{ }^{a}\right)=\left(e^{\sigma_{a}} \delta_{m}^{a}\right)$. The exponentials $e^{\sigma_{a}}$ can be viewed as the internal radii $R_{a}$. The bosonic section of the $D=3$ Lagrangian reads: ${ }^{22}$

$$
\begin{aligned}
e^{-1} \mathcal{L}_{3}= & \frac{R}{2}-\frac{1}{2} \partial_{\mu} \vec{h} \cdot \partial^{\mu} \vec{h}-\frac{1}{2} \sum_{a<b} e^{2\left(\sigma_{b}-\sigma_{a}\right)} P_{\mu a}{ }^{b} P^{\mu}{ }_{a}{ }^{b}-\frac{1}{2} \sum_{a} e^{-2\left(\sigma_{a}-\xi\right)} F_{\mu a} F^{\mu}{ }_{a}- \\
& -\frac{1}{4} \sum_{a, b} e^{2\left(\sigma_{a}+\sigma_{b}+\xi\right)} F_{\mu}{ }^{a b} F^{\mu a b}-\frac{1}{12} \sum_{a, b, c} e^{-2\left(\sigma_{a}+\sigma_{b}+\sigma_{c}\right)} F_{\mu a b c} F^{\mu}{ }_{a b c},
\end{aligned}
$$

where $P_{\mu a}^{b} \equiv\left(\hat{\mathbf{E}}^{-1} \partial_{\mu} \hat{\mathbf{E}}\right)_{a}{ }^{b}$ and the dialtonic vector $\vec{h}$ has the following form in the $\left(\epsilon_{i}\right)$ orthonormal basis:

$$
\vec{h}=\sum_{a=3}^{9}\left(\sigma_{a}+\frac{\sigma_{10}}{2}\right) \epsilon_{a-2}+\left(\frac{\sigma_{10}}{2}+\sum_{a=3}^{9} \sigma_{a}\right) \epsilon_{8} .
$$

The field strengths $F_{\mu a}$ and $F_{\mu}^{a b}$ are associated with the scalars $\chi_{n}$ and $\chi^{m n}$ dual in $D=3$ to the vectors $G_{\mu}^{m}$ and $A_{\mu m n}$ respectively, while $F_{\mu a b c}$ is the one pertaining to the scalars $A_{m n p}$. In these conventions, the lower (or upper) internal $\mathrm{SO}(8)$-indices $a, b, c$ of these field strengths are related to the $\operatorname{SL}(8, \mathbb{R})$ indices $m, n, p$ by means of $\hat{\mathbf{E}}$ (or $\hat{\mathbf{E}}^{-1}$ ). For instance:

$$
F_{\mu a b c}=\hat{E}_{a}{ }^{m} \hat{E}_{b}{ }^{n} \hat{E}_{c}^{p} F_{\mu m n p} ; F_{\mu m n p}=\partial_{\mu} A_{m n p} .
$$

The above Lagrangian can also be written in the more compact form:

$$
e^{-1} \mathcal{L}_{3}=\frac{R}{2}-\frac{1}{2} \partial_{\mu} \vec{h} \cdot \partial^{\mu} \vec{h}-\frac{1}{2} \sum_{\alpha \in \Delta_{+}\left[\mathfrak{e}_{8(8)}\right]} e^{-2 \alpha \cdot \vec{h}} \Phi_{\mu}^{(\alpha)} \Phi^{\mu(\alpha)},
$$

where the one-forms $\Phi_{\mu}^{(\alpha)}$ are associated with each of the $\mathfrak{e}_{8(8)}$-positive roots $\alpha$ [46-48]. ${ }^{23}$ It is useful to express the various radial moduli $\sigma_{a}$ in terms of the corresponding fields $\hat{\sigma}_{a}$ in the $D=10$ string frame:

$$
\sigma_{a}=\hat{\sigma}_{a}-\frac{\phi}{3}, a=3, \ldots, 9 ; \sigma_{10}=\frac{2}{3} \phi,
$$

we find:

$$
\vec{h}=\sum_{i=1}^{8} h_{i} \epsilon_{i}=\sum_{a=3}^{9} \hat{\sigma}_{a} \epsilon_{a-2}+\left(-2 \phi+\sum_{a=3}^{9} \hat{\sigma}_{a}\right) \epsilon_{8} .
$$

\footnotetext{
${ }^{22}$ We adopt the mostly plus signature for the metric.

${ }^{23}$ The representation (B.7) of the $D=3$ Lagrangian applies to all $D=3$ supergravities. In the general (non necessarily maximal) case, $\vec{h}$ is a suitable dilaton-dependent vector in the $\mathfrak{h}^{\text {nc }}$ subspace of the Cartan subalgebra of $\mathfrak{g}_{N}^{3}$, while $\alpha$ are the restrictions to $\mathfrak{h}^{n c}$ of the $\mathfrak{g}_{N}^{3}$ positive roots (restricted roots, see [14]).
} 
The outer automorphism $\mathcal{O}_{k}$ in (B.1) has the effect of changing the sign to an odd number of $\epsilon_{a}$, or, equivalently, to their coefficients in $\vec{h}$ :

$$
\epsilon_{i_{\ell}} \rightarrow-\epsilon_{i_{\ell}} ; \ell=1, \ldots, k
$$

To see this let us consider the effect of $\mathcal{O}_{k}$ on the dilatonic part of the coset representative of $\mathrm{O}(8,8) /[\mathrm{O}(8) \times \mathrm{O}(8)]$, which has the following form:

$$
\mathbf{D}(\vec{h})=\left(\begin{array}{cc}
\left(e^{h_{i}} \delta_{i}^{j}\right) & \mathbf{0} \\
\mathbf{0} & \left(e^{-h_{i}} \delta_{i}^{j}\right)
\end{array}\right) .
$$

We see that:

$$
\mathcal{O}_{k}^{-1} \mathbf{D}(\vec{h}) \mathcal{O}_{k}=\mathbf{D}\left(\vec{h}^{\prime}\right),
$$

where $h_{i_{\ell}}^{\prime}=-h_{i_{\ell}}, h_{i \neq i_{\ell}}^{\prime}=h_{i \neq i_{\ell}}, \ell=1, \ldots, k$. If $i_{\ell}$ run between 1 and 7 , this transformation amounts to a $T$-duality along the internal directions $y^{i_{\ell}+2}[25,45]$ :

$$
R_{i_{\ell}+2}^{\prime}=e^{\hat{\sigma}_{i_{\ell}+2}^{\prime}}=e^{-\hat{\sigma}_{i_{\ell}+2}}=\frac{1}{R_{i_{\ell}+2}} ; \phi^{\prime}=\phi-\sum_{\ell=1}^{k} \hat{\sigma}_{i_{\ell}+2} .
$$

These transformations map type IIA into type IIB theory. If $k=1$ and $i_{\ell}=8$ then there is an $S$-duality involved: $\hat{\sigma}_{i}^{\prime}=\hat{\sigma}_{i}$ and $\phi^{\prime}=-\phi+\sum_{a=3}^{9} \hat{\sigma}_{a}$.

Instead of considering inequivalent T-dual subalgebras $\mathfrak{g}, \mathfrak{g}^{\prime} \subset \mathfrak{s o}(8,8)$ within a same $\mathfrak{e}_{8(8)}$ algebra, we may adopt an equivalent point of view and consider a same subalgebra $\mathfrak{g} \subset \mathfrak{s o}(8,8)$ within two $\mathfrak{e}_{8(8)}$ algebras, called in $[25] \mathfrak{e}_{8(8)}^{+}$and $\mathfrak{e}_{8(8)}^{-},{ }^{24}$ defined respectively by completing the $\mathfrak{s o}(8,8)$ Dynkin diagram with spinorial weights of different chiralities, namely attaching the weight $\alpha_{1}$ to $\alpha_{3}$, as in figure 1 , or a weight $\alpha_{1}^{\prime}$ to $\alpha_{2}$, defined as follows:

$$
\alpha_{1}=-\frac{1}{2}\left(\sum_{i=1}^{8} \epsilon_{i}\right)+\epsilon_{7}+\epsilon_{8} \stackrel{\text { T-duality along } y^{9}}{\longrightarrow} \alpha_{1}^{\prime}=-\frac{1}{2}\left(\sum_{i=1}^{8} \epsilon_{i}\right)+\epsilon_{8} .
$$

This is useful if, for instance, we fix the $\mathfrak{g}=\mathfrak{g l}(8, \mathbb{R}) \subset \mathfrak{s o}(8,8)$ group to be the same in the type IIA and type IIB settings. Then the different $\mathfrak{g r}(8, \mathbb{R})$-weights defining the dimensionally reduced type IIA and type IIB forms are obtained by branching the adjoint representations of $\mathfrak{e}_{8(8)}^{+}$and $\mathfrak{e}_{8(8)}^{-}$, respectively, with respect to the common $\mathfrak{g l}(8, \mathbb{R}),[25]$.

The doubling of the equivalence classes inside a $D_{n}$ algebra into dual pairs, discussed above, does not occur if the subalgebra is the sum of commuting algebras in the case in which either all of them are of type $A_{k}$ with even rank $k$, or at least one of them is of type $D$ [42]. This is consistent with the fact observed in subsection 2.1, that the $\mathrm{SL}(7, \mathbb{R})$ $D=9$ Ehlers subgroups of $\mathrm{SO}(8,8)$ which pertain to the type IIA and IIB descriptions are equivalent. The same rule guarantees that, in $D=6$, the $\mathrm{SO}(5,5) \times \mathrm{SL}(4, \mathbb{R})$ subgroups of $\mathrm{SO}(8,8)$ in the type IIA and IIB settings, are equivalent.

\footnotetext{
${ }^{24}$ Actually in [25] only the $D=4$ theory was considered, the $T$-duality group being $\mathrm{O}(6,6)$ in this case, and the algebras $\mathfrak{e}_{7(7)}^{ \pm}$defined.
} 


\section{Poincaré duality and level decomposition}

Consider now the branching of the adjoint representation of $\mathfrak{g}_{N}^{3}$ with respect to $\operatorname{SL}(D-$ 2) $\times G_{N}^{D}$ :

$$
\begin{aligned}
\operatorname{Adj}_{G_{N}^{3}} & \rightarrow\left(\operatorname{Adj}_{\operatorname{SL}(D-2)}, \mathbf{1}\right) \oplus\left(\mathbf{1}, \mathbf{A d j}_{G_{N}^{D}}\right) \bigoplus_{d} \mathfrak{N}_{d}, \\
\mathfrak{N}_{d} & =\left[\left(\Lambda^{d}, \mathcal{R}_{d}\right) \oplus\left(* \Lambda^{d}, \mathcal{R}_{d}^{\prime}\right)\right]
\end{aligned}
$$

where it is understood that if $\left(\Lambda^{d}, \mathcal{R}_{d}\right)=\left(* \Lambda^{d}, \mathcal{R}_{d}^{\prime}\right)$, they are counted just once in $\mathfrak{N}_{d}$. In light of our discussion in appendix A.3, we can write the coset space as the carrier of a representation $\bigoplus_{d} \mathfrak{N}_{d}$, namely rewrite eq. (A.26) as follows:

$$
\mathfrak{g}_{N}^{3} \ominus\left(\mathfrak{g}_{N}^{D} \oplus \mathfrak{s l}(D-2)\right)=\mathfrak{N}^{+} \oplus \mathfrak{N}^{-}=\bigoplus_{d} \mathfrak{N}_{d} .
$$

In fact each subspace $\mathfrak{N}_{d}$ splits into conjugate nilpotent subalgebras as follows:

$$
\mathfrak{N}_{d}=\mathfrak{N}_{d}^{+} \oplus \mathfrak{N}_{d}^{-}, \mathfrak{N}_{d}^{+}=\mathfrak{N}_{d} \bigcap \mathfrak{N}^{+}, \mathfrak{N}_{d}^{-}=\mathfrak{N}_{d} \bigcap \mathfrak{N}^{-}=\tau\left(\mathfrak{N}_{d}^{+}\right)
$$

this being a consequence of the property: $\tau\left(\mathfrak{N}_{d}\right)=\mathfrak{N}_{d}$. Each nilpotent subalgebra $\mathfrak{N}_{d}^{+}$ or $\mathfrak{N}_{d}^{-}$separately defines a representation with respect to (the adjoint action of) $G_{N}^{D}$ and the subgroup $\mathrm{GL}(D-3) \subset \mathrm{SL}(D-2)$, though not with respect to $\operatorname{SL}(D-2)$ itself. We can decompose each space $\mathfrak{N}_{d}$ into eigenspaces of the Cartan involution $\tau$, consisting of compact and non-compact generators:

$$
\mathfrak{N}_{d}=\mathfrak{N}_{d}^{c} \oplus \mathfrak{N}_{d}^{n c}, \mathfrak{N}_{d}^{c}=\mathfrak{N}^{c} \bigcap \mathfrak{N}_{d}, \mathfrak{N}_{d}^{n c}=\mathfrak{N}^{n c} \bigcap \mathfrak{N}_{d} .
$$

These subspaces define representations with respect to the compact group $\mathrm{SO}(D-2) \times$ $\operatorname{mcs}\left(G_{N}^{D}\right)$ and, moreover

$$
\operatorname{dim}\left(\mathfrak{N}_{d}^{c}\right)=\operatorname{dim}\left(\mathfrak{N}_{d}^{n c}\right) .
$$

For the sake of simplicity, let us consider a split (maximally non-compact) $\mathfrak{g}_{N}^{3}$. Then each $\mathfrak{N}_{d}$ will be generated by shift operators corresponding to a certain set of positive roots $\alpha^{(d)}$ and their negatives:

$$
\mathfrak{N}_{d}=\operatorname{Span}\left(E_{\alpha^{(d)}}, E_{-\alpha^{(d)}}\right)_{\alpha^{(d)} \in \Delta_{+}\left[\mathfrak{g}_{N}^{3}\right]},
$$

and the conjugate nilpotent subalgebras are $\mathfrak{N}_{d}^{+}=\operatorname{Span}\left(E_{\alpha^{(d)}}\right)$ and $\mathfrak{N}_{d}^{-}=\operatorname{Span}\left(E_{-\alpha^{(d)}}\right)$. The eigenspaces $\mathfrak{N}_{d}^{n c}, \mathfrak{N}_{d}^{c}$ of the Cartan involution, consisting of compact and non-compact generators read:

$$
\mathfrak{N}_{d}^{c}=\operatorname{Span}\left(E_{\alpha^{(d)}}-E_{-\alpha^{(d)}}\right)_{\alpha^{(d)} \in \Delta_{+}\left[\mathfrak{g}_{N}^{3}\right]} ; \quad \mathfrak{N}_{d}^{n c}=\operatorname{Span}\left(E_{\alpha^{(d)}}+E_{-\alpha^{(d)}}\right)_{\alpha^{(d)} \in \Delta_{+}\left[\mathfrak{g}_{N}^{3}\right]} .
$$

Each positive root $\alpha^{(d)}$ corresponds to a $D=3$ scalar field in the Lagrangian (B.7). For a given $d$ the roots $\alpha^{(d)}$ are defined by the level decomposition of the $\mathfrak{g}_{N}^{3}$-roots with respect to the root which is truncated out of its extended diagram in order to define the $\mathfrak{g}_{N}^{D} \oplus \mathfrak{s l}(D-2)$ subdiagram. ${ }^{25}$

\footnotetext{
${ }^{25}$ In the non-split case, one should consider the level decomposition of the restricted roots. Level decompositions are a common procedure in the $E_{10}$ and $E_{11}$ approaches to maximal supergravity [49, 50].
} 
Let us illustrate this procedure in the maximal theory. As shown in appendix A, the $\mathfrak{e}_{11-D(11-D)} \times \mathfrak{s l}(D-2)$ diagram is obtained by deleting from the $\mathfrak{e}_{8(8)}$-extended Dynkin diagram the root $\alpha_{12-D}$. The $\mathfrak{s l}(D-2)$ subalgebra is defined by the simple roots $\alpha_{13-D}, \ldots, \alpha_{8},-\psi, \psi=\epsilon_{1}+\epsilon_{8}$ being the $\mathfrak{e}_{8(8)}$ highest root, while its $\mathfrak{g l}(D-3)$ subalgebra only by the roots $\alpha_{13-D}, \ldots, \alpha_{8}$. Writing a generic $\mathfrak{e}_{8(8)}$ positive root in the simple root basis:

$$
\alpha=\sum_{i=1}^{8} n_{i} \alpha_{i},
$$

the positive integer $n_{i}$ defines the level of $\alpha$ with respect to $\alpha_{i}$. Let us consider the leveldecomposition with respect to the root $\alpha_{12-D}$ for dimensions $D<9$, namely the values of $n_{12-D}$ defining the roots $\alpha^{(d)} .^{26}$

$\boldsymbol{D}=4$. In the case of $D=4$ we have 63 roots with $n_{8}=0$, corresponding to the $\mathfrak{e}_{7(7)^{-}}$ positive roots. The level $n_{8}=1$ roots are 56 and are the $\alpha^{(1)}$-roots whose shift generators $E_{ \pm \alpha^{(1)}}$ define the carrier space of the $\mathfrak{N}_{d=1}=(\mathbf{1}, \mathbf{5 6})$ representation. The level $n_{8}=2$ root defines, with its negative, the shift generators in the quotient $\mathfrak{s l}(D-2) \ominus \mathfrak{g l}(D-3)=$ $\mathfrak{s l}(2) \ominus \mathfrak{g l}(1)$, which are the two shift generators of the Ehlers group.

$\boldsymbol{D}=\mathbf{5}$. Consider now the $D=5$ case. There are 37 level- $n_{7}=0$ roots corresponding to the positive roots of $\mathfrak{e}_{6(6)} \oplus \mathfrak{g l}(2)$. The level- $n_{7}=1$ roots are 54 and define in $\mathfrak{N}_{d=1}^{+}$a subspace in the $(\mathbf{2}, \mathbf{2 7})$-representation of $\mathrm{SL}(D-3) \times E_{6(6)}=\mathrm{SL}(2) \times E_{6(6)}$, while the 27 level- $n_{7}=2$ roots define a subspace in the $\left(\mathbf{1}, \mathbf{2} \mathbf{7}^{\prime}\right)$ with respect to the same group. The space $\mathfrak{N}_{d=1}^{-}$will be the carrier of the conjugate representations. Together, the level $n_{7}=1,2$ roots and their negatives define the space $\mathfrak{N}_{d=1}=\mathfrak{N}_{d=1}^{+} \oplus \mathfrak{N}_{d=1}^{-}=(\mathbf{3}, \mathbf{2 7}) \oplus\left(\mathbf{3}^{\prime}, \mathbf{2} \mathbf{7}^{\prime}\right)$, and are collectively denoted by $\alpha^{(1)}$. Finally the 2 level $-n_{7}=3$ roots, with their negative, define the generators of the coset $\mathfrak{s l}(D-2) \ominus \mathfrak{g l}(D-3)=\mathfrak{s l}(3) \ominus \mathfrak{g l}(2)$.

$\boldsymbol{D}=\mathbf{6}$. As far as the $D=6$ case is concerned, the 23 level- $n_{6}=0$ roots are positive roots of $\mathfrak{g l}(3) \oplus \mathfrak{s o}(5,5)$, while the 48 level- $n_{6}=1$ and the 16 level- $n_{6}=3$ roots define generators in $\mathfrak{N}_{d=1}^{+}$transforming in the $(\mathbf{3}, \mathbf{1 6})$ and $\left(\mathbf{1}, \mathbf{1 6}^{\prime}\right)$ of $\mathrm{SL}(D-3) \times \mathrm{SO}(5,5)=\mathrm{SL}(3) \times \mathrm{SO}(5,5)$, respectively. These are the $\alpha^{(1)}$ roots, which, together with their negatives, define the $\mathfrak{N}_{d=1}=(\mathbf{4}, \mathbf{1 6}) \oplus\left(\mathbf{4}^{\prime}, \mathbf{1 6}^{\prime}\right)$ space. The roots $\alpha^{(2)}(d=2)$ are 30 and have $n_{6}=2$. The corresponding space $\mathfrak{N}_{d=2}^{+}$is the carrier of a $(\mathbf{3}, \mathbf{1 0})$ representation of $\mathrm{SL}(3) \times \mathrm{SO}(5,5)$, while $E_{ \pm \alpha^{(2)}}$ generate the $\mathfrak{N}_{d=2}=(\mathbf{6}, \mathbf{1 0})$. Finally the 3 level- $n_{6}=4$ roots, with their negative, define the generators of the coset $\mathfrak{s l}(D-2) \ominus \mathfrak{g l}(D-3)=\mathfrak{s l}(4) \ominus \mathfrak{g l}(3)$.

A similar pattern occurs in the higher- $D$ cases.

\footnotetext{
${ }^{26}$ More precisely, the level $n^{i}$ is the grading of the generator $E_{\alpha}$ with respect to the $\mathrm{SO}(1,1)$ generator $H_{\lambda^{i}}$ (i.e. $\left.\left[H_{\lambda^{i}}, E_{\alpha}\right]=n^{i} E_{\alpha}\right), \lambda^{i}$ being the $\mathfrak{g}_{N}^{3}$ simple weights. The level decomposition is defined by the Cartan generator which is orthogonal to the Cartan subalgebra of $\mathfrak{g}_{N}^{D} \oplus \mathfrak{g l}(D-3)$ (and therefore commutes with $\left.\mathfrak{g}_{N}^{D} \oplus \mathfrak{g l}(D-3)\right)$. In the maximal theory, for $D<9$, the relevant Cartan generator is $H_{\lambda^{12-D}}$ and thus the level to consider is $n_{12-D}$. For $D=9$ the generator is $H_{\lambda^{2}}+H_{\lambda^{3}}$ and so we shall consider the decomposition with respect to the integer $n=n_{2}+n_{3}$. In the type IIA $D=10$ description, the generator is $H_{\lambda^{1}}+2 H_{\lambda^{2}}$ and the decomposition will be effected with respect to $n=n_{1}+2 n_{2}$.
} 
$\boldsymbol{D}=7$. For $D=7$, we have 16 roots with $n_{5}=0$ which are the roots of $\mathfrak{g l}(4) \oplus \mathfrak{s l}(5)$. The $40 n_{5}=1$ and the $10 n_{5}=4$ roots define subspaces of $\mathfrak{N}_{d=1}^{+}$in the $\left(\mathbf{4}, \mathbf{1 0}^{\prime}\right)$ and $(\mathbf{1}, \mathbf{1 0})$ of $\mathrm{SL}(D-3) \times \mathrm{SL}(5)=\mathrm{SL}(4) \times \mathrm{SL}(5)$, respectively. These are the $\alpha^{(1)}$-roots and the space $\mathfrak{N}_{d=1}=\mathfrak{N}_{d=1}^{+} \oplus \mathfrak{N}_{d=1}^{-}$is the carrier of the representation $\left(\mathbf{5}, \mathbf{1 0}^{\prime}\right) \oplus\left(\mathbf{5}^{\prime}, \mathbf{1 0}\right)$ of $\mathrm{SL}(5) \times \mathrm{SL}(5)$. The $\alpha^{(2)}$-roots consist in the 30 level- $n_{5}=2$ and the 20 level- $n_{5}=3$ roots defining the representations $(\mathbf{6}, \mathbf{5})$ and $\left(\mathbf{4}^{\prime}, \mathbf{5}^{\prime}\right)$ of $\mathrm{SL}(4) \times \mathrm{SL}(5)$ in $\mathfrak{N}_{d=2}^{+}$, respectively. The space $\mathfrak{N}_{d=2}=\mathfrak{N}_{d=2}^{+} \oplus \mathfrak{N}_{d=2}^{-}$then defines the representation $(\mathbf{1 0}, \mathbf{5}) \oplus\left(\mathbf{1 0}^{\prime}, \mathbf{5}^{\prime}\right)$ of $\mathrm{SL}(5) \times \mathrm{SL}(5)$. Finally the 4 level- $n_{5}=5$ roots, with their negative, define the generators of the coset $\mathfrak{s l}(D-2) \ominus \mathfrak{g l}(D-3)=\mathfrak{s l}(5) \ominus \mathfrak{g l}(4)$.

$\boldsymbol{D}=\mathbf{8}$. In the $D=8$ case the $14 n_{4}=0$ roots are the positive roots of $\mathfrak{g l}(5) \oplus \mathfrak{s l}(2) \oplus \mathfrak{s l}(3)$. The $\alpha^{(1)}$ s consist of the $30 n_{4}=1$ and the $6 n_{4}=5$ roots defining the $\mathrm{SL}(5) \times \mathrm{SL}(2) \times \mathrm{SL}(3)$ representations $\left(\mathbf{5}, \mathbf{2}, \mathbf{3}^{\prime}\right) \oplus(\mathbf{1}, \mathbf{2}, \mathbf{3})$ in $\mathfrak{N}_{d=1}^{+}$which, together with the conjugate representations in $\mathfrak{N}_{d=1}^{-}$, complete the $\mathfrak{N}_{d=1}=\left(\mathbf{6}, \mathbf{2}, \mathbf{3}^{\prime}\right) \oplus\left(\mathbf{6}^{\prime}, \mathbf{2}, \mathbf{3}\right)$ of $\mathrm{SL}(6) \times \mathrm{SL}(2) \times \mathrm{SL}(3)$. The $\alpha^{(2)}$ s are defined by the $30 n_{4}=2$ and $15 n_{4}=4$ roots, corresponding to the representation $(\mathbf{1 0}, \mathbf{1}, \mathbf{3}) \oplus\left(\mathbf{5}^{\prime}, \mathbf{1}, \mathbf{3}^{\prime}\right)$ in $\mathfrak{N}_{d=2}^{+}$, so that $\mathfrak{N}_{d=2}=\mathfrak{N}_{d=2}^{+} \oplus \mathfrak{N}_{d=2}^{-}=(\mathbf{1 5}, \mathbf{1}, \mathbf{3}) \oplus\left(\mathbf{1} \mathbf{5}^{\prime}, \mathbf{1}, \mathbf{3}^{\prime}\right)$ of $\mathrm{SL}(6) \times \mathrm{SL}(2) \times \mathrm{SL}(3)$. The $\alpha^{(3)}$ roots are the 20 ones with $n_{4}=3$. They define the $\mathfrak{N}_{d=3}^{+}$ space in the $(\mathbf{1 0}, \mathbf{2}, \mathbf{1})$ of $\mathrm{SL}(5) \times \mathrm{SL}(2) \times \mathrm{SL}(3)$ which, together with $\mathfrak{N}_{d=3}^{-}$, complete the $\mathfrak{N}_{d=3}=(\mathbf{2 0}, \mathbf{2}, \mathbf{1})$ of $\mathrm{SL}(6) \times \mathrm{SL}(2) \times \mathrm{SL}(3)$. The remaining 5 roots with $n_{4}=6$, with their negative, define the generators of the coset $\mathfrak{s l}(D-2) \ominus \mathfrak{g l}(D-3)=\mathfrak{s l}(6) \ominus \mathfrak{g l}(5)$.

$\boldsymbol{D}=\mathbf{9}$. The same analysis applies to $D=9$, although in this case we shall consider the $\operatorname{sum} n=n_{2}+n_{3}$. There are 16 roots with $n=0$, which are the positive roots of the algebra $\mathfrak{g l}(6) \oplus \mathfrak{g l}(2)$. The $\alpha^{(1)}$ roots consist of the 18 with $n=1$ and the 3 with $n=6$, defining the $\mathrm{SL}(6) \times \mathrm{GL}(2)$-representations $\left(\mathbf{6}, \mathbf{2}_{+3}+\mathbf{1}_{-4}\right) \oplus\left(\mathbf{1}, \mathbf{2}_{-3}+\mathbf{1}_{+4}\right)$ in $\mathfrak{N}_{d=1}^{+}$which, together with the conjugate representations in $\mathfrak{N}_{d=1}^{-}$, complete the $\mathfrak{N}_{d=1}=\left(\mathbf{7}, \mathbf{2}_{+3}+\mathbf{1}_{-4}\right) \oplus\left(\mathbf{7}^{\prime}, \mathbf{2}_{-3}+\mathbf{1}_{+4}\right)$ of $\mathrm{SL}(7) \times \mathrm{GL}(2)$. The $\alpha^{(2)}$ s are the 30 roots with $n=2$ and the 12 with $n=5$ defining the $\mathrm{SL}(6) \times \mathrm{GL}(2)$-representations $\left(\mathbf{1 5}, \mathbf{2}_{-1}\right) \oplus\left(\mathbf{6}^{\prime}, \mathbf{2}_{+1}\right)$ in $\mathfrak{N}_{d=2}^{+}$, so that $\mathfrak{N}_{d=2}=\mathfrak{N}_{d=2}^{+} \oplus \mathfrak{N}_{d=2}^{-}=$ $\left(\mathbf{2 1}, \mathbf{2}_{-1}\right) \oplus\left(\mathbf{2 1}^{\prime}, \mathbf{2}_{+1}\right)$. Next we have to consider the $20 n=3$ and the $15 n=4$ roots which make the $\alpha^{(3)}$ and define the $\mathrm{SL}(6) \times \mathrm{GL}(2)$-representations $\left(\mathbf{2 0}, \mathbf{1}_{+2}\right) \oplus\left(\mathbf{1 5}^{\prime}, \mathbf{1}_{-2}\right)$ in $\mathfrak{N}_{d=3}^{+}$, so that $\mathfrak{N}_{d=3}=\mathfrak{N}_{d=3}^{+} \oplus \mathfrak{N}_{d=3}^{-}=\left(\mathbf{3 5}, \mathbf{1}_{+2}\right) \oplus\left(\mathbf{3 5}^{\prime}, \mathbf{1}_{-2}\right)$. Finally the $6 n=7$ roots, with their negative, define the generators of the coset $\mathfrak{s l}(D-2) \ominus \mathfrak{g l}(D-3)=\mathfrak{s l}(7) \ominus \mathfrak{g l}(6)$.

$\boldsymbol{D}=\mathbf{1 0}$, IIB. In the $D=10$ case we have to distinguish between the type IIA and IIB theories. In the type IIB setting we need consider the level $n_{3}$ with respect to $\alpha_{3}$. The 22 roots with $n_{3}=0$ are the positive roots of $\mathfrak{g l}(7) \oplus \mathfrak{s l}(2), \mathfrak{s l}(2)$ being the $U$-duality group. In this case we only have $d=2,4$. The $\alpha^{(2)}$ s consist of the $42 n_{3}=1$ and the $14 n_{3}=3$ defining the $\mathrm{SL}(7) \times \mathrm{SL}(2)$-representations $(\mathbf{2 1}, \mathbf{2}) \oplus\left(\mathbf{7}^{\prime}, \mathbf{2}\right)$ in $\mathfrak{N}_{d=2}^{+}$, so that $\mathfrak{N}_{d=2}=\mathfrak{N}_{d=2}^{+} \oplus \mathfrak{N}_{d=2}^{-}=(\mathbf{2 8}, \mathbf{2}) \oplus\left(\mathbf{2 8}^{\prime}, \mathbf{2}\right)$ of $\mathrm{SL}(8) \times \mathrm{SL}(2)$. Next we have the 35 roots with $n_{3}=2$, which are the $\alpha^{(4)}$ s and define the $(\mathbf{3 5}, \mathbf{1})$ of $\mathrm{SL}(7) \times \mathrm{SL}(2)$ in $\mathfrak{N}_{d=4}^{+}$, so that $\mathfrak{N}_{d=4}=\mathfrak{N}_{d=4}^{+} \oplus \mathfrak{N}_{d=4}^{-}=(\mathbf{7 0}, \mathbf{1})$. There are $7 n_{3}=4$ roots which, with their negative, define the generators of the coset $\mathfrak{s l}(D-2) \ominus \mathfrak{g l}(D-3)=\mathfrak{s l}(8) \ominus \mathfrak{g l}(7)$.

$D=10$, IIA. As far as the type IIA description is concerned, the level to consider for the decomposition is the sum $n=n_{1}+2 n_{2}$. In this case we only have $d=1,2,3$. There are 
$21 n=0$ roots which are the positive roots of $\mathfrak{g l}(7) \oplus \mathfrak{s o}(1,1), \mathfrak{s o}(1,1)$ being the $U$-duality algebra. The $\alpha^{(1)}$ roots consist of the 7 roots with $n=1$ and the single $n=7$ root defining the $\mathrm{SL}(7) \times \mathrm{SO}(1,1)$-representation $\mathbf{7}_{+3} \oplus \mathbf{1}_{-3}$ in $\mathfrak{N}_{d=1}^{+}$which, together with the conjugate representations in $\mathfrak{N}_{d=1}^{-}$, complete the $\mathfrak{N}_{d=1}=\boldsymbol{8}_{+3} \oplus \boldsymbol{8}_{-3}$ of $\mathrm{SL}(8) \times \mathrm{SO}(1,1)$. Next we consider the 21 roots with $n=2$ and the 7 with $n=6$, whose shift operators generating $\mathfrak{N}_{d=2}^{+}$transform in the $\mathbf{2 1}_{-2} \oplus \mathbf{7}_{+2}^{\prime}$ with respect to $\mathrm{SL}(7) \times \mathrm{SO}(1,1)$. These roots define then the $\alpha^{(2)}$ and $\mathfrak{N}_{d=2}=\mathfrak{N}_{d=2}^{+} \oplus \mathfrak{N}_{d=2}^{-}=\mathbf{2 8}_{-2} \oplus \mathbf{2 8}_{+2}^{\prime}$ of $\mathrm{SL}(8) \times \mathrm{SO}(1,1)$. The $\alpha^{(3)} \mathrm{s}$ consist of the $35 n=3$ and the $21 n=6$ roots defining the $\mathrm{SL}(7) \times \mathrm{SO}(1,1)$-representation $\mathbf{3 5}_{+1} \oplus \mathbf{2} \mathbf{1}_{-1}^{\prime}$ in $\mathfrak{N}_{d=3}^{+}$which, together with the conjugate representations in $\mathfrak{N}_{d=3}^{-}$, complete the $\mathfrak{N}_{d=3}=\mathbf{5 6}_{+1} \oplus \mathbf{5} \mathbf{6}_{-1}^{\prime}$. There are no roots with $n=5$ while those with $n=8$ are 7 and, with their negative, define the generators of the coset $\mathfrak{s l}(D-2) \ominus \mathfrak{g l}(D-3)=\mathfrak{s l}(8) \ominus \mathfrak{g l}(7)$.

$\boldsymbol{D}=\mathbf{1 1}$. We end this analysis with the $D=11$ case discussed in the previous section. The relevant level decomposition is with respect to the root $\alpha_{2}$. With $n_{2}=0$ we have the positive roots of $\mathfrak{g l}(8)$. In this case we only have $d=3$ and the $\alpha^{(3)}$-roots consist of the $56 n_{2}=1$ and the $28 n_{2}=2$ ones defining the $\mathrm{SL}(8) \times \mathrm{SO}(1,1)$-representation $\mathbf{5 6}_{+1} \oplus \mathbf{2 8}_{+2}^{\prime}$ in $\mathfrak{N}_{d=3}^{+}$which, together with the conjugate representations in $\mathfrak{N}_{d=3}^{-}$, completes the $\mathfrak{N}_{d=3}=\mathbf{8 4} \oplus \mathbf{8 4}^{\prime}$. Finally the $8 n_{2}=3$ roots, with their negative, define the generators of the coset $\mathfrak{s l}(D-2) \ominus \mathfrak{g l}(D-3)=\mathfrak{s l}(9) \ominus \mathfrak{g l}(8)$.

Open Access. This article is distributed under the terms of the Creative Commons Attribution License which permits any use, distribution and reproduction in any medium, provided the original author(s) and source are credited.

\section{References}

[1] M. Goroff and J.H. Schwarz, D-dimensional gravity in the light cone gauge, Phys. Lett. B 127 (1983) 61 [INSPIRE].

[2] E. Cremmer and B. Julia, The $N=8$ Supergravity Theory. 1. The Lagrangian, Phys. Lett. B 80 (1978) 48 [INSPIRE].

[3] E. Cremmer and B. Julia, The SO(8) Supergravity, Nucl. Phys. B 159 (1979) 141 [InSPIRE].

[4] C. Hull and P. Townsend, Unity of superstring dualities, Nucl. Phys. B 438 (1995) 109 [hep-th/9410167] [INSPIRE].

[5] E. Cremmer, B. Julia, H. Lü and C. Pope, Higher dimensional origin of $D=3$ coset symmetries, hep-th/9909099 [INSPIRE].

[6] H. Lü and C. Pope, P-brane solitons in maximal supergravities, Nucl. Phys. B 465 (1996) 127 [hep-th/9512012] [INSPIRE].

[7] N. Marcus and J.H. Schwarz, Three-Dimensional Supergravity Theories, Nucl. Phys. B 228 (1983) 145 [inSPIRE].

[8] E. Cremmer, Supergravities in 5 Dimensions, in Superspace and Supergravity, S.W. Hawking and M. Rocek eds., Cambridge University Press, Cambridge, U.K. (1981).

[9] B. Julia, Group Disintegrations, in Superspace and Supergravity, S.W. Hawking and M. Rocek eds., Cambridge University Press, Cambridge, U.K. (1981). 
[10] S. Ananth, L. Brink and P. Ramond, Eleven-dimensional supergravity in light-cone superspace, JHEP 05 (2005) 003 [hep-th/0501079] [INSPIRE].

[11] L. Brink, S.-S. Kim and P. Ramond, E E(8) in Light Cone Superspace, JHEP 07 (2008) 113 [arXiv:0804.4300] [INSPIRE].

[12] L. Brink, S.-S. Kim and P. Ramond, $E_{7(7)}$ on the Light Cone, JHEP 06 (2008) 034 [arXiv: 0801.2993] [INSPIRE].

[13] B. de Wit, A. Tollsten and H. Nicolai, Locally supersymmetric $D=3$ nonlinear $\sigma$-models, Nucl. Phys. B 392 (1993) 3 [hep-th/9208074] [InSPIRE].

[14] S. Helgason, Differential Geometry, Lie Groups and Symmetric Spaces, Academic Press, New York, U.S.A. (1978).

[15] R. Gilmore, Lie Groups, Lie Algebras, and Some of Their Applications, Dover Publications (2006).

[16] A. Keurentjes, The Group theory of oxidation, Nucl. Phys. B 658 (2003) 303 [hep-th/0210178] [INSPIRE].

[17] A. Keurentjes, The Group theory of oxidation 2: Cosets of nonsplit groups, Nucl. Phys. B 658 (2003) 348 [hep-th/0212024] [INSPIRE].

[18] N. Marcus, A. Sagnotti and J.H. Schwarz, Infinite symmetry algebras of extended supergravity theories, Nucl. Phys. B 243 (1984) 335 [INSPIRE].

[19] M. Günaydin, G. Sierra and P. Townsend, Exceptional Supergravity Theories and the MAGIC Square, Phys. Lett. B 133 (1983) 72 [InSPIRE].

[20] M. Günaydin, G. Sierra and P. Townsend, The Geometry of $N=2$ Maxwell-Einstein Supergravity and Jordan Algebras, Nucl. Phys. B 242 (1984) 244 [INSPIRE].

[21] M. Günaydin, Lectures on Spectrum Generating Symmetries and U-duality in Supergravity, Extremal Black Holes, Quantum Attractors and Harmonic Superspace, arXiv:0908.0374 [INSPIRE].

[22] P. Truini, Exceptional Lie Algebras, SU(3) and Jordan Pairs, arXiv:1112.1258 [INSPIRE].

[23] W. Nahm, Supersymmetries and their Representations, Nucl. Phys. B 135 (1978) 149 [INSPIRE].

[24] E. Cremmer, B. Julia and J. Scherk, Supergravity Theory in Eleven-Dimensions, Phys. Lett. B 76 (1978) 409 [INSPIRE].

[25] M. Bertolini and M. Trigiante, Regular RR and NS-NS BPS black holes, Int. J. Mod. Phys. A 15 (2000) 5017 [hep-th/9910237] [INSPIRE].

[26] R. D'Auria, S. Ferrara, M. Lledó and V. Varadarajan, Spinor algebras, J. Geom. Phys. 40 (2001) 101 [hep-th/0010124] [INSPIRE].

[27] S. Ferrara, C.A. Savoy and B. Zumino, General massive multiplets in extended supersymmetry, Phys. Lett. B 100 (1981) 393 [INSPIRE].

[28] G. Compere, S. de Buyl, E. Jamsin and A. Virmani, $G_{2}$ Dualities in D $=5$ Supergravity and Black Strings, Class. Quant. Grav. 26 (2009) 125016 [arXiv:0903.1645] [INSPIRE].

[29] E. Bergshoeff, W. Chemissany, A. Ploegh, M. Trigiante and T. Van Riet, Generating Geodesic Flows and Supergravity Solutions, Nucl. Phys. B 812 (2009) 343 [arXiv:0806.2310] [INSPIRE].

[30] L. Borsten, M.J. Duff, S. Ferrara, A. Marrani and W. Rubens, Small Orbits, Phys. Rev. D 85 (2012) 086002 [arXiv:1108.0424] [INSPIRE]. 
[31] S. Cecotti, S. Ferrara and L. Girardello, Geometry of Type II Superstrings and the Moduli of Superconformal Field Theories, Int. J. Mod. Phys. A 4 (1989) 2475 [inSPIRE].

[32] E. Cremmer and A. Van Proeyen, Classification of Kähler manifolds in $N=2$ vector multiplet supergravity couplings, Class. Quant. Grav. 2 (1985) 445 [INSPIRE].

[33] J. Luciani, Coupling of $O(2)$ Supergravity with Several Vector Multiplets, Nucl. Phys. B 132 (1978) 325 [inSPIRE].

[34] J. Bagger and E. Witten, Matter Couplings in $N=2$ Supergravity, Nucl. Phys. B 222 (1983) 1 [INSPIRE].

[35] A. Salam and E. Sezgin, Anomaly freedom in chiral supergravities, Phys. Scripta 32 (1985) 283.

[36] S. Randjbar-Daemi, A. Salam, E. Sezgin and J. Strathdee, An Anomaly Free Model in Six-Dimensions, Phys. Lett. B 151 (1985) 351 [inSPIRE].

[37] S. Ferrara, F. Riccioni and A. Sagnotti, Tensor and vector multiplets in six-dimensional supergravity, Nucl. Phys. B 519 (1998) 115 [hep-th/9711059] [INSPIRE].

[38] F. Riccioni and A. Sagnotti, Consistent and covariant anomalies in six-dimensional supergravity, Phys. Lett. B 436 (1998) 298 [hep-th/9806129] [INSPIRE].

[39] H. Nishino and E. Sezgin, New couplings of six-dimensional supergravity, Nucl. Phys. B 505 (1997) 497 [hep-th/9703075] [INSPIRE].

[40] M. Günaydin, H. Samtleben and E. Sezgin, On the Magical Supergravities in Six Dimensions, Nucl. Phys. B 848 (2011) 62 [arXiv: 1012.1818] [INSPIRE].

[41] D.V. Alekseevskii, Classification of quaternionic spaces with a transitive solvable group of motions, Math. USSR Izvestija 9 (1975) 297.

[42] E.B. Dynkin, Semisimple Subalgebras of Semisimple Lie Algebras, American Mathematical Society Translations, Series 26 (1957) 111.

[43] E.B. Dynkin, The Maximal Subgroups of the Classical Groups, American Mathematical Society Translations, Series 26 (1957) 245.

[44] A. Minchenko, The Semisimple Subalgebras of Exceptional Lie Algebras, Trans. Moscow Math. Soc. 67 (2006) 225.

[45] H. Lü, C. Pope and K. Stelle, Weyl group invariance and p-brane multiplets, Nucl. Phys. B 476 (1996) 89 [hep-th/9602140] [INSPIRE].

[46] L. Andrianopoli, R. D'Auria, S. Ferrara, P. Fré and M. Trigiante, RR scalars, U duality and solvable Lie algebras, Nucl. Phys. B 496 (1997) 617 [hep-th/9611014] [INSPIRE].

[47] L. Andrianopoli, R. D'Auria, S. Ferrara, P. Fré, R. Minasian and M. Trigiante, Solvable Lie algebras in type IIA, type IIB and M theories, Nucl. Phys. B 493 (1997) 249 [hep-th/9612202] [INSPIRE].

[48] E. Cremmer, B. Julia, H. Lü and C. Pope, Dualization of dualities. 1., Nucl. Phys. B 523 (1998) 73 [hep-th/9710119] [INSPIRE].

[49] A. Kleinschmidt and H. Nicolai, $E_{10}$ and $S O(9,9)$ invariant supergravity, JHEP 07 (2004) 041 [hep-th/0407101] [INSPIRE].

[50] E.A. Bergshoeff, M. de Roo, S.F. Kerstan, T. Ortín and F. Riccioni, IIA ten-forms and the gauge algebras of maximal supergravity theories, JHEP 07 (2006) 018 [hep-th/0602280] [INSPIRE]. 